\title{
Crustal thickness from seismic noise correlations in preparation for the InSight mission to Mars
}

\author{
Dissertation \\ zur Erlangung des mathematisch-naturwissenschaftlichen Doktorgrades \\ "Doctor rerum naturalium" \\ der Georg-August-Universität Göttingen \\ im Promotionsprogramm PROPHYS \\ der Georg-August University School of Science (GAUSS)
}

vorgelegt von

\section{Gesa Karen Becker}

aus Langenhagen

Göttingen, 2018 
Betreuungsausschuss

\section{Prof. Dr. Laurent Gizon}

Institut für Astrophysik, Georg-August-Universität Göttingen

Max-Planck-Institut für Sonnensystemforschung, Göttingen

Prof. Dr. Ulrich Christensen

Max-Planck-Institut für Sonnensystemforschung, Göttingen

Dr. Brigitte Knapmeyer-Endrun

Max-Planck-Institut für Sonnensystemforschung, Göttingen

Mitglieder der Prüfungskommision

Referent: Prof. Dr. Ulrich Christensen

Max-Planck-Institut für Sonnensystemforschung, Göttingen

Korreferent: Prof. Dr. Laurent Gizon

Institut für Astrophysik, Georg-August-Universität Göttingen

Max-Planck-Institut für Sonnensystemforschung, Göttingen

Weitere Mitglieder der Prüfungskommission:

PD Dr. Walter Goetz

Fakultät für Geowissenschaften und Geographie, Georg-August-Universität, Göttingen Max-Planck-Institut für Sonnensystemforschung, Göttingen

Dr. Brigitte Knapmeyer-Endrun

Max-Planck-Institut für Sonnensystemforschung, Göttingen

Prof. Dr. Wolfram Kollatschny

Institut für Astrophysik, Georg-August-Universität Göttingen

Prof. Dr. Andreas Tilgner

Institut für Geophysik, Georg-August-Universität, Göttingen

Tag der mündlichen Prüfung: 05.06.2018 


\section{Bibliografische Information der Deutschen Nationalbibliothek}

Die Deutsche Nationalbibliothek verzeichnet diese Publikation in der Deutschen Nationalbibliografie; detaillierte bibliografische Daten sind im Internet über http: //dnb . d-nb. de abrufbar.

Cover: 'Mars Colorized Viking Mosaic' and 'The Blue Marble: Land Surface, Ocean Color and Sea Ice' courtesy of NASA.

Background image and cover design provided by Felix Mackebrandt.

ISBN 978-3-944072-64-7

uni-edition $\mathrm{GmbH} 2018$

http://www.uni-edition.de

(c) Gesa Karen Becker

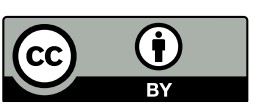

This work is distributed under a

Creative Commons Attribution 3.0 License

Printed in Germany 



\section{Contents}

$\begin{array}{ll}\text { Zusammenfassung } & 6\end{array}$

Summary 6

1 Introduction $\quad 11$

$1.1 \quad$ Mars ................................. 11

1.2 InSight mission . . . . . . . . . . . . . . . . . . . . . . . 14

1.3 Seismicity on Mars . . . . . . . . . . . . . . . . . . . . 18

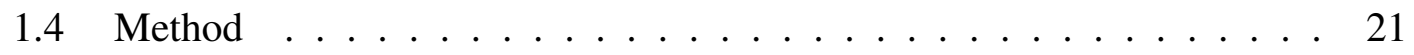

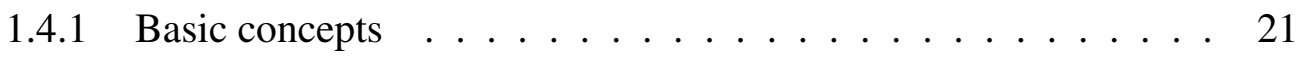

1.4.2 Passive seismic interferometry . . . . . . . . . . . 23

1.5 Applications of passive seismic interferometry . . . . . . . . . . . 27

2 Crustal thickness from vertical component ambient seismic noise data 31

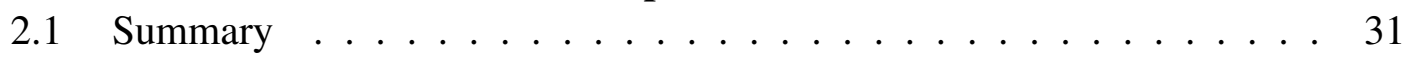

2.2 Introduction . . . . . . . . . . . . . . . . 32

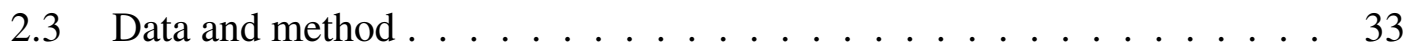

$2.3 .1 \quad$ Data . . . . . . . . . . . . . . . 33

2.3 .2 Method ....................... 34

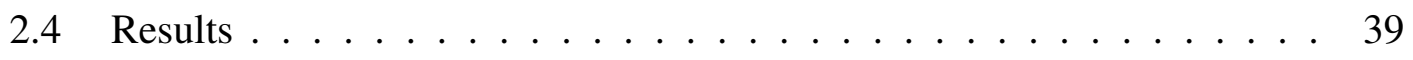

2.4.1 Spatial consistency . . . . . . . . . . . . . . 39

2.4.2 Temporal properties and consistency . . . . . . . . . . . 40

2.4 .3 Moho depths . . . . . . . . . . . . . . . . 43

2.4 .4 Profile P4 . . . . . . . . . . . . . . . . . 46

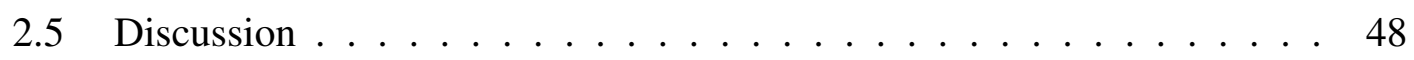

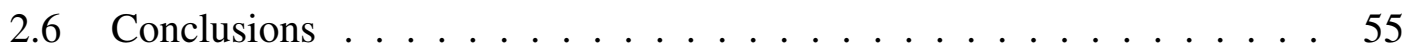

A2.A Appendix . . . . . . . . . . . . . . . . . . 57

3 Crustal thickness from horizontal component auto- and cross-correlations 61

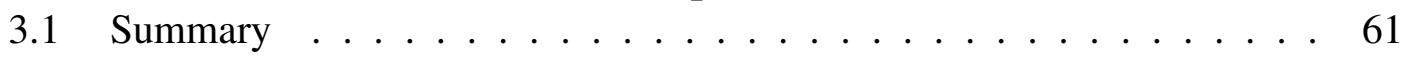

3.2 Introduction . . . . . . . . . . . . . . . . . 62

3.3 Data and study area . . . . . . . . . . . . 63

3.4 Method .......................... 64

3.4.1 Processing and calculation of auto- and cross-correlations . . . . 64

3.4.2 Determination of $v_{\mathrm{P}} / v_{\mathrm{S}}$-ratio and crustal thickness . . . . . . 65

3.5 Results ........................... 67

3.5 .1 Temporal properties . . . . . . . . . . . . . 67 
3.5.2 Spatial consistency . . . . . . . . . . . . . . 68

3.5.3 Consistency across correlations . . . . . . . . . . . 70

3.5.3.1 Vertical-horizontal component cross-correlations . . . 70

3.5.3.2 Horizontal component auto- and cross-correlations . . . 71

3.5.3.3 Vertical component autocorrelations . . . . . . . . 72

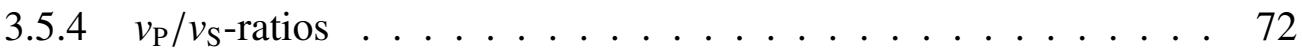

3.5.5 Moho depths . . . . . . . . . . . . . . . 74

3.6 Discussion . . . . . . . . . . . . . . . 75

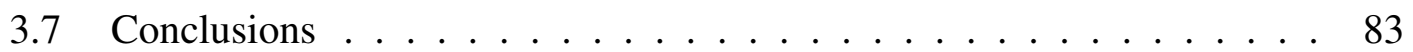

4 Discussion and Outlook $\quad 85$

5 Conclusions $\quad 91$

$\begin{array}{ll}\text { Bibliography } & 93\end{array}$

$\begin{array}{ll}\text { Acknowledgements } & 107\end{array}$

$\begin{array}{ll}\text { Curriculum vitae } & 109\end{array}$ 


\section{Zusammenfassung}

Die Entstehung terrestrischer Planeten wirft noch viele Fragen auf. Die Untersuchung des Inneren des Planeten Mars mit Hilfe seismischer Wellen kann zur Beantwortung dieser Fragen beitragen. Große Teile der Marskruste sind aus der Frühzeit der Entwicklung des Planeten erhalten, da Mars keine Plattentektonik aufweist. Informationen über die Mächtigkeit und den Aufbau der Kruste können somit zu unserem Verständnis der Entstehung und Entwicklung terrestrischer Planeten beitragen.

Die InSight Mission zum Mars wird ein Seismometer auf der Oberfläche des Planeten absetzen. Um die seismischen Daten bestmöglich auszunutzen, wird in dieser Arbeit eine Methode entwickelt und vorgestellt, die aus seismischem Rauschen die Reflektivität des Untergrundes abbildet und die Dicke der Kruste bestimmt. Die hier entwickelte Methode macht von 'passiver seismischer Interferometrie' Gebrauch. Durch die Berechnung von Auto- und Kreuzkorrelationen erhält man die Reflektionsantwort des Untergrundes. Anschließend wird die Methode mit Hilfe von Daten getestet, die an seismischen Stationen in Zentral- und Osteuropa aufgenommen wurden. Diese Stationen befinden sich in Gebieten, wo die Erdkurste unterschiedliche Mächtigkeiten aufweist, welche vergleichbar sind mit Abschätzungen für die InSight Landestelle.

Die Analyse der Daten besteht aus Glätten des Spektrums, Auto- oder Kreuzkorrelation der Phase, im Zeit-Frequenz-Bereich nach Phase gewichtetes Stapeln und Bandpassfiltern (hauptsächlich im Bereich 1-2 Hz). Diese Schritte lassen sich erfolgreich auf Daten von Horizontal- und Vertikalkomponenten anwenden. Die Daten der Vertikalkomponenten resultieren in jeweils ein Autokorrelationsergebnis für die einzelnen Messstationen. Die Daten der Horizontalkomponenten ergeben zwei Autokorrelationen, in Nord-Südund Ost-West-Richtung, sowie zwei Kreuzkorrelationen von gemischter Richtung. Die berechneten Korrelationen sind zeitlich und räumlich stabil, aber es lässt sich für alle Komponenten ein klarer Zusammenhang mit anthropogen erzeugtem Rauschen erkennen. Die Korrelationen, die für die Vertikalkomponenten berechnet werden, konvergieren nach 6 Monaten zu einem stabilen Ergebnis. Die Korrelationen der horizontalen Komponenten benötigen dafür mehr Daten. Dies gilt insbesondere für die Kreuzkorrelationen. Aus Informationen über seismische Geschwindigkeiten und geschätzte Moho-Tiefen wird ein Unsicherheits-Ziel-Fenster berechnet. In diesem Fenster wird eine Reflektivitätsänderung erwartet. Im Fall der Vertikalkomponenten wird die Reflektivitätsänderung, welche im Zusammenhang mit der Moho steht, automatisch innerhalb des Ziel-Fensters bestimmt. Für die Horizontalkomponenten muss dieser Vorgang manuell durchgeführt werden, um alle vier Korrelationsergebnisse zu berücksichtigen. Die bestimmten Laufzeiten werden mit Hilfe der a priori seismischen Geschwindigkeiten in Tiefe umgerechnet.

Die berechneten Moho-Tiefen stimmen mit Ergebnissen anderer Studien überein und ergeben konsistente Resultate für die Daten von Horizontal- und Vertikalkomponenten. 
Außerdem können weitere Reflektoren bei größeren und niedrigeren Tiefen identifiziert werden. Durch Kombination der bestimmten Laufzeiten der Korrelationen von Vertikalund Horizontalkomponenten lassen sich Abschätzungen des $v_{\mathrm{P}} / v_{\mathrm{S}}$-Verhältnisses erhalten. Jedoch sind diese Verhältnisse sehr variabel und ergeben teilweise unrealistische Werte. Diese Variabilität lässt sich zum Beispiel durch nicht-vertikale Einfallswinkel und daraus resultierende abweichende Laufwege der seismischen Wellen erklären. Aus diesem Grund müssen sie mit Vorsicht gehandhabt werden. Trotzdem können die Autokorrelationen der Horizontalkomponenten zusätzlich genutzt werden um mögliche krustale Anisotropie unterhalb der Messstationen zu detektieren.

Zusammenfassend lässt sich sagen, dass die Krustenmächtigkeit aus seismischem Rauschen für eine einzelne Station extrahiert werden kann, für verschiedene Seismometerarten, wenn nur geringe Mengen an Daten vorhanden sind, ohne starke seismische Quellen und unabhängig vom vorliegenden Untergrund. Dies zeigt das Potential der vorgestellten Methode im Hinblick auf die InSight Mission. 


\section{Summary}

The formation of terrestrial planets is still poorly understood. Studying the interior of Mars by analysing seismic waves can help to answer questions about that process. In the absence of plate tectonics, Mars has retained much of its crust from early stages of the planet's evolution. Knowledge of its crustal thickness and structure can therefore contribute to the understanding of terrestrial planet formation and evolution.

In preparation for the InSight seismometer installation on Mars, a method is developed to image the reflectivity of the subsurface and extract the crustal thickness from ambient seismic noise. The developed method makes use of passive seismic interferometry by calculating auto- and single-station cross-correlations to obtain the reflection response of the subsurface. It is then tested on seismic stations across Central and Eastern Europe with varying crustal thicknesses, comparable to estimates for the InSight landing site.

A processing scheme of spectral smoothing, phase auto- or cross-correlation, timefrequency domain-phase weighted stacking and bandpass filtering, predominantly between 1-2 Hz, is introduced. This processing scheme works well for the vertical and horizontal component data. One autocorrelation result is obtained for each station from the vertical component data. The horizontal component data gives two autocorrelation results, in the North-South and East-West directions, and two cross-correlation results of mixed directions. The obtained correlations are spatially and temporarily stable, but for all components a correlation with the presence of cultural noise can be observed. The vertical component correlations converge to a stable result after $\sim 6$ months of data, whereas the horizontal component correlations require more data, especially the cross-correlations. Prior information, in the form of seismic velocity and the predicted Moho depth, are included as an uncertainty target window of expected reflectivity change. The reflectivity change associated with the Moho is automatically determined inside the uncertainty window in case of the vertical component autocorrelations. For the horizontal component correlations, this needs to be done manually to include all four correlation results. The determined lag times are converted to depths using the prior information about the seismic velocities.

The obtained Moho depths compare well with results obtained from other studies using different methods and are consistent across vertical and horizontal component correlations. Furthermore, it is possible to identify additional deeper and shallower reflectors. The combination of the determined lag times of Moho related reflectivity change from the horizontal and vertical component correlations gives the possibility to obtain estimates of the $v_{\mathrm{P}} / v_{\mathrm{S}}$-ratios. However, these ratios show large variability and sometimes unreasonable values and therefore need to be treated with caution. This variability can be explained by e.g., non-vertical incidence, and the resulting different paths of the waves. The horizontal component autocorrelations can nevertheless be used to detect possible crustal anisotropy 
below the station.

In general, crustal thickness can be extracted from seismic noise correlations for a single station, for different seismometer types, when only moderate amounts of data are available, without the need for strong seismic sources, and independent of the structure of the subsurface. All this makes the introduced method a promising tool for the InSight mission. 


\section{Introduction}

The most difficult thing is the decision to act, the rest is merely tenacity.

Amelia Earhart

In 2018, the InSight mission will place a single seismic station on Mars. This provides new exciting opportunities to extend single-station methods and in the end learn more about terrestrial planet formation. For the latter, it is necessary to make the most out of the one station at hand and use all data available. Here, auto- and single-station cross-correlation methods are used to extract the crustal thickness from seismic noise on Earth in preparation for the data return from Mars. This chapter will provide background information on the planet Mars and its expected seismicity, introduce the InSight mission and its goals, and explain the underlying principles and the method of seismic interferometry used throughout this work. In addition, it will give a brief overview of where this method has been successfully applied before.

\subsection{Mars}

The contents of this section were compiled from Barlow (2008) and Taylor (2010) unless otherwise indicated.

Mars has always been a fascinating object and a focus of scientific research. An image of Mars can be seen in Fig. 1.1. First observations were made from Earth with the naked eye and followed later on by the use of telescopes. For the last 50 years it has been possible to learn more about Mars from close up using space-based instruments. Mars is a neighbouring planet of the Earth and the fourth planet from the Sun. An overview of its orbital and physical properties can be found in Table 1.1. The sidereal Martian day, named a 'Sol', is $24.659722 \mathrm{hr}$, compared to the Earths $23.93447 \mathrm{hr}$ rotation period. Mars is approximately half the radius of Earth with roughly 38 \% of Earth's gravity. It is surrounded by a thin atmosphere with an average pressure of $600 \mathrm{~Pa}$ at the surface.

Two small moons, Phobos and Deimos, which can be seen in Fig. 1.1, right side, accompany Mars. Phobos is the innermost of the two moons with a semi-major axis around Mars of $9378.5 \mathrm{~km}$ and an orbital period of 0.31891 days. The shape of the moon is highly irregular and its mean radius is $11.2667 \mathrm{~km}$. Deimos is further away from Mars and smaller, with a semi-major axis of $23458.8 \mathrm{~km}$ and a mean radius of $6.2 \mathrm{~km}$. Its orbital period of $1.26244 \mathrm{~d}$ means that it is roughly in synchronous rotation with Mars. 


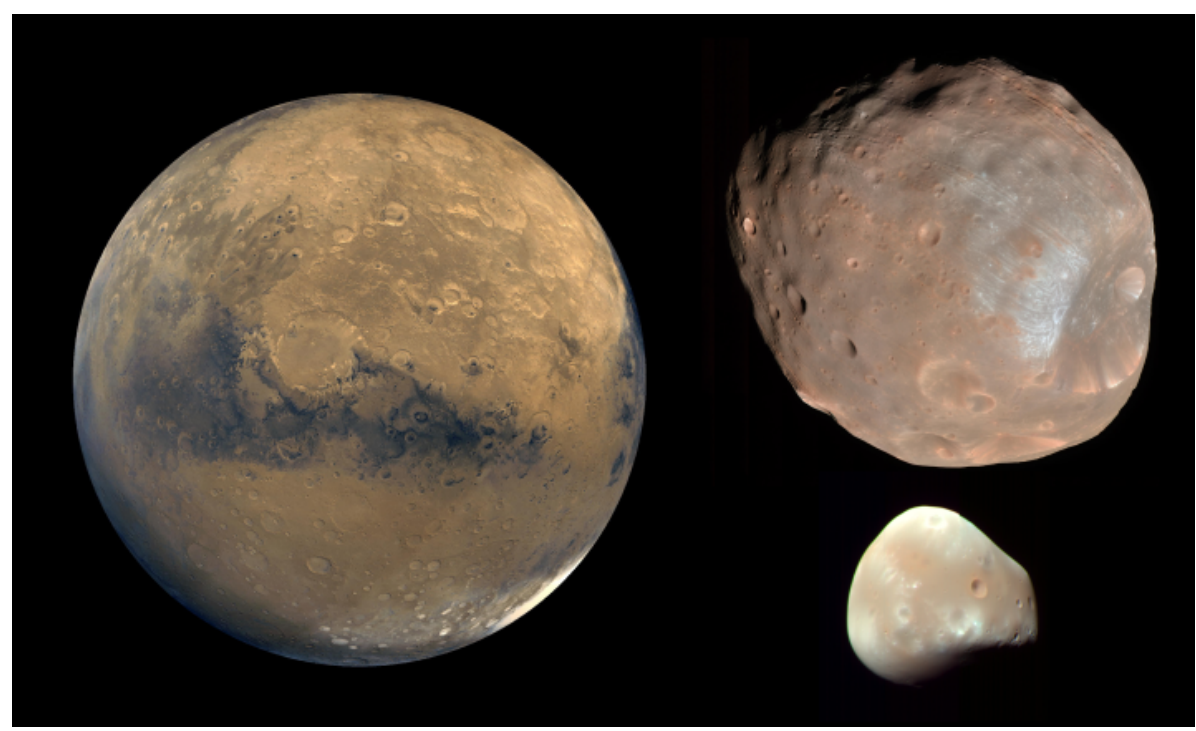

Figure 1.1: Left: Mosaic image of Mars composed of 100 Viking Orbiter images taken in 1980. Right: HiRISE images taken from Mars Reconnaissance Orbiter Phobos (top) and Deimos (bottom). Images not to scale. Courtesy of NASA/JPL-Caltech/USGS/University of Arizona.

Table 1.1: Orbital and physical properties of Mars

\begin{tabular}{ll}
\hline Mass & $6.4185 \times 10^{23} \mathrm{~kg}$ \\
Volume & $1.6318 \times 10^{11} \mathrm{~km}^{3}$ \\
Mean density & $3993 \mathrm{~kg} \mathrm{~m}^{-3}$ \\
Mean radius & $3389.508 \mathrm{~km}$ \\
Surface gravity & $3.71 \mathrm{~m} \mathrm{~s}^{-2}$ \\
Solar day & $24.659722 \mathrm{hr}$ \\
Sidereal rotation period & $24.622958 \mathrm{hr}$ \\
Sidereal orbital period & $686.98 \mathrm{~d}$ \\
Semi-major axis & $2.2792 \times 10^{8} \mathrm{~km}$ \\
Inclination & $1.84969142^{\circ}$ \\
Eccentricity & 0.0933941 \\
\hline
\end{tabular}

Phobos and to a lesser extend Deimos exert tidal stresses on Mars. Measurements of the tide could be used to constrain the size of the Martian core (Lognonné \& Mosser 1993, Van Hoolst et al. 2003).

The topography of Mars has been determined by the Mars Orbiter Laser Altimeter (MOLA), providing a vertical accuracy of up to $\sim 1 \mathrm{~m}$. One of the main features is the dichotomy in elevation between the elevated southern highlands and the lower northern plains (Fig. 1.4). Other important features are the Tharsis volcanic province with high elevations near the equator at $100^{\circ} \mathrm{W}$, the giant impact basins Hellas $\left(40^{\circ} \mathrm{S}, 65^{\circ} \mathrm{W}\right)$, Argyre $\left(50^{\circ} \mathrm{S}, 40^{\circ} \mathrm{W}\right)$, and Isidis $\left(15^{\circ} \mathrm{N}, 85^{\circ} \mathrm{E}\right)$, the Elysium volcanic province $\left(25^{\circ} \mathrm{N}\right.$, $145^{\circ} \mathrm{E}$ ) and the rift valley Valles Marineris, stretching between $30^{\circ} \mathrm{W}$ and $110^{\circ} \mathrm{W}$ along the equator (Wieczorek 2015). The highest point on the surface of Mars is the Olympus Mons volcanic summit with a height of $21.287 \mathrm{~km}$. The lowest point is the Hellas impact basin with $-8.180 \mathrm{~km}$. Zero elevation corresponds to the Martian geoid, with the geoid 


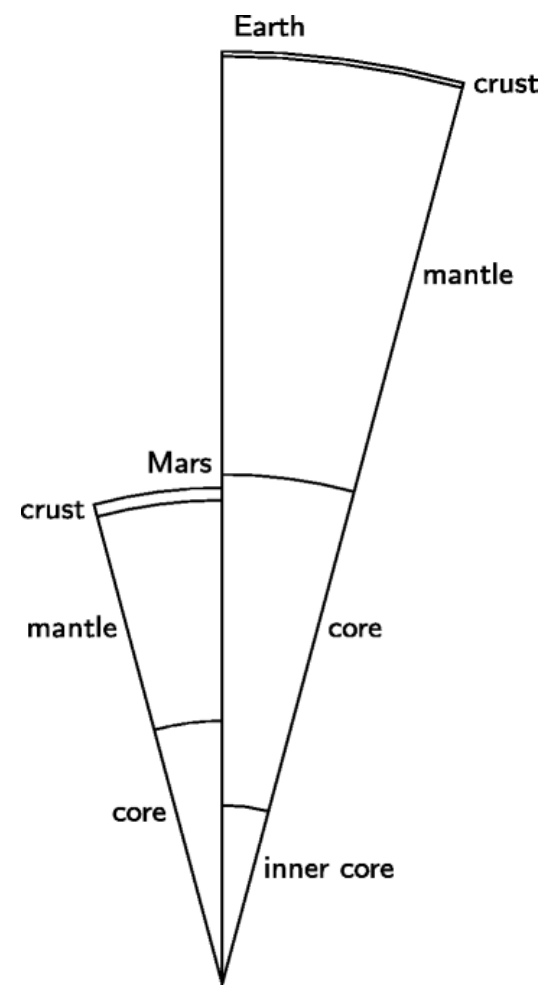

Figure 1.2: Sketch of the internal structure of Mars with respect to Earth (taken from Helffrich (2017)).

here referring to the equipotential surface of Mars, with a distance of the planet's mean radius from its centre of mass at the equator (Neumann et al. 2004).

Measurements of the Martian gravity field, for example, from Doppler tracking data by Mars Global Surveyor, Mars Odyssey and Mars Reconnaissance Orbiter, put constraints on the planetary interior structure. Information about the internal distribution of mass and the tidal Love number $k_{2}$ can be deducted from the measurements (e.g. Konopliv et al. 2016). The principal moment of inertia of Mars has been derived from the zonal harmonics of the Doppler measurements and the precession of the rotation axis (e.g. Kaula 1979, Folkner et al. 1997). The mass or mean density together with the tidal Love number $k_{2}$ and the moment of inertia and assumption on the bulk chemistry, for example, from meteorites, provide the basis for estimate of the internal structure of Mars (Panning et al. 2017a). It was concluded that Mars consists of a crust, a mantle and a core, very similar to Earth. The crust is thicker in the southern hemisphere than in the northern hemisphere. Wieczorek \& Zuber (2004) estimate the mean crustal thickness to vary between 33 and $81 \mathrm{~km}$ (see below). The adjoining mantle extends down to $\sim 1700-2100 \mathrm{~km}$. The core is iron-rich and has a radius of $\sim 1300-1700 \mathrm{~km}$. A general overview of the structure of Mars, in comparison with Earth can be found in Fig. 1.2.

It was shown by spacecraft exploration (e.g. Mars Global Surveyor), that Mars does not possess a global magnetic field, but localized traces of fields frozen into the crust could be detected. This indicates that at some point during its evolution, Mars had a strong magnetic field, which magnetized rocks in the crust.

By analysing gravity together with topography measurements, correlations between gravity and topography can be observed. Crustal thickness variations can be modelled 
by assuming density variations in crust and mantle. A summary of possible methods to extract crustal thickness from gravity and topography data can be found in Wieczorek \& Zuber (2004). A simple explanation of the gravity field is, that it is a consequence of the surface topography and topography of the Moho. This approach was used, for example, by Zuber et al. (2000), who assumed a uniform crustal density and a minimum crustal thickness at a specific location to calculate global variations in crustal thickness. Instead of providing a minimum crustal thickness, the inversion could also be linked to a thickness estimate at a specific location, a 'tie-point.' An important parameter to determine the crustal thickness is the assumed crustal density (Goossens et al. 2017). Wieczorek $\&$ Zuber (2004) assumed a rather conservative range of average crustal density of 2700$3100 \mathrm{~kg} \mathrm{~m}^{-3}$, which resulted into an estimate of average crustal thickness of $57 \pm 24 \mathrm{~km}$. Neumann et al. (2004) also used an average crustal density of $2900 \mathrm{~kg} \mathrm{~m}^{-3}$ to determine the crustal structure. They came to the conclusion that an average crustal thickness of $>45 \mathrm{~km}$ is consistent with the geology and thermal history of Mars. In addition, they detected a distinction between the northern and southern hemispheres, with average crustal thicknesses of $32 \mathrm{~km}$ and $58 \mathrm{~km}$, respectively. The results obtained by Neumann et al. (2004) and Wieczorek \& Zuber (2004) agree well with each other. Goossens et al. (2017) further constrained the average crustal density to $2582 \pm 209 \mathrm{~kg} \mathrm{~m}^{-3}$, which is lower than previously assumed and may lead to a different average crustal thickness. Plesa et al. (2016) used average crustal thickness values between $29.5 \mathrm{~km}$ to $87.1 \mathrm{~km}$ to estimate the heat flux at the InSight landing site. An example of two global Martian crustal thickness maps for an average crustal density of $2900 \mathrm{~kg} \mathrm{~m}^{-3}$, assuming different minimum crustal thickness values can be found in Fig. 1.3. Overall there is an uncertainty of a factor 2-3 in crustal thickness (Wieczorek \& Zuber 2004). As will be explained in section 1.2, one of the goals of the SEIS instrument on board the InSight mission is to provide such a tie-point of crustal thickness to distinguish between crustal models and reduce this uncertainty. The goal of this work is to provide a method for determining this thickness from single-station data.

All these measurements provide constraints on the interior structure, but only analysis of seismic propagation can provide further details. Traces of volcanic and tectonic activity provide evidence that Mars has been seismically active. Analysis of cooling rates and mapping of tectonic faults lead to the conclusion that Mars remains seismically active today (see section 1.3). This possible seismic activity, along with seismic noise and meteorite impacts, can be used to study the interior by placing a seismic station on the planet.

\subsection{InSight mission}

To date, seven space missions have successfully landed on Mars, with Viking $1 \& 2$ being the first to do so in 1976 (Klein et al. 1976). The latest mission to reach the surface and return scientific data is the Mars Science laboratory mission with the Curiosity rover, launched in 2011 (Grotzinger et al. 2012). An overview of successful Mars missions can be found in Tab. 1.2 with their respective landing sites in Fig. 1.4 (NASA's Mars Exploration program website). Three missions are scheduled to launch and land on the Martian surface in the near future. ESA's ExoMars mission Part 2, and NASA's Mars 2020 
(a)

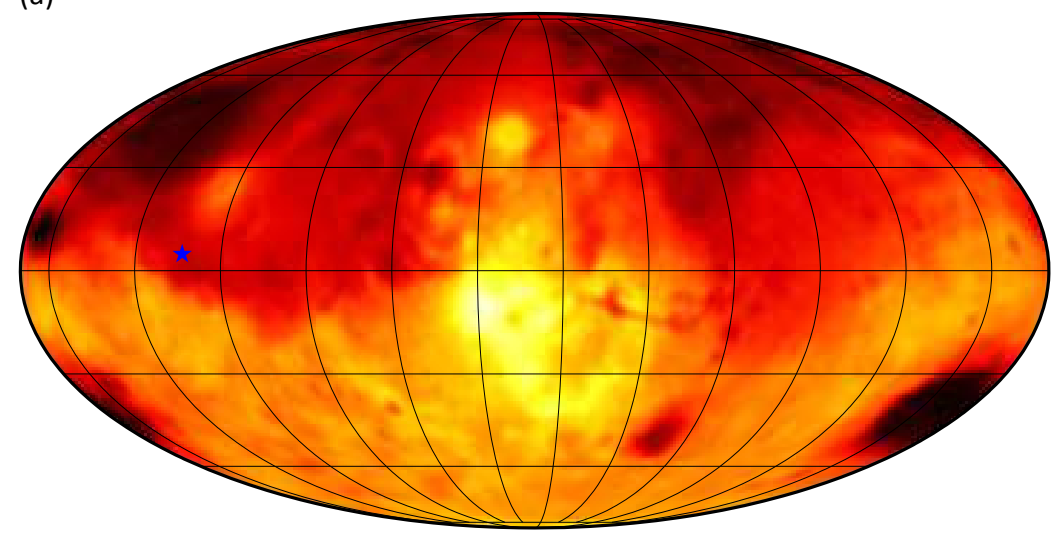

(b)

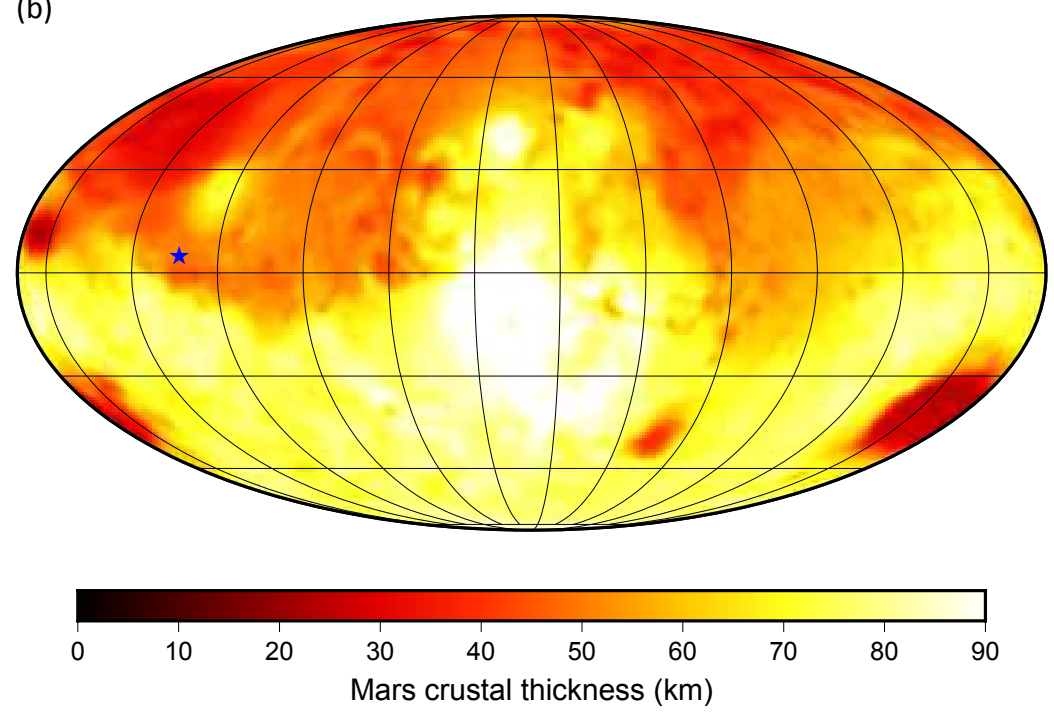

Figure 1.3: Comparison of two Martian crustal thickness estimates for an average crustal density of $2900 \mathrm{~kg} \mathrm{~m}^{-3}$ after Plesa et al. (2016). Blue star indicates InSight landing site (Golombek et al. 2017). (a) Minimum crustal thickness of $1 \mathrm{~km}$, resulting in an average crustal thickness of $40 \mathrm{~km}$. (b) Minimum crustal thickness of $20 \mathrm{~km}$, resulting in an average crustal thickness of $63.3 \mathrm{~km}$.

Table 1.2: Past and present successful surface Mars missions compiled from NASA's Mars Exploration program website and Taylor (2010).

\begin{tabular}{lcl}
\hline Mission & Year & Period of operation \\
\hline Viking 1 & 1975 & 2245 sols \\
Viking 2 & 1975 & 1281 sols \\
Mars Pathfinder/Sojourner & 1996 & 83 sols \\
Mars Exploration Rover Spirit & 2003 & 2208 sols \\
Mars Exploration Rover Opportunity & 2003 & operational \\
Phoenix & 2007 & 157 sols \\
Mars Science Laboratory Curiosity & 2011 & operational \\
\hline
\end{tabular}


mission are scheduled for launch in 2020 and will provide new insights on the possibility of life on Mars. Before that, the InSight mission is scheduled for launch in 2018 and will be introduced in the following.

The InSight Mission (Interior Exploration using Seismic Investigations, Geodesy and Heat Transport) to Mars is part of NASA's discovery program. Its goal is to place a geophysical lander on the surface of Mars (Banerdt et al. 2013), and it aims to help in the understanding of terrestrial planet formation. Mars is an excellent candidate for this. It has undergone all the major early processes shaping the terrestrial bodies (e.g. internal heating and differentiation), but has retained more complete traces of these processes than Earth. In the absence of plate tectonics, the majority of the Martian crust has been preserved from the early development of the planet and can therefore help in the understanding of the formation of terrestrial planets.

Six science investigations will be conducted:

- Determination of the size, composition and physical state of the core.

- Determination of the thickness and structure of the mantle.

- Determination of the thickness and structure of the crust.

- Determination of the thermal state of the planet's interior.

- Measurement of the rate and geographical distribution of Mars' internal seismic activity.

- Measurement of the impact rate of meteorites on the surface.

The launch of the mission is scheduled for May 5, 2018 with landing planned for November 26, 2018 at western Elysium Planitia (Golombek et al. 2017). Fig. 1.4 shows the planned landing site at about $4.5^{\circ} \mathrm{N}$ and $135.9^{\circ} \mathrm{E}$, along with locations of other Mars missions. Surface operations will run for roughly $728 \mathrm{~d}$ and provide approximately $29 \mathrm{~GB}$ of data during one Martian year.

In order to achieve the scientific goals, the lander will carry three different instruments:

$\mathbf{H P}^{3}$ (Heat flow and Physical Properties Probe) Will measure the temperature profile in the subsurface together with thermal conductivity measurements (Spohn et al. 2012).

RISE (Rotation and Interior Structure Experiment) Uses the lander's radio link to measure the rotation of Mars and variations in its rotation axis (Folkner et al. 2012).

SEIS (Seismic Experiment for Interior Structure) Will monitor seismic activity and measure seismic waves travelling through the planet (Lognonné et al. 2015).

$\mathrm{HP}^{3}$ and SEIS will be deployed to the surface by a robotic arm (Instrument deployment system, IDS), whereas RISE will be located on the lander. An overview of the setup of the InSight lander can be seen in Fig. 1.5.

The studies in the following chapters were conducted in preparation of the SEIS installation on Mars. SEIS consists of a three-component ultra-sensitive very broad-band seismometer (VBB) and a collocated miniature three-component short-period seismometer 


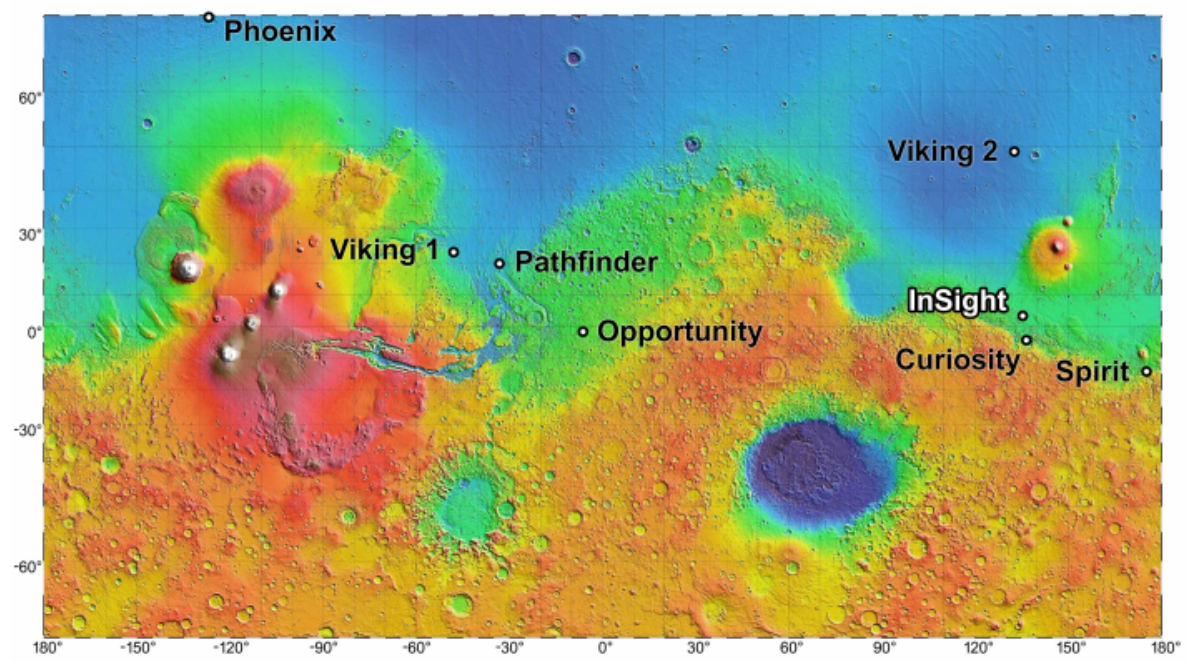

Figure 1.4: Topographic map of Mars constructed from Mars Orbiter Laser Altimeter data, indicating the planned landing site of the InSight mission, along with landing site of former missions. Lowest elevations in dark blue; highest elevations white. Elevation difference between green and orange approximately $4 \mathrm{~km}$. Courtesy of NASA/JPL-Caltech.

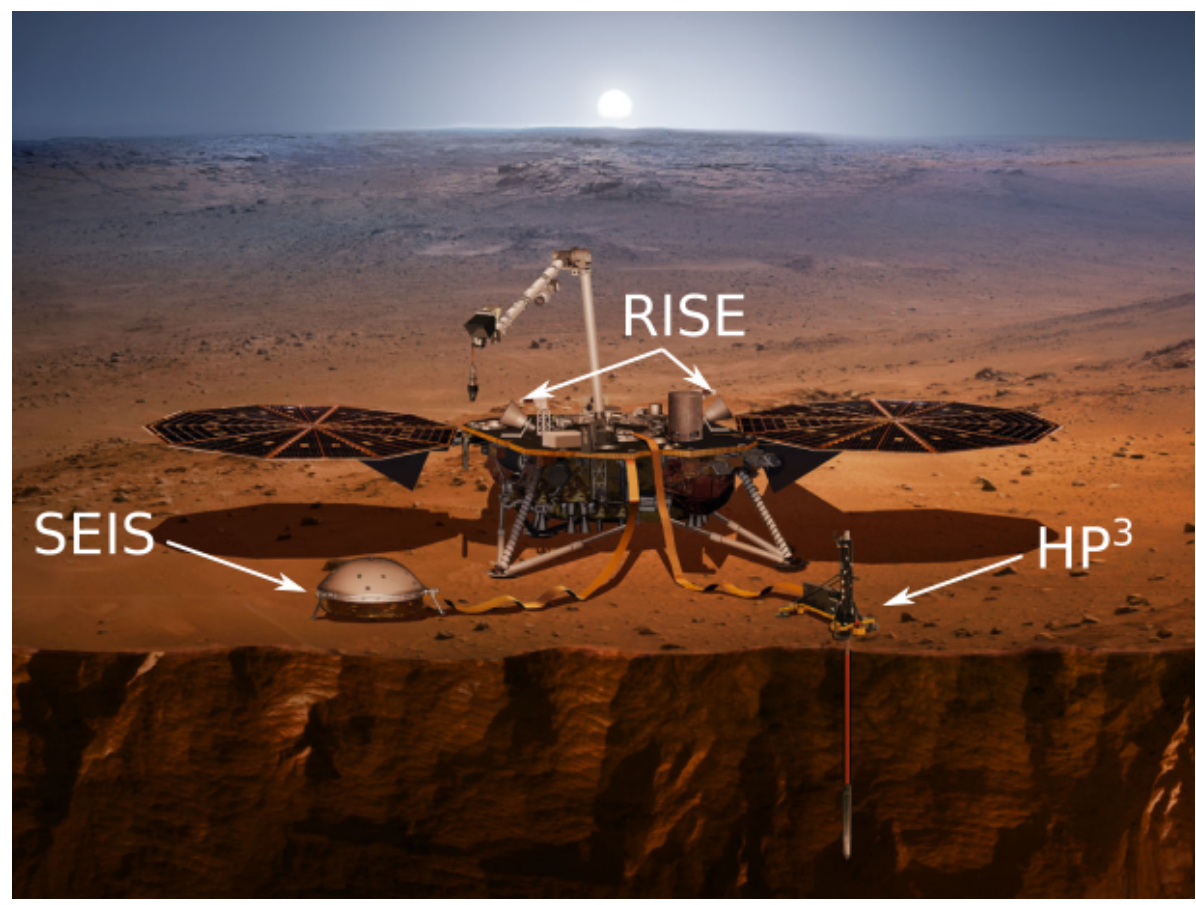

Figure 1.5: Artists view of the InSight lander on Mars, showing the SEIS experiment on the ground on the left and $\mathrm{HP}^{3}$ on the right. RISE is located on top of the lander. Courtesy of NASA/JPLCaltech. 
(SP). The short-period seismometer is designated to record frequencies between $1-50 \mathrm{~Hz}$, whereas the broad-band seismometer records frequencies between 0.01-1 Hz (Mimoun et al. 2017). The seismometers are mounted on a levelling-system in order to ensure horizontal orientation and will be protected from weather by a wind and thermal shield. The SP will be most sensitive to local and regional seismic events, with its peak sensitivity at higher frequencies (Teanby 2015). One of the goals of the SEIS experiment is to provide an absolute tie-point of crustal thickness. In combination with heat flow measurements, this gives the possibility to decide on a model of crustal formation.

InSight will be the first mission to aim for the surface of Mars after the successful Mars Science Laboratory - Curiosity in 2012 and the failed ESA ExoMars mission in 2016.

\subsection{Seismicity on Mars}

Observations of traces of volcanic and tectonic activity lead to the suggestion that Mars might have been tectonically active at some point during its evolution. Today, Mars no longer shows signs of plate tectonics, but is covered by a stagnant lid (single plate) (Barlow 2008). Without plate tectonics, which are an important source of seismicity on Earth, other sources of seismic signal need to be considered. Until the SEIS instrument successfully concludes its investigation of the seismic activity and meteorite impact rate, we rely on other methods to estimate the current seismic activity. The only in situ measurements of seismicity come from the Viking 2 seismometer in 1976 and 1977. In this case the seismometer was located on top of the lander and ground coupling was was given through the legs of the lander. The observations made from the Viking seismic experiment lead to the conclusions that the seismic background noise due to wind and pressure fluctuations is low. In addition, it was deduced, that Mars is probably less active than Earth, because no conclusive events were detected (Anderson et al. 1977). This lower seismic activity than Earth is also the result of other theoretical studies based on surface fault mapping (Golombek et al. 1992, Golombek 2002, Knapmeyer et al. 2006) or lithospheric thermoelastic cooling models (Phillips 1991). From these estimates it is concluded that potentially 1-10 events with a moment magnitude $M_{\mathrm{w}}>5.3$ may be observed throughout the mission duration (Panning et al. 2015) and many more events with a smaller moment magnitude. Knapmeyer et al. (2006) predict up to 572 events with $M_{\mathrm{w}}>4$ per year as the upper limit case. The studies using surface fault mapping to estimate the seismicity assume that those surface faults are still active today, which is however uncertain. Plesa et al. (2018) further extended the estimates by using 3-D numerical thermal evolution models and including convective stresses. The resulting annual seismic moment release of $5.7 \times 10^{16}-3.9 \times 10^{19} \mathrm{Nm}$ falls within the range predicted by Golombek (2002) and Knapmeyer et al. (2006). Fig. 1.6 shows the different size-frequency distributions obtained by the studies mentioned above. Overall, the Martian seismicity is believed to range between the intraplate seismicity of Earth and seismicity related to shallow quakes on the Moon. Taylor et al. (2013) estimated the seismic activity in the Cerberus Fossae region on Mars using fault mapping. The Cerberus Fossae region is located approximately $1500 \mathrm{~km}$ north-east of the InSight landing site. It is interpreted as a long graben system (Vetterlein \& Roberts 2010). There are indications of on-going activity in the form 


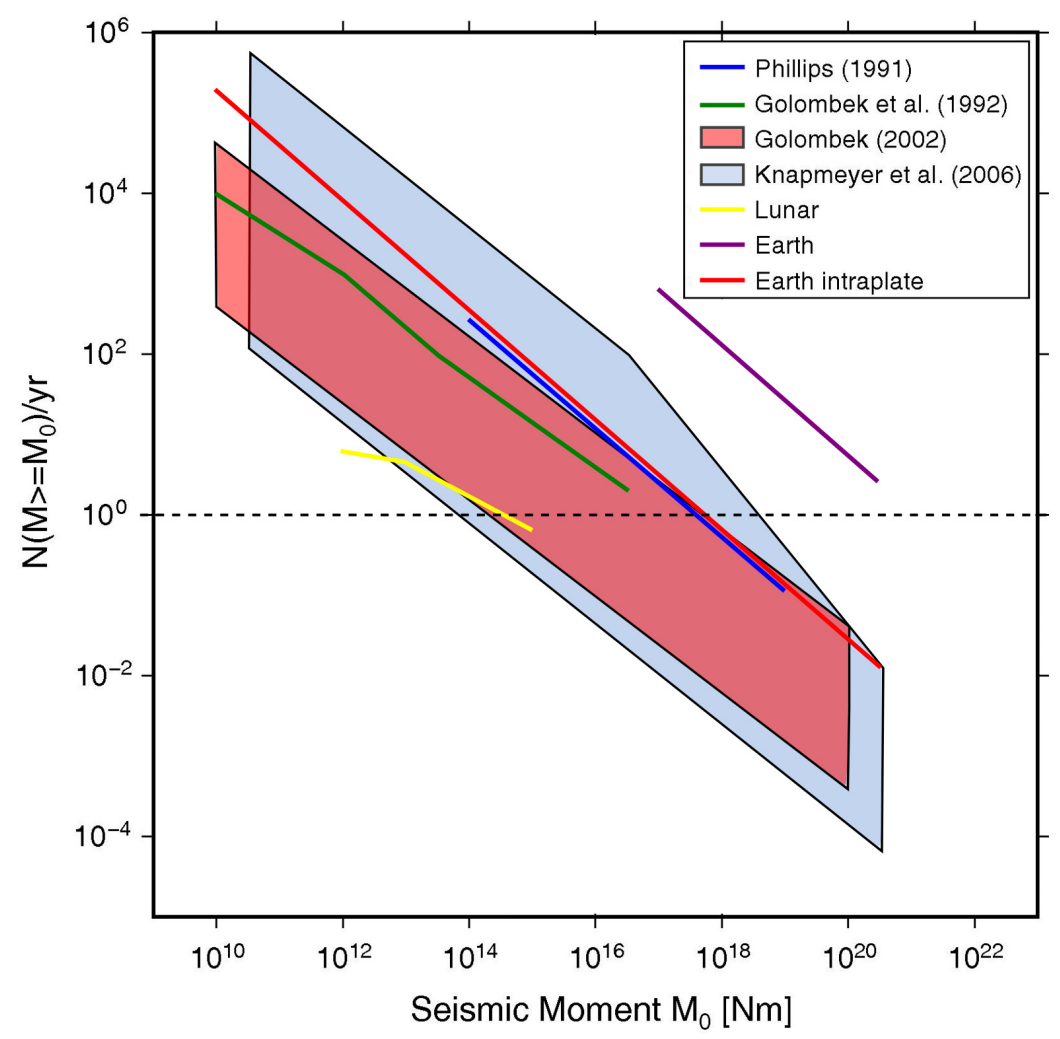

Figure 1.6: Comparison of the estimates of size-frequency distributions of seismic events obtained from different studies, as well as distributions for the Earth and Moon (adapted and compiled from Knapmeyer et al. (2006), Panning et al. (2015)). Dashed horizontal line indicates the level above which events would be expected to be seen during the InSight mission (Panning et al. 2015).

of fresh bolder trails originating from Marsquakes (Roberts et al. 2012). This region is therefore a probable source of seismic signal for the InSight mission. It was estimated that this area produces a seismic moment release of $1.14_{-0.62}^{+1.04} \times 10^{17} \mathrm{Nm} / \mathrm{yr}$. This falls into the range of the global annual moment and could be an indication that Mars is more seismically active than estimated by other studies. From the local annual seismic moment release, it was calculated that between $2.7 \times 10^{0}$ and $3.5 \times 10^{5}$ events in Cerberus Fossae would be detectable over the nominal InSight mission duration.

In order to determine the size of the core, seismic phases reflected at the core-mantle boundary or transmitted through the core are necessary. The amplitudes of these phases need to be higher than the background noise level (see below), in order to be detected. (Panning et al. 2017b) predict events with $M_{\mathrm{w}}>4.5\left(\sim 10^{16} \mathrm{Nm}\right)$ to be sufficient to create such amplitudes. Approximately ten such events are expected to be recorded during the mission duration.

Another source of seismic signals are meteorite impacts. It might be possible to observe craters created by such impacts using orbital images, thereby providing location and additional constraints. Teanby \& Wookey (2011) determined through modelling that a seismic moment of over $10^{13} \mathrm{Nm}\left(M_{\mathrm{w}}=2.6\right)$ is necessary to record and detect a teleseismic event under nominal noise conditions. This is equal to an impact producing a crater of at least $100 \mathrm{~m}$ diameter. Such an event is detectable about once every ten years under nominal conditions. Assuming more favourable low noise conditions, they predict that 
one event in teleseismic distances could be recorded per year. Teanby \& Wookey (2011) estimate the annual seismic moment release due to impacts in teleseismic distances to be in the range of $10^{13}-10^{14} \mathrm{Nm}$. The ranges obtained for faulting induced seismicity are higher than this, but they are global estimates, whereas this estimate only considers impacts in teleseismic distances. Nevertheless, quakes are more likely to occur, but impacts offer the advantage of a known source location.

Smaller impacts are much more likely to occur than large impacts (Hartmann 2005). Teanby (2015) studied the detectability of these smaller impacts. Because the impacts are smaller, they are a weaker seismic source and detection will only be possible in regional distances. Teanby (2015) predicts that the SP will be able to detect 1-3 regional impacts per year.

Besides the seismic signal resulting from quakes, impacts and possibly volcanic activity (Neukum et al. 2004), seismic noise is another source of information that can be included in the investigations. It is especially important to incorporate the seismic noise since the Martian seismic activity is estimated to be lower than on Earth, as seen in Fig. 1.6. As Mars does not posses an ocean, which is one of the major noise sources on Earth (Bonnefoy-Claudet et al. 2006), and in addition no cultural noise produced by human activity will be existing, the overall seismic noise level is assumed to be low, but present. Wind noise was the main signal recorded by the the Viking 2 seismometer (Anderson et al. 1977, Nakamura \& Anderson 1979) and Lognonné \& Mosser (1993) predict that the main noise source will be ground deformations due to wind induced pressure fluctuations. The wind activity observed by the Viking 2 seismometer exhibits regular variations (Nakamura \& Anderson 1979). During the summer, winds are light and show a strong diurnal periodicity. During autumn and spring, winds are stronger and more variable. In the winter the average wind speed is the highest. For all seasons, winds are lower during nighttime, compared to daytime. This diurnal variation was also observed by a mechanical anemometer on the Phoenix Lander (Holstein-Rathlou et al. 2010). During nighttime, wind speeds were lower and less variable in speed and direction compared to daytime. Mimoun et al. (2017) created a noise model for the InSight VBB seismometer, including instrument self-noise, noise in the instrument and ground acceleration generated by environmental effects. Atmospheric pressure fluctuations leading to temperature variations and ground tilt are found to make up the majority of seismic noise recorded by the seismometers in the frequency band of the VBB (0.01-1 Hz). Murdoch et al. (2017b) analyse wind measurements from previous Mars missions and evaluate noise contributions through interactions of the InSight lander with the wind in the same frequency band. They determine that the lander mechanical noise may be detectable on the InSight seismometers, but will be low and not interfere with detections relevant for the mission goals. Resonances of the lander solar panels are estimated at higher frequencies than the bandwidth of the VBB, but might influence the SP. Ground tilt caused by atmospheric pressure fluctuations will also be detectable (Murdoch et al. 2017a). Kenda et al. (2017) examined dust devils on Mars with respect to their seismic signatures. Through modelling, it was concluded that the passage of dust devils and atmospheric vortices creates ground tilt, which could be detectable by the SEIS instrument. Besides wind noise, dust devils are also identified as a source of short-period Rayleigh waves and can be used for probing the shallow subsurface. In general, seismic noise on Mars related to wind will provide additional seismic data to study the planet. 


\subsection{Method}

\subsubsection{Basic concepts}

This section will give a brief overview of the Earth and general seismological concepts and principles. The second part of this section will focus on the method used throughout this work 'Passive Seismic Interferometry.' The information presented in this part is compiled from Fowler (2005) and Shearer (2009) unless otherwise indicated.

Seismology is the study of earthquakes and the journey of elastic waves through the Earth. Here, the focus will be on Earth, but the concepts can be transferred to Mars or any other planetary body. Two different types of seismic waves exist: body waves and surface waves. Body waves travel through the body of the Earth, whereas surface waves travel along its surface, as the names already suggest.

Body waves are separated into two types.

$\boldsymbol{P}$-waves (primary or pressure waves) are compression waves with particle motion in the direction of the wave propagation.

$\boldsymbol{S}$-waves (secondary or shear waves) are transverse waves with particle motion perpendicular to the direction of wave propagation.

The speed of the waves depends on the properties of the material which they travel through. $P$-waves obtain their name from the fact that the $P$-waves travel faster than $S$ waves, therefore arriving first at, for example, a seismometer. $S$-waves cannot propagate through liquids such as the outer core, because liquids cannot sustain a shear.

Surface waves do not penetrate as deep into the interior of the Earth as body waves, because they are constrained to the surface. Their penetration depths depend on their periods. Waves with longer periods penetrate deeper into the Earth than those with shorter periods (Wiggins 1972). Vertical velocity gradients in the Earth's crust and upper mantle, therefore lead to dispersion of the surface waves. Two types of surface waves are distinguished.

Rayleigh waves are characterized by elliptical particle motion with respect to the direction of wave propagation

Love waves are characterized by a transverse and horizontal particle motion.

Surface wave velocities are lower than the body wave velocities, which is why they arrive at seismometers after the $P$ - and $S$-waves. Shallow earthquakes are the best generators of surface waves and their resulting amplitudes are larger compared to the body wave amplitudes. An overview of the different waves can been found in Fig. 1.7

The propagation of these waves depends on the internal structure of the Earth. Like Mars, Earth is composed of the main layers, crust, mantle and core (see Fig. 1.2). The crust is the layer of interest for this work. The crust is the outermost shell of the Earth and is divided into continental and oceanic crust. The average thickness of the continental crust is $38 \mathrm{~km}$, but varies globally. The average $P$-wave velocity of the continental crust is $\sim 6.5 \mathrm{~km} \mathrm{~s}^{-1}$. Its structure and composition are heterogeneous. The oceanic crust is thinner than the continental crust, but has a higher density. Its thickness varies between 


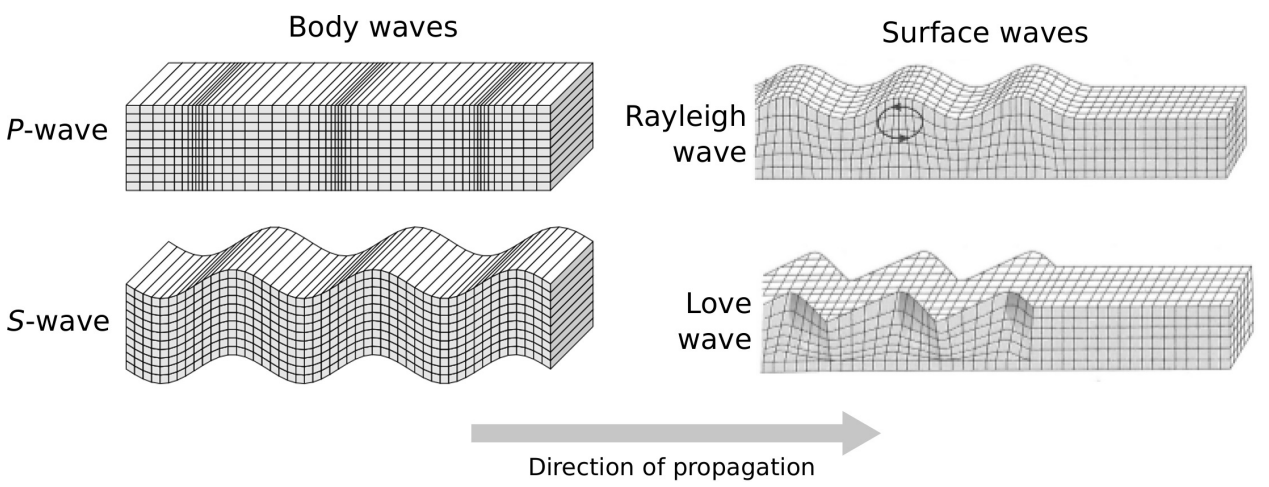

Figure 1.7: Overview of the particle motion for the different seismic wave types. Body waves after Shearer (2009) and surface waves after Fowler (2005).

$5-10 \mathrm{~km}$, with an average P-wave velocity of $\sim 7 \mathrm{~km} \mathrm{~s}^{-1}$. The general composition of continental crust is silica-rich compared to the oceanic crust, which is richer in mafic materials (Mg, Fe-rich). The core forms the innermost part of the Earth and is separated into two regimes, the liquid outer core ( $\sim 2889 \mathrm{~km}$ depth down to $\sim 5150 \mathrm{~km}$ depth) and the solid inner core as the centre. The core is very iron-rich, with the outer core likely being composed of and iron-ally and the inner core being made up of pure iron. The mantle is found between the crust and the core. Its made up of mostly magnesian silicate in the form of olivine. The Mantle and crust are separated by a compositional discontinuity called the Mohorovičić-discontinuity, short 'Moho', named after Andrija Mohorovičić, the first to detect the separation between the crust and the mantle in 1909. Its depth is investigated throughout this work.

In addition to the division into the layers explained above, an additional classification is being used. The lithosphere is considered as the outermost shell of the Earth, which is cool and rigid. It comprises the crust and uppermost mantle and is divided into tectonic plates. The thickness of the lithosphere is very variable and is estimated to vary between $\sim 40-280 \mathrm{~km}$ (Pasyanos 2010, Pasyanos et al. 2014). The mechanically strong lithosphere floats on top of the mechanically weak asthenosphere, which comprises deeper parts of the mantle beneath the lithosphere. Because of high temperatures at asthenospheric depths, the asthenosphere is ductile and viscous flows exist. The lithosphere-asthenosphere boundary (LAB) is a seismic discontinuity characterized by a drop in velocity.

In order to gather information about the different layers, in the case of this work the crust, seismic signals in the form of seismic waves are needed. The main source of seismic waves are earthquakes. An earthquake releases energy, which partially travels in the form of seismic waves through the Earth. Earthquakes occur, with a few exceptions, along plate boundaries with varying temporal intervals, but can in some cases be observed across the Earth. Another source of seismic waves is seismic noise. This source is considered throughout this work. The term noise is used to describe vibrations of the ground not related to earthquakes. In general, seismic noise consists mainly of surface waves, but also body waves have been observed (e.g. Iyer \& Healy 1972, Vinnik 1973). The origin of seismic noise can be natural or cultural, with varying frequency content. At low frequencies, below $1 \mathrm{~Hz}$, the noise is of natural origin, at intermediate frequencies, $1-5 \mathrm{~Hz}$, the sources are of both natural and cultural origin, and at higher frequencies, the sources are cultural. 
Cultural noise exhibits amplitude variations linked to daily and weekly human activity cycles, whereas natural noise shows amplitude variations depending on natural phenomena (Bonnefoy-Claudet et al. 2006). Natural phenomena contributing to the seismic noise are, for example, air pressure variations, wind and flowing water (Iyer \& Healy 1972), whereas cultural noise originates, for example, from traffic and machinery (Campillo \& Roux 2015).

The term 'microseisms' is used to refer to seismic noise of natural origin in general, for example Bonnefoy-Claudet et al. (2006), or to seismic noise in the frequency range of 0.05-0.3 Hz, for example Campillo \& Roux (2015). Microseisms are mainly observed in two frequency bands, the primary microseisms between $0.05-0.08 \mathrm{~Hz}$ and the secondary microseisms between 0.1-0.16 Hz (Schimmel et al. 2011a). The primary microseisms are generated through breaking of ocean gravity waves near the shore and interactions between the waves and the sea floor (Hasselmann 1963). The secondary microseisms are caused by non-linear interference of ocean gravity waves with opposite directions and similar frequencies (Longuet-Higgins 1950, Hasselmann 1963). At long periods, i.e. 30-250 s, the noise is referred to as a 'hum'. This hum is several orders of magnitudes weaker than the microseisms (Haned et al. 2016) and its spectral structure resembles the free background oscillations of the Earth (e.g. Suda et al. 1998). These oscillations are thought to arise from interactions of ocean infragravity waves with the solid Earth (Campillo \& Roux 2015).

\subsubsection{Passive seismic interferometry}

How do we extract useful information from seismic noise? For this purpose we apply the method of passive seismic interferometry, where passive seismic measurements are converted into seismic responses (Wapenaar et al. 2010). The first seismic interferometry theory was already published in 1968 by Claerbout. There it was shown that the autocorrelation of a seismogram of a receiver with a source at depth, i.e. the transmission response, is equal to the reflection seismogram of a surface source and the same receiver. The prerequisites for this result were a 1-D medium bounded by the free surface on top, a homogeneous half-space underneath, and a plane-wave travelling vertically from below. If the actual source at depth were a noise source, then the source wavelet of the reflection response would be the autocorrelation of the noise (Curtis et al. 2006). Wapenaar et al. (2010) summarized the basic principles of seismic interferometry and the following explanations will follow closely their descriptions, unless otherwise indicated.

Fig. 1.8, modified after Schuster (2009), illustrates the basic principle of seismic interferometry. In Fig. 1.8(a), a seismic wave travels from a source at depth through an intermediate layer to a seismic station at the surface and is recorded at some time $t_{\mathrm{a}}$. In Fig. 1.8(b), this wave is reflected downward at the surface and reflected upward again at the intermediate layer interface. It is then recorded at the seismic station at a delayed time $t_{\mathrm{b}}$. By autocorrelating the signal recorded at the station at (a) and (b), indicated by the symbol $*$, the first travel path from the source to the receiver is eliminated. This results in the seismic station being collocated with a virtual source and a wave travelling down from the surface and being reflected upward again. This wave thus reaches the surface at a time proportional to the thickness of the intermediate layer and equal to the difference $t_{\mathrm{b}}-t_{\mathrm{a}}$ (Fig. 1.8c). 

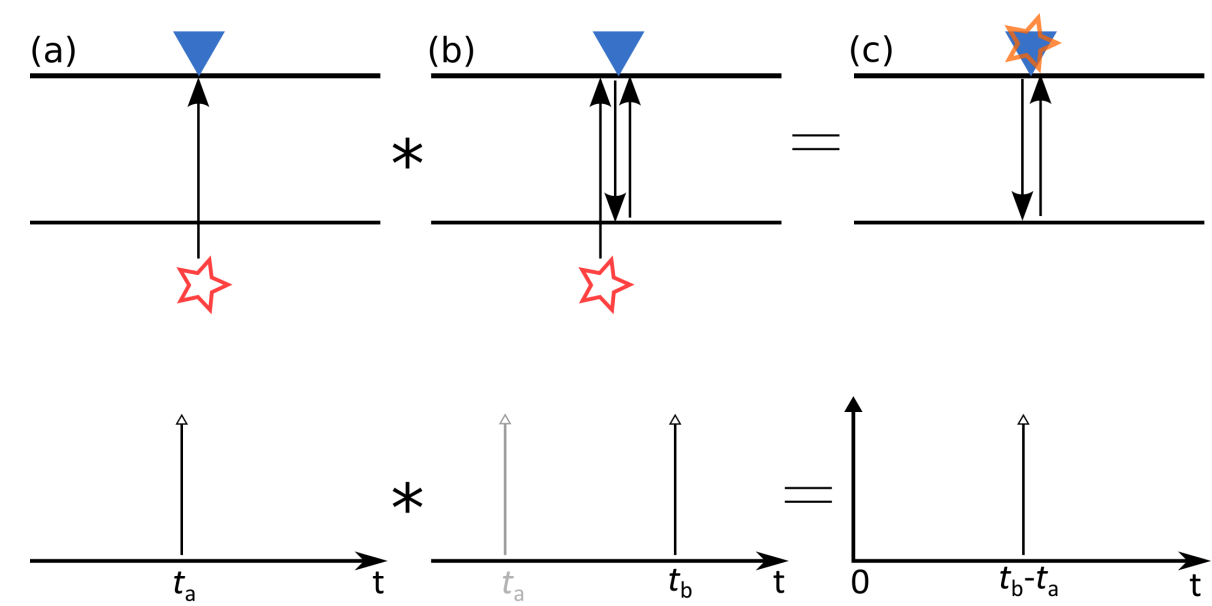

Figure 1.8: Sketch of the theory of seismic interferometry. (a): a seismic wave travels from a source at depth (red) to a seismic station (blue) on the surface and is recorded at time $t_{\mathrm{a}}$. (b): The seismic waves reflected downward at the surface and upward at the interface and recorded at time $t_{\mathrm{b}}$. (c): The autocorrelation of the signal results in the seismic station and a virtual source (orange) being collocated and seismic wave travelling down and being reflected upward again, reaching the station at time $t_{\mathrm{b}}-t_{\mathrm{a}}$. Modified after Schuster (2009).

We assume that the intermediate layer is homogeneous and lossless and it is surrounded by the free surface on top and a homogeneous lossless half-space at the bottom. The intermediate layer would have a transmission coefficient of $\tau$ and a reflection coefficient $r$. The reflection coefficient of the free surface is $r=-1$. A reflection coefficient of -1 means that there is no transmitted pulse, i.e. total internal reflection, so no energy is lost to the free surface. A plane wave propagating vertically upward from a source in the half-space would reach the surface at time $t_{0}=t_{\mathrm{A}}$ (see Fig. 1.8). The first wave arrives at $t=t_{0}$ with amplitude $a_{0}=\tau$. The wave is then reflected from the free surface, and once more at the interface. The reflected wave will reach the seismometer at time $t=t_{0}+\Delta t$ with amplitude $a_{1}=-r \tau$, with $\Delta t=2 \Delta z / v$, and with $\Delta z$ being the thickness of the layer, and $v$ the corresponding wave propagation speed.

The global transmission response $T(t)$ describes the up-going wave-field reaching the surface. It is composed of an infinite time series of impulses, similar to those seen in Fig. 1.8, with regular time intervals $\Delta t$, the first arrival time being $t_{0}$. The amplitudes of these impulses are $a_{\mathrm{i}}=(-r)^{i} \tau$ for $i=0,1,2 \ldots$. For a vertically propagating plane wave, this means that we need to evaluate the autocorrelation of the global transmission response $A=T(t) * T(-t)$. According to Wapenaar et al. (2010), we define the local transmission coefficient for an up-going wave as $\tau=\sqrt{(1-r)(1+r)}=\sqrt{1-r^{2}}$. The autocorrelation at zero lag time is equal to:

$$
\begin{aligned}
A(t) & =\delta(t) \sum_{i=0}^{i=\infty} a_{i}^{2} \\
& =\tau^{2} \delta(t) \sum_{i=0}^{i=\infty} r^{2 i} \\
& =\tau^{2}\left(1-r^{2}\right)^{-1} \delta(t) \\
& =\delta(t) .
\end{aligned}
$$




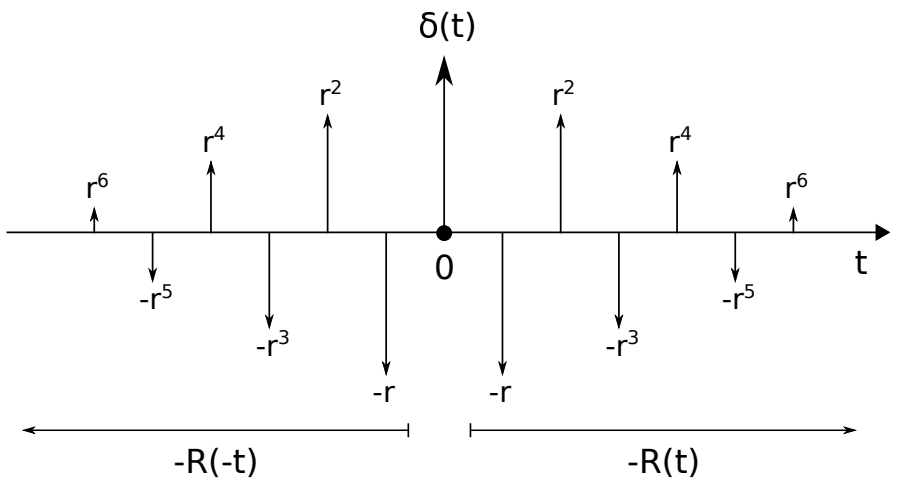

Figure 1.9: Sketch of the autocorrelation $T(t) * T(-t)$ illustrating the associated reflection response $R(t)$. Modified after Wapenaar et al. (2010).

This can be seen as the central impulse at $t=0$ in Fig. 1.9. The autocorrelation for time lag $\Delta t$ is then:

$$
\begin{aligned}
A(t-\Delta t) & =\delta(t-\Delta t) \sum_{i=0}^{i=\infty} a_{i} a_{i+1} \\
& =-r \tau^{2} \delta(t-\Delta t) \sum_{i=0}^{i=\infty} r^{2 i} \\
& =-r \delta(t-\Delta t),
\end{aligned}
$$

which equals the impulse at $\Delta t$ in Fig. 1.9. For integer multiples of $\Delta t$, this results in

$$
A(t-n \Delta t)=(-r)^{n} \delta(t-n \Delta t) \text { for } n=0,1,2 \ldots
$$

These impulses of the causal part of the autocorrelation, without the central impulse at zero time lag, form the negative global reflection response $-R(t)$, of a plane wave travelling down from the free surface. Accordingly, the acausal part of the autocorrelation is then equal to $-R(-t)$ (Fig. 1.9). Summing all this up, we can write the autocorrelation of the global transmission response $T(t) * T(-t)$ as the sum of the central impulse at $t=0$, $\delta(t)$, the causal and acausal global reflection responses, $-R(t),-R(-t)$.

$$
T(t) * T(-t)=\delta(t)-R(t)-R(-t)
$$

which is equal to:

$$
R(t)+R(-t)=\delta(t)-T(t) * T(-t) .
$$

This shows that the global reflection response is attainable from the autocorrelation of the global transmission response.

This approach can then be extended to arbitrary horizontally layered media (Wapenaar 2003). Assuming lossless media, the principle of power conservation holds and can be used to describe the relation of the up and down-going wave-fields. It is easiest to describe the power flux in the frequency domain. The down-going plane wave-field will be represented by $\tilde{D}$ in the frequency domain, with the up-going plane wave-field being $\tilde{U}$. The net down-going power flux will then be $\tilde{D}^{*} \tilde{D}-\tilde{U}^{*} \tilde{U}$, with the superscript asterisk denoting complex conjugation (Wapenaar 2003). An overview of a layered medium with the wave-fields can be found in Fig. 1.10. In the time domain, assuming a plane wave 
Surface

$$
\widetilde{D} \downarrow \uparrow \widetilde{U}
$$

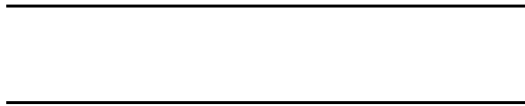

Surface

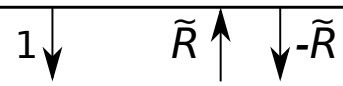

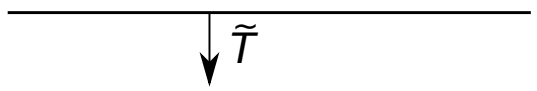

Figure 1.10: Sketch of a layered medium indicating in the frequency domain the down-going plane wave-field $\tilde{D}$, the up-going plane wave-field $\tilde{U}$ on the left and a plane wave travelling down 1 , the up- and down-going reflection responses $\tilde{R}$ and $-\tilde{R}$ and the total down-going transmitted wave-field $\tilde{T}$ on the right.

going down from the free surface, described by $\delta(t)$, the up-going wave-field reaching the surface will be the global reflection response $R(t)$, which will then be reflected downward with a reflection coefficient of -1 . Just below the surface the total down-going wave-field will therefore be $D(t)=\delta(t)-R(t)$ and the up-going wave-field $U(t)=R(t)$, in the frequency domain $\tilde{D}=1-\tilde{R}$ and $\tilde{U}=\tilde{R}$. Using these wave-fields the total down-going power flux becomes:

$$
\begin{aligned}
\tilde{D}^{*} \tilde{D}-\tilde{U}^{*} \tilde{U} & =\left(1-\tilde{R}^{*}\right)(1-\tilde{R})-\tilde{R}^{*} \tilde{R} \\
& =1-\tilde{R}-\tilde{R}^{*} .
\end{aligned}
$$

The total down-going transmitted wave-field (denoted by $\tilde{T}$ ) at the lowest interface includes all surface and internal multiples. There is no up-going wave-field in the lower half-space below the lowest interface, because it is assumed to be homogeneous, therefore the net down-going power flux in the lowest half-space is:

$$
\tilde{D}^{*} \tilde{D}-\tilde{U}^{*} \tilde{U}=\tilde{D}^{*} \tilde{D}=\tilde{T}^{*} \tilde{T} .
$$

Considering the principle of power conservation, eqs 1.6 and 1.7 can be combined to:

$$
1-\tilde{R}-\tilde{R}^{*}=\tilde{T}^{*} \tilde{T}
$$

In the time domain and after rearranging, equation 1.8 becomes:

$$
R(t)+R(-t)=\delta(t)-T(-t) * T(t) .
$$

This is equivalent to equation 1.5 , so the theory holds for any arbitrary horizontally layered media and states that the reflection response can be obtained from the autocorrelation of the transmission response of an impulsive source in the subsurface, independent of its depth.

This result can be altered to include transient or noise signals. If we consider the up-going wave-field at the surface to be $u(t)=T(t) * N(t)$, where $N(t)$ is a noise signal emitted in the lower half-space, then equation 1.5 changes to:

$$
\{R(t)+R(-t)\} * S_{N}(t)=S_{N}(t)-\langle u(t) * u(-t)\rangle .
$$


Here $S_{N}(t)$ is the autocorrelation of the noise and \langle\rangle is the inner product of the two functions. This equation shows that the reflection response of a transient source at the surface can be obtained from the autocorrelation of passive seismic noise. Using seismic interferometry, virtual seismic sources are created in locations with only receivers present, provided noise is present. This result will be used in the following chapters. This 1-D approach can be extended to 2-D and 3-D and it can be shown that the cross-correlation of passive seismic noise equals the reflection response of a transient source at the surface (Wapenaar 2003).

Another approach to the method is by considering seismic interferometry as Green's function retrieval. When referring to Green's function retrieval, here the Green's function is understood as the response of the actual medium to an impulsive point source (Wapenaar \& Fokkema 2006). Wapenaar et al. (2004) derived an exact representation of the Green's function for any inhomogeneous, anisotropic, lossless solid medium. It is shown that the Green's function for such a medium can be obtained from the cross-correlation of two recordings of the wave-field at different receiver positions. By assuming uncorrelated noise sources, this exact approximation can be simplified so that the Green's function can be derived from a direct cross-correlation of the wave-field recorded at two different observation points (Wapenaar \& Fokkema 2006). By decreasing the distance between the two observation points, the limit of the cross-correlation can be obtained resulting in a Green's function version with zero-offset. Kennett (2015) concluded that these two different concepts reinforce each other and give the possibility of making the most of the entire seismic record.

In the following, the approach of obtaining a reflection response from the autocorrelation of a transmission response will be used. Changes in reflectivity can be observed in these reflection responses, coinciding with changes in properties of the subsurface. These reflectivity changes can be identified by, for example, a change in frequency or amplitude. Different layers in the subsurface exhibit different reflective properties, and by identifying the lag times of these reflectivity changes, it is possible to draw conclusions about the structure beneath the seismic station.

Part of the goal of this work is to determine the depth of the transition from crust to mantle, also known as the Moho depth. This transition is marked by an increase in velocity and a change in composition. In the reflection response obtained from the autocorrelation, this is visible as a change in reflectivity. The lag times of this reflectivity change can easily be converted to depth by the simple linear relationship

$$
\Delta z=\frac{v \Delta t}{2},
$$

introduced above. Taking Fig. 1.8 as an example, $\Delta t$ would correspond to $\Delta t=t_{\mathrm{B}}-t_{\mathrm{A}}$ and $\Delta z$ would be the depth of the layer shown. The details on how the autocorrelations are calculated and the reflectivity changes are identified can be found in section 2.3.2.

\subsection{Applications of passive seismic interferometry}

Passive seismic interferometry has been successfully used in a variety of applications. It was first described in 1968 by Claerbout. Later, it became an established method to study 
the interior of the Sun, known as time-distance helioseismology (Duvall Jr. et al. 1993). Weaver \& Lobkis (2001) applied the principle of noise autocorrelations to the ultrasonic domain. Later Campillo \& Paul (2003) were among the first to apply passive seismic interferometry to Earth based seismology. Since then, it has found its way into many different seismological applications and has become an alternative method to approach and evaluate data.

Many studies have employed the method for ambient seismic noise tomography, creating artificial sources in regions with low source coverage with the help of cross-correlations. A few selected studies will be briefly introduced in the following. Shapiro et al. (2005) calculated cross-correlations of ambient seismic noise for stations of the USarray in California and constructed tomographic images of Rayleigh wave group velocities. This research was extended by Lin et al. (2008), who created phase velocity maps for Love and Rayleigh waves for the western US. Stehly et al. (2009) used a similar approach to obtain Rayleigh wave group velocity maps for the western European Alpine region, which were then inverted to obtain a crustal thickness map. A different approach was chosen by Haned et al. (2016), who created a global upper-mantle tomographic model using seismic hum data.

In addition to seismic tomography, the method has been extended to extract seismic body waves. Lin et al. (2013) extracted seismic core phases from cross-correlations of antipodal stations and Xia et al. (2016) observed triplicated $P K P$-phases, where $P K P$ phases are any $P$-phases bottoming in the outer core, using a similar approach. Global body wave propagation was extracted by Nishida (2013) using the seismic hum, whereas Roux et al. (2005) observed $P$-waves on a regional scale.

Several studies have extracted body waves reflected at varying known interfaces from cross-correlations. Draganov et al. (2007) retrieved the reflection response for near surface reflectors from seismic noise. Zhan et al. (2010) obtained Moho reflected shear waves from seismic noise for an array in South Africa, whereas Ruigrok et al. (2011) extracted $P$-wave reflections from reflectors in the crust, the Moho, and possibly upper mantle discontinuities from microseisms. Poli et al. (2012b) observed Moho-reflected $P$ and $S$-waves and Poli et al. (2012a) detected $P$-waves reflected at the $410 \mathrm{~km}$ and $660 \mathrm{~km}$ discontinuities. All these studies benefit from having multiple stations available. In areas where station coverage is low, as will be the case for the InSight mission, it is important to employ methods suitable for one station, for example, in the form of autocorrelations and single-station cross-correlations.

A limited number of studies have considered autocorrelations to extract reflected body waves. Ito et al. (2012) and Ito \& Shiomi (2012) detected Moho reflected body waves and seismic scatterers in the subduction zone north-east of Japan in autocorrelations calculated from vertical component data. Tibuleac \& von Seggern (2012) observed $P$ - and $S$-waves reflected at the crust-mantle boundary in the western US. These reflections were observed on autocorrelations of vertical and horizontal component data. Gorbatov et al. (2013), Kennett et al. (2015) and Sun \& Kennett (2016) mapped the Australian Moho with the help of autocorrelations. Taylor et al. (2016) identified the Moho and other structures in the upper mantle across the North Anatolian Fault zone. Oren \& Nowack (2017) obtained Moho reflected $S$ - and $P$-waves for the central US. Phạm \& Tkalčić (2017) extracted reflected $P$-waves to locate the Moho and an ice-rock interface. Kennett (2015) extended the method to also map the lithosphere-asthenosphere boundary for Australia and Sun 
\& Kennett (2017) traced a mid-lithosphere discontinuity under the North China Craton. Saygin et al. (2017) considered shallower depth and extracted reflected $P$-waves from the bottom of the Jakarta basin. Heath et al. (2018) used autocorrelations to map the upper crustal magmatic structure of a volcano. Most of these studies used seismic noise for the extraction of body waves, only Sun \& Kennett (2016), Sun \& Kennett (2017) and Pham \& Tkalčić (2017) utilized the coda of teleseismic events and Heath et al. (2018) used a combination of both.

Aside from extracting reflected body waves, autocorrelations have been used for other purposes. Wang et al. (2015) calculated autocorrelations from earthquake coda, but to infer information about anisotropy in the inner part of the Earth's inner core. Deen et al. (2017) succeeded in observing the Earth's hum in autocorrelations of ocean bottom seismometer data. Sens-Schönfelder \& Wegler (2006) used auto- and cross-correlations to study seasonal variations of seismic velocities. Wegler \& Sens-Schönfelder (2007) and Ohmi et al. (2008) calculated seismic noise autocorrelations to monitor seismic velocity changes associated with earthquakes. Maeda et al. (2010) monitored changes in autocorrelations and associated them with velocity changes originating from geothermal earthquake swarms. Hobiger et al. (2014) extended the method of autocorrelations to single-station cross-correlations to observe co-seismic velocity changes.

Another planetary body for which seismic data is available is the Earth's moon. During the Apollo missions a seismic stations were set up on the Moon's surface. Passive seismic interferometry has been applied to data from lunar stations. Larose et al. (2005) used lunar seismic noise to extract Rayleigh wave dispersion curves from cross-correlations. Tanimoto et al. (2008) observed diurnal changes in Rayleigh wave group velocity for the same data set. Sens-Schönfelder \& Larose (2010) also identified Rayleigh wave group velocity changes and inferred the shear wave structure in the shallow crust from Rayleigh wave dispersion curves obtained from auto- and cross-correlations. All of these studies consider data from the Apollo 17 network. Nishitsuji et al. (2016) used body-wave seismic interferometry to detect the lunar Moho from the $P$-wave coda of deep moonquakes, considering data from long-period stations set up during Apollo 12, 14, 15 and 16. These studies give hope that passive seismic interferometry can be applied to other planetary bodies, such as Mars.

As can be seen, the method of passive seismic interferometry offers a wide variety of applications. The following chapters will focus on the extraction of the crustal thickness from seismic noise auto-correlations and single-station cross-correlations. 



\section{Crustal thickness from vertical component ambient seismic noise data}

In the middle of difficulty lies opportunity.

Albert Einstein

The contents of this chapter have been published as "Crustal thickness across the Trans-European Suture Zone from ambient noise autocorrelations" in 2018 in Geophysical Journal International vol. 212, pages 1237-1254. The work, writing and creation of figures were carried out by myself, under the supervision of B. Knapmeyer-Endrun, from the Max Planck Institute for Solar System Research.

\subsection{Summary}

We derive autocorrelations from ambient seismic noise to image the reflectivity of the subsurface and to extract the Moho depth beneath the stations for two different data sets in Central Europe. The autocorrelations are calculated by smoothing the spectrum of the data in order to suppress high amplitude, narrow-band signals of industrial origin, applying a phase autocorrelation algorithm and time-frequency domain phase-weighted stacking. The stacked autocorrelation results are filtered and analysed predominantly in the frequency range of 1-2 Hz. Moho depth is automatically picked inside uncertainty windows obtained from prior information. The processing scheme we developed is applied to data from permanent seismic stations located in different geological provinces across Europe, with varying Moho depths between 25 and $50 \mathrm{~km}$, and to the mainly short-period temporary PASSEQ stations along seismic profile POLONAISE P4.

The autocorrelation results are spatially and temporarily stable, but show a clear correlation with the existence of cultural noise. On average, a minimum of six months of data is needed to obtain stable results. The obtained Moho depth results are in good agreement with the subsurface model provided by seismic profiling, receiver function estimates, and the European Moho depth map. In addition to extracting the Moho depth, it is possible to identify an intracrustal layer along the profile, again closely matching the seismic model. 
For more than half of the broad-band stations, another change in reflectivity within the mantle is observed and can be correlated with the lithosphere-asthenosphere boundary to the west and a mid-lithospheric discontinuity beneath the East European Craton.

With the application of the developed autocorrelation processing scheme to different stations with varying crustal thicknesses, it is shown that Moho depth can be extracted independent of subsurface structure, when station coverage is low, when no strong seismic sources are present, and when only limited amounts of data are available.

\subsection{Introduction}

Passive seismic interferometry is a powerful tool to learn more about the subsurface from the seismic background wavefield. Already in 1968, Claerbout showed that the autocorrelation of a vertically incident seismic wave transmitted through a layered medium equals the reflection response of that medium. Wapenaar et al. (2004) extended the approach to 3-D inhomogeneous media. Shapiro \& Campillo (2004) and Shapiro et al. (2005) showed that the cross-correlation of seismograms at two receivers equals the Green's function of the Earth's structure between them. Over the last years, cross-correlating ambient seismic noise data to obtain surface wave dispersion has become a well-established method, for example in ambient seismic noise tomography (e.g. Shapiro et al. 2005, Lin et al. 2008, Stehly et al. 2009, Haned et al. 2016). This method was also extended to seismic body waves on a global (e.g. Lin et al. 2013, Nishida 2013, Xia et al. 2016) as well as on a local crustal scale (Roux et al. 2005). Moho-reflected $P$ - and $S$-waves have successfully been extracted from cross-correlations in several studies (Zhan et al. 2010, Ruigrok et al. 2011, Poli et al. 2012b).

Recently not only cross-correlations, but also autocorrelations have been used to retrieve body wave reflections from seismic noise. Ito \& Shiomi (2012) calculated autocorrelations to identify seismic scatterers within the subducting slab close to Japan. Tibuleac $\&$ von Seggern (2012) detected body wave reflections from the crust-mantle boundary using continuous waveforms from USArray stations in Nevada. Similar methods to derive crustal thickness from autocorrelations have been applied to data from Australia (Gorbatov et al. 2013, Kennett et al. 2015), the area of the North Anatolian Fault in Turkey (Taylor et al. 2016), and the central US (Oren \& Nowack 2017). Kennett (2015) extended the method to larger depths to also trace the lithosphere-asthenosphere boundary across Australia, whereas Saygin et al. (2017) applied it to shallower structure to map the $P$-wave reflectivity of the Jakarta basin. Nishitsuji et al. (2016) applied seismic interferometry to image the lunar Moho with the help of autocorrelations. Instead of ambient noise, they used the $P$-wave coda of deep moonquakes. A similar approach using $P$ - and $S$-wave coda from teleseismic earthquakes was employed by Sun \& Kennett (2016) and Sun \& Kennett (2017).

The methods applied to calculate autocorrelations often vary, as processing influences the results and depends on the data set used (Fichtner et al. 2017). One of the main differences in previous studies is the frequency band considered. The filters applied vary from 0.2-0.4 Hz (Taylor et al. 2016) to 2-4 Hz (Gorbatov et al. 2013, Kennett et al. 2015, Saygin et al. 2017) and to a variety of ranges in between. The actual autocorrelations are calculated using different methods, for example by using a regularized transfer function 
approach (Gorbatov et al. 2013, Kennett et al. 2015, Kennett 2015, Saygin et al. 2017), or applying a phase autocorrelation algorithm (Taylor et al. 2016). Autocorrelations are calculated for various window lengths, from $1 \mathrm{hr}$ (Ito \& Shiomi 2012, Tibuleac \& von Seggern 2012, Taylor et al. 2016, Oren \& Nowack 2017) to the whole duration of the recording (Saygin et al. 2017). Different pre- and post-processing steps such as 1-bit normalization (Ito \& Shiomi 2012, Tibuleac \& von Seggern 2012, Oren \& Nowack 2017), spectral whitening (Oren \& Nowack 2017), or automatic gain control (Tibuleac \& von Seggern 2012, Oren \& Nowack 2017, Saygin et al. 2017) are also used. Saygin et al. (2017) applied phase-weighted stacking, whereas in all other studies, linear stacking was used. The identification of subsurface structures was based on identifying coherent signals across stations and amplitude maxima in the autocorrelation (Ito \& Shiomi 2012, Tibuleac \& von Seggern 2012, Gorbatov et al. 2013, Taylor et al. 2016, Oren \& Nowack 2017), or identifying reflectivity changes (Kennett et al. 2015, Kennett 2015).

Here we use seismic noise data to detect reflectivity changes associated with the Moho using autocorrelations. This method is tested on data from stations across central and eastern Europe with well-known but variable crustal thickness. The usage of autocorrelations is of special interest when only a single station is available, for example in planetary missions like the InSight (Interior Exploration using Seismic Investigations, Geodesy and Heat Transport) mission to Mars (Banerdt et al. 2013) and a proposed lander mission to Europa (Panning et al. 2017b).

\subsection{Data and method}

\subsubsection{Data}

In development of the method, vertical component data of 25 broad-band stations across central and eastern Europe, along with stations of the German Experimental Seismic System (GERESS) array are processed (Table 2.1). In general, $3 \mathrm{yr}$ of continuous data are used. The stations are located in different geologic settings with varying crustal thickness and subsurface structure. The geologic regions cover the Rhine Graben, the North German and Polish Basins, the Central European Platform, the Bohemian Massif and the East European Craton. The Rhine Graben is part of the European Cenozoic Rift system (Ziegler 1992) with a shallow Moho (Brun et al. 1992) and asthenosphere (Glahn \& Granet 1992). The basins are part of the Central European Basin System and contain low velocity sediments $\left(v_{\mathrm{p}}<5 \mathrm{~km} \mathrm{~s}^{-1}\right)$ to depths of up to $10 \mathrm{~km}(\mathrm{Grad}$ et al. 2003, Lamarche \& Scheck-Wenderoth 2005, Scheck-Wenderoth \& Lamarche 2005). The Bohemian Massif is an outcrop of the crystalline basement of the Variscan orogeny, which can be subdivided into different terranes of different tectonic origins (Matte et al. 1990, Schulmann et al. 2009). Each unit is characterized by different fabric in the mantle lithosphere (Babuška \& Plomerová 2013). The Eger Rift, located in the Saxothuringian terrane of the Bohemian Massif, is also part of the European Cenozoic Rift System. The Central European Platform consists of remains of the Variscan orogeny with a homogeneous crustal thickness (Artemieva \& Meissner 2012, Meier et al. 2016), whereas the East European Craton is of Precambrian origin, stable since at least $1.45 \mathrm{Ga}$, and overlain by a younger thin sedimentary cover (Bogdanova et al. 2006). An overview of the geologic provinces of Central 
Europe along with the broad-band stations used and the Moho depth map according to Grad et al. (2009) can be seen in Fig. 2.1. Moho depth below the stations varies between 25 and $50 \mathrm{~km}$.

The GERESS array is a small array composed of four concentric rings, the biggest one with a diameter of $4 \mathrm{~km}$, located on the Moldanubian of the Bohemian Massif at the boundary between Germany and the Czech Republic (Harjes 1990). It consists of 25 short-period stations (GS-13, 1 s eigenperiod) with mostly vertical-component sensors. In addition to that, at one station (GEC2) a three-component broad-band (STS-2, $120 \mathrm{~s}$ eigenperiod) and a vertical-component short-period seismometer are collocated. The layout of the array can be seen in Appendix A2.15. With the developed method changes in reflectivity corresponding to the crust-mantle transitions are extracted from autocorrelations for the broad-band and the GERESS array stations and compared to estimates obtained from receiver functions (Knapmeyer-Endrun et al. 2014) and combinations of seismic and gravity data (Grad et al. 2009).

The method developed in the context of this work is then applied to data from the temporary, mostly short-period, seismic stations of the PASSEQ network (Grad et al. 2003, Wilde-Piórko et al. 2006, 2008) along the POLONAISE'97 P4 seismic profile across Eastern Germany, Poland and Lithuania (Guterch et al. 1999, Grad et al. 2003). Seismic profiling along this line provides a detailed model of the subsurface structures. Crustal thickness varies between $30-50 \mathrm{~km}$ from west to east with a distinct increase across the Trans-European Suture Zone between Phanerozoic Europe and the East European Craton. Data availability for the PASSEQ stations varies between days and up to $3 \mathrm{yr}$ of continuous data, but only stations with more than 6 months of available data were used.

\subsubsection{Method}

As detailed above, the methods applied to calculate autocorrelations often vary, for example, with respect to frequency band, pre-processing, correlation method and type of stacking. Here we combine different processing steps in the following way. Before starting with the actual processing, the data are downsampled to $10 \mathrm{~Hz}$. The vertical component data are then cut into $3 \mathrm{hr}$ time windows, where the mean and linear trend are removed. In some cases, mostly for short-period stations, only a few $3 \mathrm{hr}$ time windows are available due to data gaps. For those stations, the time window length is reduced to $1 \mathrm{hr}$. The results are very similar, for example, for station GEC2 the correlation coefficient for stacked autocorrelations obtained with 1 and $3 \mathrm{hr}$ time windows with a lag time of 3.5-25 s is 0.965 . This lag time window excludes the central peak and first sidelobes as well as later parts of the autocorrelation without significant signal. In the complete lag time window considered of 0-30 s, the correlation coefficient is even 0.9999 (Fig. A2.17). Lag time is defined as the time delay with respect to the maximum central peak of the autocorrelation. The data are tapered and a fourth-order Butterworth high pass filter with corner frequency of $2 \mathrm{~s}$ is applied. Time windows with data gaps or spikes are eliminated to ensure data quality. Afterwards, the amplitude spectrum for each time window is smoothed to eliminate, possibly dominating, very narrow-band signals with an industrial origin, for example, the machine related peak at $2.083 \mathrm{~Hz}$ observed across most of Germany, with one source located near the Czech border (Bokelmann \& Baisch 1999). About $10 \mathrm{yr}$ after this study, this signal is still clearly visible in the data (Fig. 2.2b) and still most prominent at station 
Table 2.1: List of broad-band stations used, along with time period of data analysed and geologic province. CH - Switzerland Seismological Network; CZ - Czech Regional Seismic Network; EE Estonian Seismic Network; GE - GEOFON; GR - German Regional Seismic Network; PL - Polish Seismological Network; SX - Saxon Seismic Network

\begin{tabular}{lccccl}
\hline $\begin{array}{l}\text { Station } \\
\text { Name }\end{array}$ & $\begin{array}{c}\text { Network } \\
\text { Code }\end{array}$ & $\begin{array}{c}\text { Longitude } \\
{\left[{ }^{\circ}\right]}\end{array}$ & $\begin{array}{c}\text { Latitude } \\
{\left[{ }^{\circ}\right]}\end{array}$ & Data used & $\begin{array}{l}\text { Geologic } \\
\text { Province }\end{array}$ \\
\hline BEL & PL & 20.7920 & 51.8370 & $2009-2011$ & Polish Basin \\
BFO & GR & 8.3296 & 48.3301 & $2006-2008$ & Rhine Graben \\
BRG & GR & 13.9428 & 50.8732 & $2006-2008$ & Bohemian Massif \\
BSEG & GR & 10.3169 & 53.9353 & $2006-2008$ & North-German Basin \\
BUG & GR & 7.2693 & 51.4406 & $2006-2008$ & Central European Platform \\
CLL & GR & 13.0026 & 51.3077 & $2007-2009$ & Bohemian Massif \\
CLZ & GR & 10.3724 & 51.8416 & $2006-2008$ & Central European Platform \\
FBE & SX & 13.3540 & 50.9210 & $2006-2008$ & Bohemian Massif \\
GEC2 & GR & 13.7016 & 48.8451 & $2006-2008$ & Bohemian Massif \\
GKP & PL & 17.2367 & 53.2697 & $2006-2008$ & Polish Basin \\
KHC & CZ & 13.5782 & 49.1309 & $2006-2008$ & Bohemian Massif \\
KRUC & CZ & 16.3952 & 49.0619 & $2006-2008$ & Bohemian Massif \\
MORC & CZ & 17.5428 & 49.7766 & $2006-2008$ & Bohemian Massif \\
MOX & GR & 11.6156 & 50.6447 & $2006-2008$ & Bohemian Massif \\
PABE & GE & 23.9680 & 55.5052 & $2013-2015$ & East European Craton \\
PBUR & GE & 21.9294 & 56.0236 & $2013-2015$ & East European Craton \\
SLIT & GE & 22.2905 & 57.6287 & $2008-2010$ & East European Craton \\
STU & GE & 9.1933 & 48.7708 & $2006-2008$ & Rhine Graben \\
SULZ & CH & 8.1115 & 47.5275 & $2006-2008$ & Rhine Graben \\
SUW & PL & 23.1808 & 54.0125 & $2006-2008$ & East European Craton \\
TNS & GR & 8.4473 & 50.2225 & $2006-2008$ & Rhine Graben \\
UBBA & GR & 10.0010 & 50.8188 & $2006-2008$ & Central European Platform \\
VSU & EE & 26.7347 & 58.4620 & $2006-2008$ & East European Craton \\
WET & GR & 12.8782 & 49.1440 & $2006-2008$ & Bohemian Massif \\
WLF & GE & 6.1526 & 49.6646 & $2006-2008$ & Central European Platform \\
\hline & & & & & \\
\end{tabular}




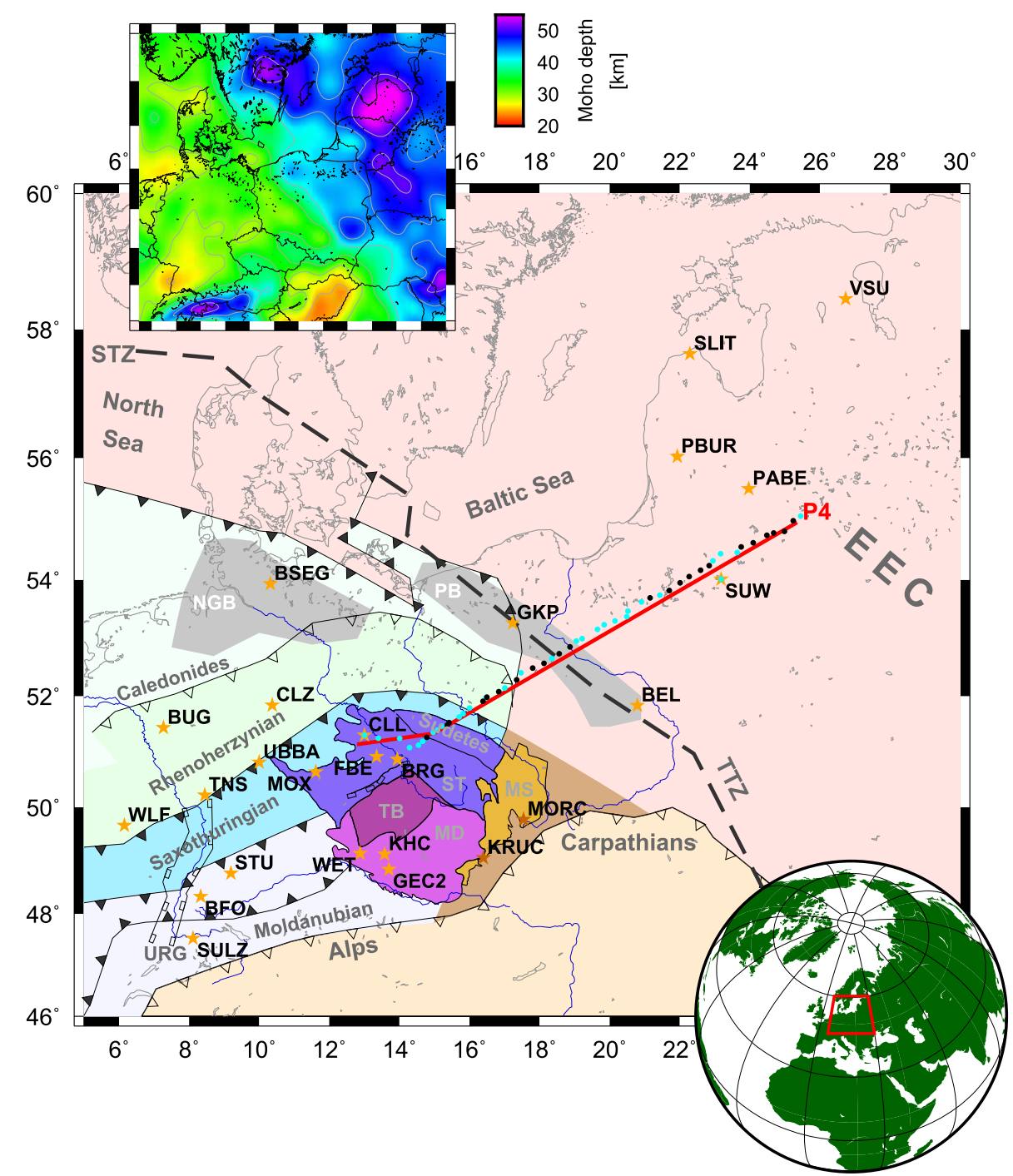

Figure 2.1: Simplified overview of tectonic setting in central Europe (modified after KnapmeyerEndrun et al. (2014)), along with the stations used. Upper inset shows the Moho map created by Grad et al. (2009). Lower inset: Location of study area. Orange stars: broad-band stations used. Cyan dots: PASSEQ stations used. Black dots: PASSEQ stations along profile P4 not included. Labels: EEC, East European Craton; MD, Moldanubian of Bohemian Massif; MS, Moravo-Silesian of Bohemian Massif; NGB, North German Basin; PB, Polish Basin. ST, Saxothuringian of Bohemian Massif; STZ, Sorgenfrei-Tornquist Zone; TB, Tepla-Barrandian of Bohemian Massif; TTZ, Teisseyre-Tornquist-Zone; URG, Upper Rhine Graben 

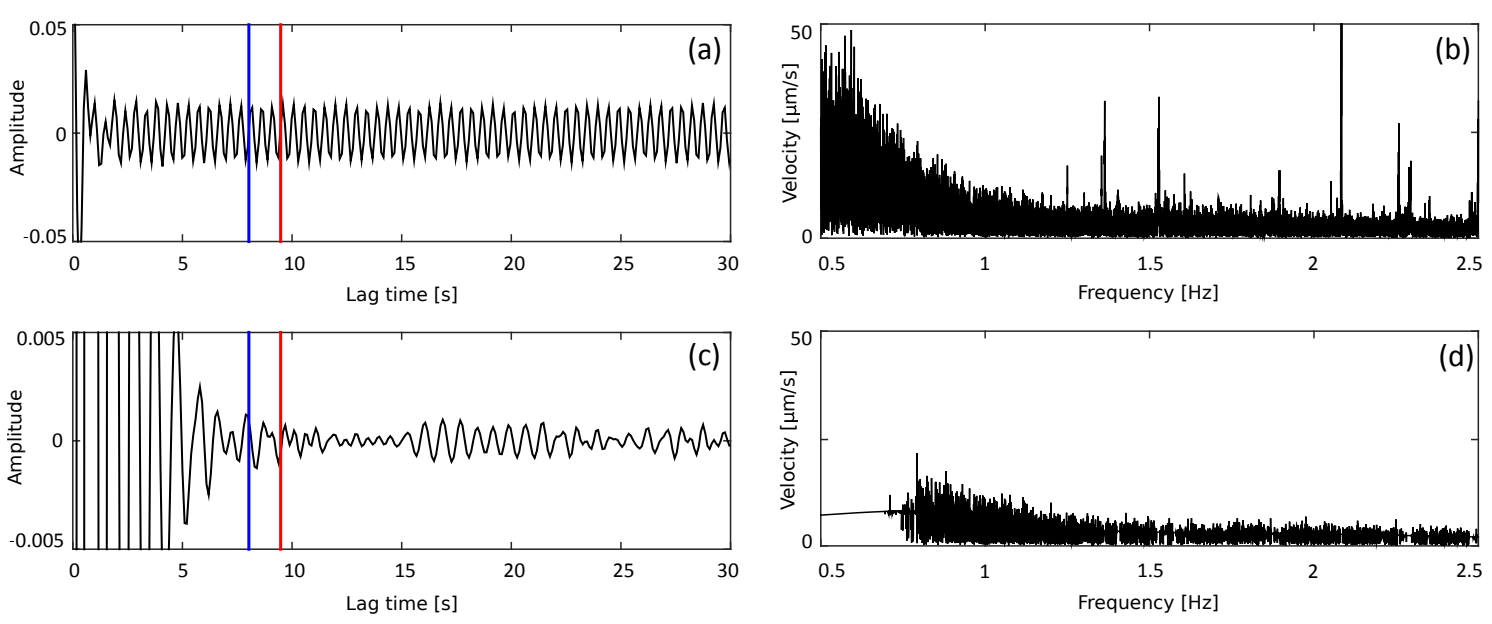

Figure 2.2: Example of spectral smoothing. (a) and (c) show tf-PWS stacked autocorrelation result, filtered between 1 and $2 \mathrm{~Hz}$, for station TNS without and with spectral smoothing. (b) shows a frequency spectrum for an example $3 \mathrm{hr}$ time window of data after high pass filtering and subtraction of the mean and trend. (d) shows the frequency spectrum for the same time window after additional spectral smoothing. Red and blue lines indicate calculated lag time of Moho reflected $P$-waves according to Grad et al. (2009) and Knapmeyer-Endrun et al. (2014), respectively.

TNS. In order to suppress the spikes in the spectrum, we calculate moving averages over a long and a short frequency window. The window lengths have been chosen as 10 samples for the short and 10000 samples for the long window, where each sample is equal to $4.63 \times 10^{-5} \mathrm{~Hz}$. For each data point, the ratio between long and short term window is calculated, and if it is smaller than the mean ratio for the entire spectrum, the spectral amplitude at the data point is replaced by the long term average, thereby smoothing the spectrum (Fig. 2.2).

For each time window, we then calculate the phase autocorrelation (Schimmel 1999). This method has, for example, been applied to seismic noise cross-correlations by Schimmel et al. (2011b). Employing the phase autocorrelation ensures that the results are independent of the amplitude, meaning that high-amplitude transient events such as earthquakes do not influence the result. To compute the phase autocorrelation, the data are Hilbert transformed and the instantaneous phase is extracted. From these phases the autocorrelations are calculated according to Schimmel (1999):

$$
c_{\mathrm{pac}}(t)=\frac{1}{2 T} \sum_{\tau=\tau_{0}}^{\tau_{0}+T}\left\{\left|e^{i \Phi(t+\tau)}+e^{i \Phi(\tau)}\right|^{v}-\left|e^{i \Phi(t+\tau)}-e^{i \Phi(\tau)}\right|^{v}\right\},
$$

where $c_{\text {pac }}$ is the phase autocorrelation, $t$ the lag time and $\Phi(\tau)$ the instantaneous phase. The exponent $v$ can be used to increase the signal-to-noise ratio. Here we set $v=1$. This results in a number of autocorrelation functions, which are then stacked in a timefrequency domain phase weighted stack (tf-PWS; Schimmel \& Gallart 2007). The tf-PWS is an extension of the phase weighted stack (PWS; Schimmel \& Paulssen 1997), where every sample of the linear stack is weighted by the amplitude-unbiased phase stack:

$$
c_{\mathrm{ps}}(t)=\left|\frac{1}{N} \sum_{j=1}^{N} e^{i \Phi_{j}(t)}\right|^{\mu} .
$$



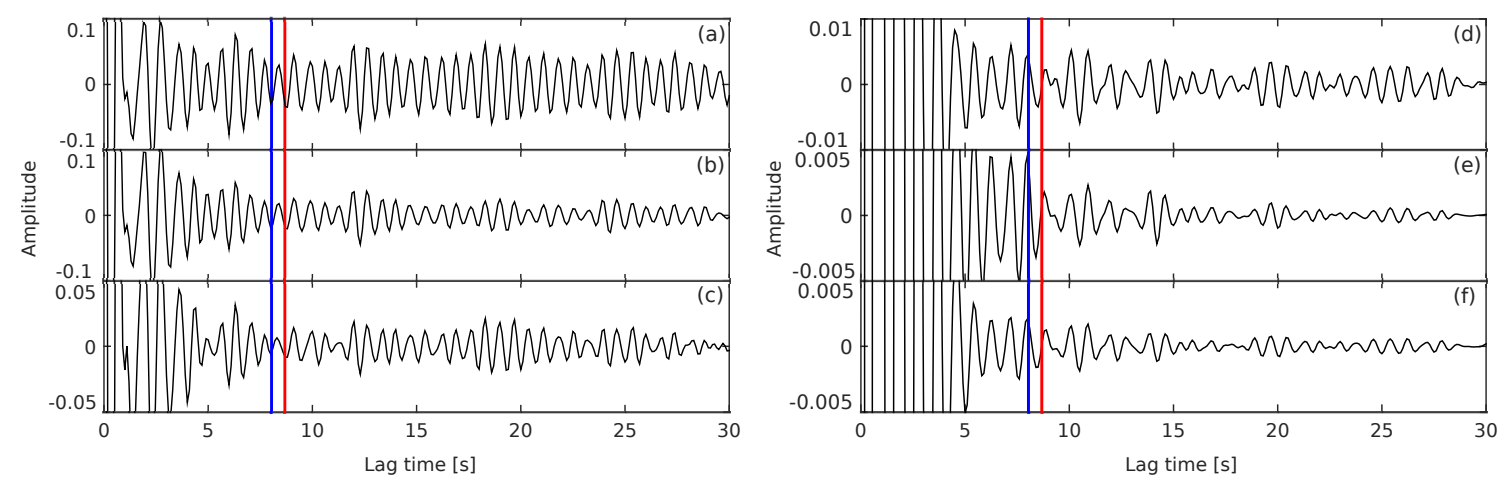

Figure 2.3: Application of different stacking algorithms to autocorrelations for station BFO. All results are filtered between 1-2 Hz. Left: stacking applied to autocorrelations without spectral smoothing; right: autocorrelations calculated with spectral smoothing. (a,d): Linear stack; (b,e): phase-weighted stack according to eq. 2.2 (Schimmel \& Paulssen 1997); (c,f): time-frequency domain phase weighted stack. Red and blue lines indicate the calculated lag times of Moho reflected $P$-waves according to Grad et al. (2009) and Knapmeyer-Endrun et al. (2014).

The tf-PWS makes use of the $S$-transform (Stockwell et al. 1996), which gives the timefrequency decomposition of the computed autocorrelations. The time-frequency phase stack is computed according to Schimmel \& Gallart (2007) as

$$
c_{\mathrm{tf}-\mathrm{ps}}(\tau, f)=\left|\frac{1}{N} \sum_{j=1}^{N} \frac{S_{j}(\tau, f) e^{i 2 \pi f \tau}}{\left|S_{j}(\tau, f)\right|}\right|^{\alpha},
$$

where $c_{\mathrm{tf}-\mathrm{ps}}$ is the time-frequency phase stack and $S_{j}(\tau, f)$ is the $S$-transform of the $j$-th phase autocorrelation. The exponent $\alpha$ is used to sharpen the transition between coherent and incoherent signal summation (Schimmel et al. 2011b). $\alpha=1$ is used throughout this study. Afterwards, the tf-PWS $\left(S_{\text {pws }}(\tau, f)\right)$ is obtained by multiplication of the timefrequency phase stack with the $S$-transform of the linear stack of all phase autocorrelations $\left(S_{\text {ls }}(\tau, f)\right)$. The time-frequency phase stack is hereby used as a measure to down-weigh incoherent parts of the linear stack, which reduces the overall amplitudes because $c_{\mathrm{tf}-\mathrm{ps}}$ varies between 0 and 1 (Fig. 2.3).

$$
S_{\mathrm{pws}}(\tau, f)=c_{\mathrm{tf}-\mathrm{ps}}(\tau, f) S_{\mathrm{ls}}(\tau, f)
$$

As a final processing step, the inverse $S$-transform is applied to obtain the tf-PWS in the time domain.

After obtaining the stacked result, the autocorrelation is filtered between 1 and $2 \mathrm{~Hz}$ with a fourth-order Butterworth filter (Fig. 2.4). This gives the final result, which is then used to determine the Moho depth by picking changes in reflectivity in an automated approach. Especially in areas with a gradational Moho and no distinct reflector it is helpful to look for the Moho as the base of the crustal reflector (Kennett 2015). In a first step the envelope of the autocorrelation stack is calculated. Afterwards the second derivative of the envelope is calculated and weighted with a five second moving average of the envelope. Maxima in the second derivative coincide with the largest changes in slope of the envelope. Weighting the second derivative retains the amplitude information of the autocorrelation, assigning higher relevance to high amplitude parts. Local maxima of 


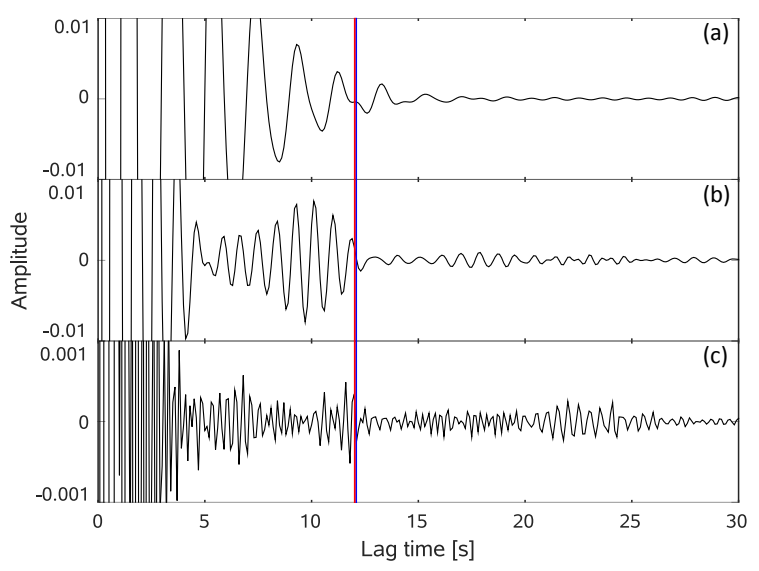

Figure 2.4: Different filtering bands applied to the autocorrelation for station GEC2. Frequency bands used: (a): $0.5-1 \mathrm{~Hz},(\mathrm{~b}): 1-2 \mathrm{~Hz},(\mathrm{c}): 2-4$. Red and blue lines (here overlapping) indicate calculated lag time of Moho reflected $P$-waves according to Grad et al. (2009) and KnapmeyerEndrun et al. (2014).

the weighted second derivative are picked in a time window based on prior information. This time window is calculated as two-way vertical $P$-wave traveltime from the Moho. Moho depth and associated uncertainty at each station location are taken from Grad et al. (2009). Average crustal $P$-wave velocities are taken from Majdański (2012) for Poland, Karousová et al. (2012) for the Czech Republic and Molinari \& Morelli (2011) for the remaining area, and a $5 \%$ velocity uncertainty is assumed. The highest maximum of the weighted second derivative in the time window defined by these values is selected as Moho depth (Fig. 2.5), after visual comparison to the autocorrelation to ensure that a reflectivity change is visible. The lag time chosen for the Moho is converted to depth using the average crustal velocities as described above. The same approach is used when converting the Moho depths provided by Grad et al. (2009) and Knapmeyer-Endrun et al. (2014) to lag time.

For the stations on the profile $\mathrm{P} 4$ an uncertainty window is defined by a $2 \mathrm{~km}$ depth uncertainty, also used by Grad et al. (2009) for seismic data, plus a $3 \%$ uncertainty of the velocity values provided by seismics (Grad et al. 2003). Here again the highest maximum in this uncertainty window is chosen as Moho depth. This pick is compared to the waveform of the autocorrelation and if a prominent reflectivity change is visible is accepted as Moho depth. If no clear reflectivity change is detectable inside the uncertainty window, we additionally consider the frequency band 2-4 Hz following Sun \& Kennett (2017), who compare autocorrelations in various frequency bands.

\subsection{Results}

\subsubsection{Spatial consistency}

The GERESS array provides the possibility to compare results for stations located very closely together, as well as results for different seismometer types. Due to the small size of the array, the same subsurface structures are present for all stations, which provides a check for spatial consistency of the autocorrelations. Because many stations contained 

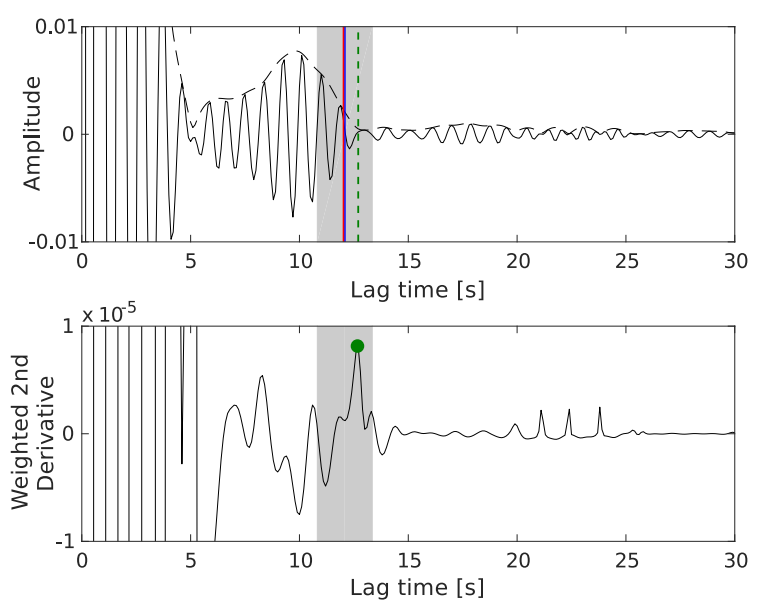

Figure 2.5: Upper image shows stacked and filtered (1-2 Hz) autocorrelation result for station GEC2 with the envelope in dashed black and the highest maximum of the weighted second derivative (bottom panel) in dashed green. The time window, expected to contain the Moho reflection, based on prior information, is marked in grey. Red and blue lines (overlapping) indicate calculated lag time of Moho reflected $P$-waves according to Grad et al. (2009) and Knapmeyer-Endrun et al. (2014).

data gaps, the time window length used for the autocorrelations is decreased to $1 \mathrm{hr}$. In order to compare the short-period results with the broad-band seismometer located at station GEC2, the instrument response is removed as a first processing step. The removal of the instrument response does not change the main features of the autocorrelation such as maxima, minima or interpretation by automatically selecting reflectivity changes, but it causes subtle differences (Fig. A2.16). This step is necessary to allow the direct comparison of the two seismometer types.

Fig. 2.6 shows the autocorrelation results for all stations of the array from the inner ring to the outer rings. The same main features are visible in all autocorrelations. The changes in amplitude at around 5, 12 and $16 \mathrm{~s}$ can clearly be seen throughout the array. Some stations, for example, GED9, vary in the overall shape, but still contain the same features as mentioned above. The average correlation coefficient between all stations for the lag time of 3.5-25 s is 0.987 , indicating high spatial consistency.

At location GEC2 of the GERESS array a short-period and a broad-band seismometer are collocated. Fig. 2.6 shows results for the two sensors in blue and red (red: broad-band, blue: short-period seismometer). The results are very similar and show the same features and a very high correlation coefficient of 0.999 for the lag time of 3.5-25 s.

\subsubsection{Temporal properties and consistency}

When comparing daily stacked autocorrelations, it becomes apparent that the details of the autocorrelations vary with time (Fig. 2.7). The main features of the autocorrelations, for example, at around $8 \mathrm{~s}$ for CLL and $17 \mathrm{~s}$ for PBUR, are consistent across the examined time period (Fig. $2.7 \mathrm{a}$ and b), but additional features appear and disappear. This was also observed by Tibuleac \& von Seggern (2012) for autocorrelations in Nevada. At station CLL, the general amplitude of the signal is higher during the middle of the year (northern summer), compared to the season around the end and beginning of the years 


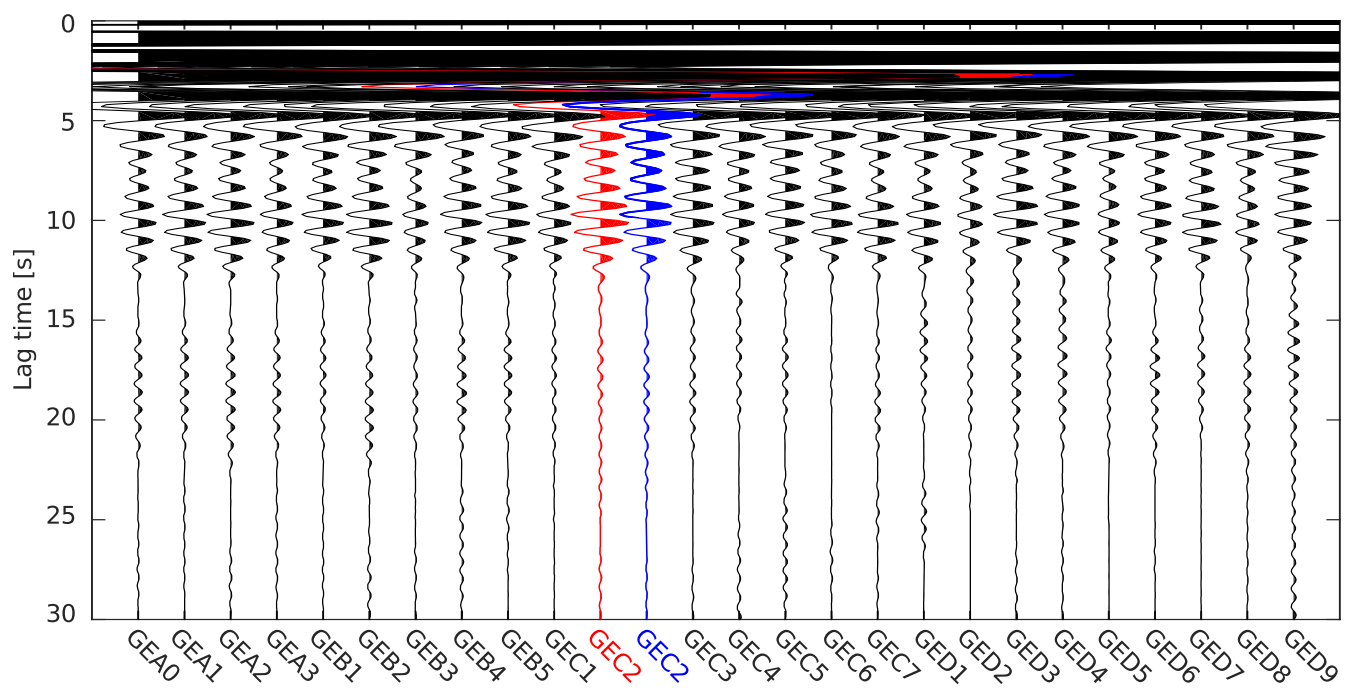

Figure 2.6: Comparison of autocorrelation results for the stations of the GERESS array, filtered between 1 and $2 \mathrm{~Hz}$. Red and blue traces depict the comparison of collocated broad-band and short-period seismometer, respectively.

(northern winter), which is also the case for most of the other stations. At station PBUR the amplitudes toward the end of the years are higher than during the summer season. This can also be observed for four other stations (BSEG, SUW, SLIT, VSU).

Figs 2.7(c) and (d) show the variances between 0-30 s for each autocorrelation against time. For station CLL, an increase in variance during the middle of the years can clearly be observed, whereas station PBUR shows slightly higher variances toward the end of the years, although the variation is not as clear. Several other stations also show higher variances during the winter season. The Lomb-Scargle method (Lomb 1976, Scargle 1982) is applied to estimate power spectra from this time series of variances. It is used to account for data gaps and potentially unequally sampled data. These power spectra (Figs 2.7e and f) show clear peaks at distinct frequencies, which can be associated with yearly, weekly and daily variations. For station CLL the highest peak belongs to the frequency of one year (Fig. 2.7e). This also coincides with the variation in amplitude visible in the Fig. 2.7(a). The peak at around $1.7 \mu \mathrm{Hz}$ is equal to one week, but with a much smaller amplitude and is therefore not visible in the daily stacks. The peak at $11.6 \mu \mathrm{Hz}$ coincides with one day, but cannot be resolved in the daily stacks. The red line indicates the $99 \%$ probability threshold for the peak not to be a random fluctuation and all of these distinct frequency peaks are clearly above this threshold. Not all of these three frequency peaks are observable for all stations. Data from some stations are clearly dominated by only one frequency and harmonics thereof (e.g. station PBUR, Fig. 2.7f). Only the frequency peak for one day is visible in the spectra for all stations with varying amplitude. Some stations show additional frequency peaks, which cannot easily be associated with any obvious time interval.

With respect to limited data availability, for example, from temporal networks, it is important to know how much data is required to obtain a stable result. To learn more about the required amount of data, the root-mean-square (RMS) value of the difference between the result of linear stacking of the single autocorrelations over increasing time 

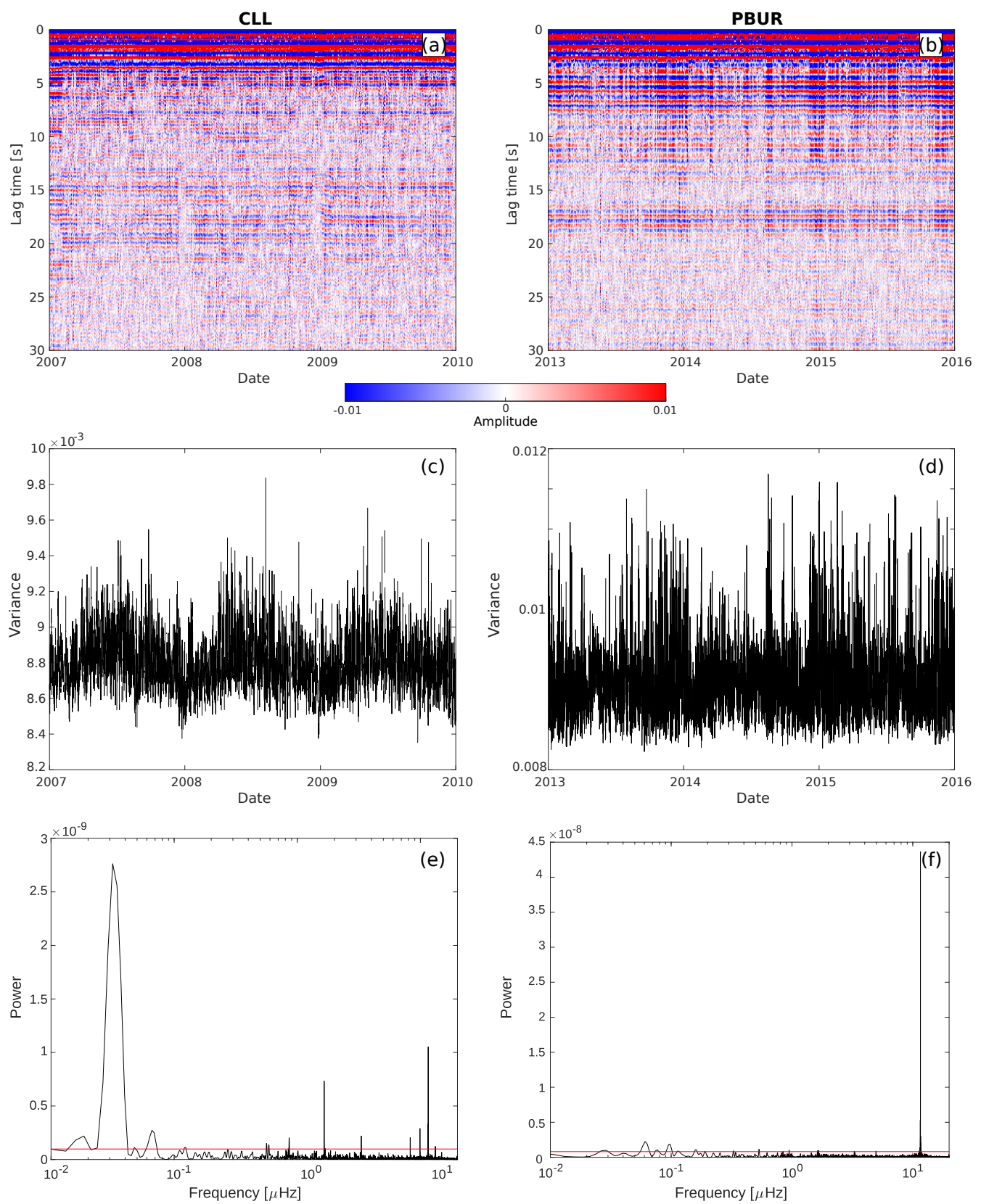

Figure 2.7: Top: Daily stacks of autocorrelations for stations CLL (a) and PBUR (b), filtered between 1 and $2 \mathrm{~Hz}$. (c,d) Variance over time. (e,f) Power spectra calculated with the LombScargle method from the variances of the single autocorrelations. The red line shows the $99 \%$ probability threshold of not being a random fluctuation. 


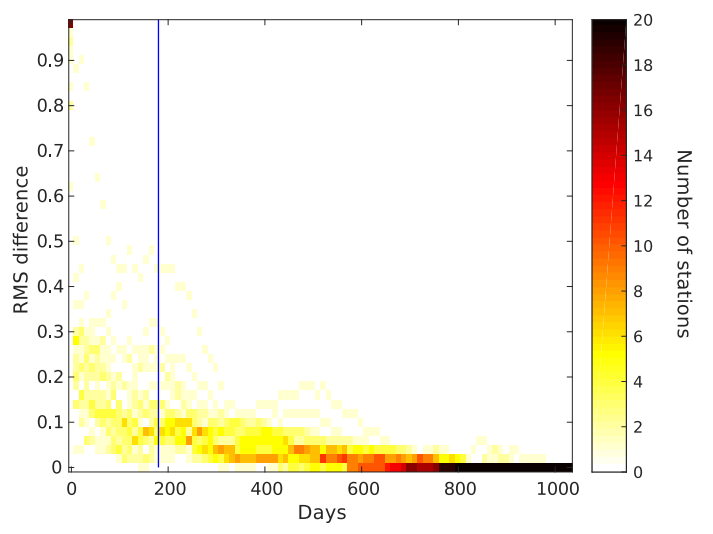

Figure 2.8: Data-density plot of the normalized RMS-value of difference between final stack result and continuous stacking of autocorrelations for different broad-band stations. Blue line indicates the 180 day cut-off.

intervals, and the final result after linear stacking of the entire time period is calculated similar to the approach by Chen et al. (2017). For reasons of inter-station comparability, it was decided to consider the entire lag time window of 0-30 s here, instead of a shorter time window, between the side lobes and later parts without significant signal. It would be difficult to set such a shorter time window equally applicable to all stations. The lower bound containing the first side lobes would vary for each station, and the amplitude does not vanish at later lag times for all stations, so there is no common end time. We compare the RMS-values to determine the time after which they do not decrease significantly any longer. Fig. 2.8 shows a data density plot of the normalized RMS-values for the broadband stations, with the RMS-values rapidly decreasing to lower levels. Some stations show a continuous decrease, for example, station UBBA. Other stations show more variable RMS-values with time. This coincides, for example, with distinct seasonal changes in autocorrelation amplitude. For all broad-band stations, the RMS-value permanently decreases to values below 0.1 after, on average, $250 \mathrm{~d}$ of data, i.e. roughly 2000 accepted $3 \mathrm{hr}$ time windows. Already after $180 \mathrm{~d}$, equivalent to 1440 accepted $3 \mathrm{hr}$ time windows, the mean RMS-value decreased to 0.14 and the median to 0.1 , which are sufficiently low to not change the resulting autocorrelation interpretation further significantly. We therefore set the threshold value of the minimum amount of data needed to obtain a stable result to $180 \mathrm{~d}$.

\subsubsection{Moho depths}

An overview of the results for the broad-band stations, filtered between 1 and $2 \mathrm{~Hz}$, along with the picked time for the arrival of the Moho-reflected $P$-wave (green, dashed) and with estimates based on Grad et al. (2009) (red) and Knapmeyer-Endrun et al. (2014) (blue) can be seen in Fig. 2.9. The grey boxes indicate the a priori time range calculated from a $5 \%$ velocity uncertainty and the depth uncertainty provided by Grad et al. (2009), which is postulated to contain the Moho arrival. The corresponding Moho depths can be found in Table 2.2 in comparison with estimates made by Grad et al. (2009) and KnapmeyerEndrun et al. (2014). For comparison, an overview of the autocorrelations obtained when 

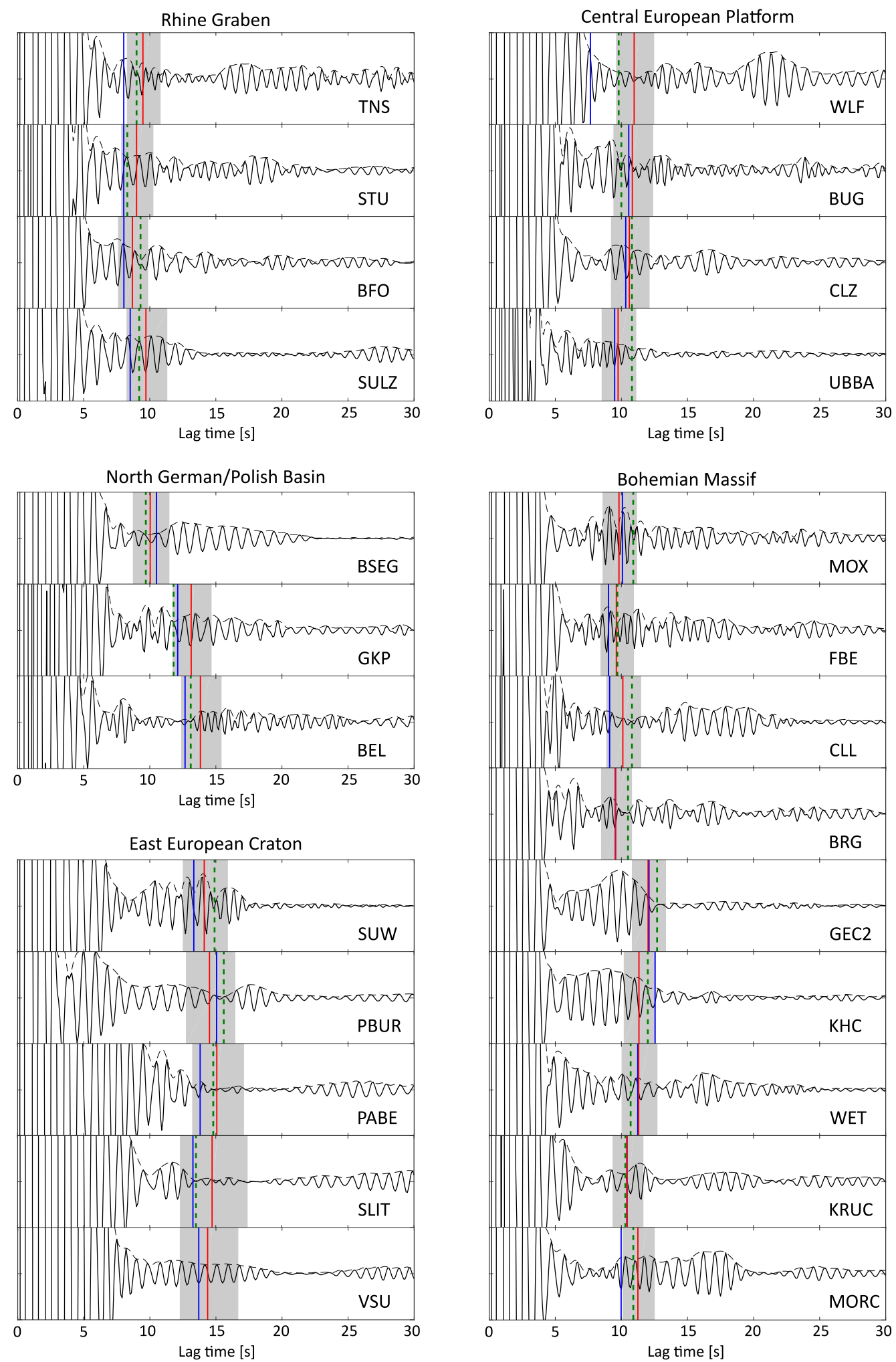

Figure 2.9: Autocorrelations, filtered between 1 and $2 \mathrm{~Hz}$, in black with envelopes (dashed, black) and results of automatically picked arrival times of Moho reflections (dashed,green), along with calculated times obtained from Knapmeyer-Endrun et al. (2014) (blue) and Grad et al. (2009) (red). Grey area indicates $5 \%$ velocity uncertainty combined with depth uncertainty provided by Grad et al. (2009). 
filtering between 2 and $4 \mathrm{~Hz}$ can be found in Fig. A2.18.

For the stations belonging to the Rhine Graben, the picked estimates compare very well with the values obtained by other studies, only showing small offsets. Stations BFO and SULZ, that are located in the southern part of the Rhine Graben, show similar results and the Moho can be identified as a decrease in amplitude which increases again afterwards. Station STU is located further north and further away from the Graben than the former stations. The Moho is not as clearly visible, but still indicated by a decrease in amplitude. At Station TNS in the north of the Rhine Graben the change in reflectivity is additionally associated with a change in frequency content.

For the North-German and Polish Basins the autocorrelations show very clear changes in reflectivity. Stations BSEG and BEL show a clear increase in amplitude at the Moho. For stations GKP and BEL in the Polish Basin, the reflectivity changes are also associated with a change in frequency. The automated picking algorithm has no problems in identifying these changes for all three stations and they match very precisely the arrival times found in other studies.

For the central European Platform, the change in reflectivity is clearly visible and easy to pick, but the picked reflectivity changes are slightly later (CLZ, UBBA) or earlier (BUG) than the previously estimated times. The exception is station WLF, where the previous estimates already differ by about $10 \mathrm{~km}$. Here, the pick is located between the two estimates. Stations BUG and UBBA additionally show a change in frequency content.

For stations located on the Bohemian Massif, the results vary. Stations GEC2 and KHC show similar results with an easily locatable change in reflectivity, marked by a profound decrease in amplitude. Stations BRG, CLL, FBE, MOX and WET also show clear changes in reflectivity, but of a different shape than the former stations. Whereas for stations GEC2 and KHC, the amplitude of the autocorrelations goes down to almost zero, the other stations show varying amplitudes after the reflectivity change. Station CLL and WET show a later increase in amplitude, after which the amplitude decreases. At station MOX, FBE and BRG, the amplitude does not decrease to zero in the lag time window considered. Except for stations KHC and WET, the picked times are slightly later than the previous estimates. Stations KRUC and MORC are located at the Eastern boundary of the Bohemian Massif and also show changes in reflectivity, which are marked by a small increase in amplitude. At around $20 \mathrm{~s}$, the amplitude decreases to zero, before it increases again at ca. $23 \mathrm{~s}$.

Stations PABE and SLIT located on the East European Craton show a similar result. A clear change in reflectivity is detectable by a strong decrease in amplitude. The picked times match the results of other studies. PBUR and SUW also show comparable waveforms with reflectivity changes in agreement with previous estimates. Station VSU does not show any change in reflectivity close to the estimates. The only changes visible are more than $5 \mathrm{~s}$ away in either direction. For this station, we also considered a different frequency band by filtering between 2 and $4 \mathrm{~Hz}$ (Sun \& Kennett 2017). A small change in reflectivity is now visible at the expected lag time (Figs 2.10a and b). Stations PABE, SLIT and VSU show much smaller amplitudes at the lag time window of the expected Moho reflectivity change than all of the other stations. The mean amplitude inside the a priori time window is about a factor of 10 smaller for stations SLIT and VSU and even a factor 100 for station PABE.

The mean difference between the determined depth value and the depth by Grad et al. 

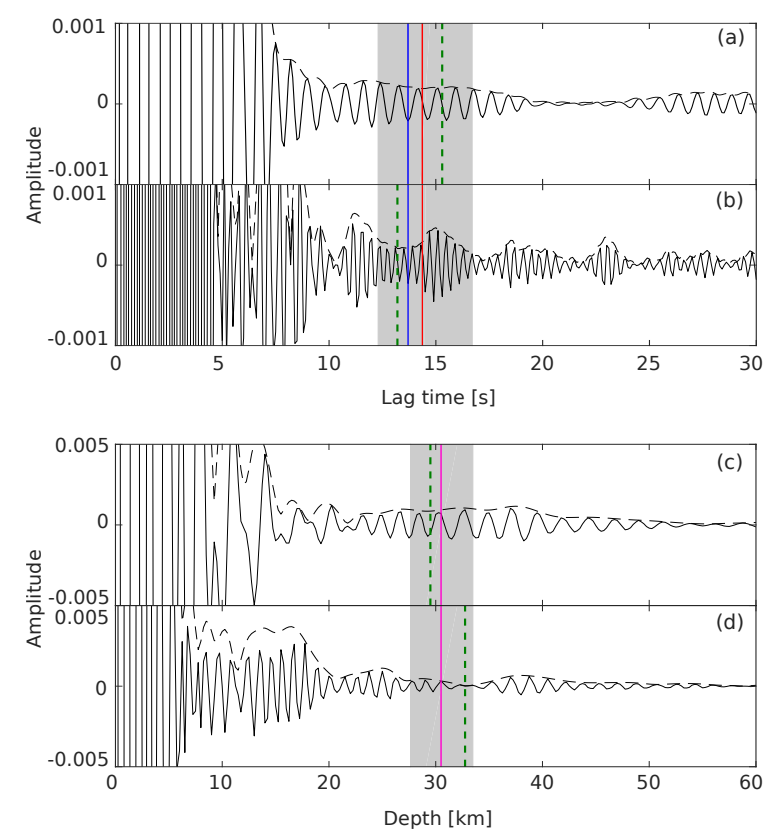

Figure 2.10: Autocorrelation of stations VSU and PB41 filtered between 1 and $2 \mathrm{~Hz}(\mathrm{a}, \mathrm{c})$ and 2 and $4 \mathrm{~Hz}(\mathrm{~b}, \mathrm{~d})$. Green dashed lines indicate picked changes in reflectivity. In (b), a small change in reflectivity is visible at around $14 \mathrm{~s}$. In (c), no clear change in reflectivity is visible around $11 \mathrm{~s}$, where the Moho reflection is expected to be. This change is however clearly recognizable in (d). Red and blue lines indicate calculated lag times of Moho reflected $P$-waves according to Grad et al. (2009) and Knapmeyer-Endrun et al. (2014). Purple line shows Moho depth taken from the velocity model provided by Grad et al. (2003). Grey area depicts uncertainty window of prior knowledge.

(2009) is $-0.2 \pm 2.5 \mathrm{~km}$. The maximum difference is $3.7 \mathrm{~km}$ for station PBUR, and the minimum difference is $-4.1 \mathrm{~km}$ for station GKP. The mean difference between the determined depth and the depth calculated from receiver functions (Knapmeyer-Endrun et al. 2014) is $1.6 \pm 2.4 \mathrm{~km}$. The maximum difference is $6.7 \mathrm{~km}$ for station WLF and $-2.4 \mathrm{~km}$ for station BSEG. When filtering between 2 and $4 \mathrm{~Hz}$ (Fig. A2.18), the mean difference in lag time compared to $1-2 \mathrm{~Hz}$ is $-0.2 \pm 1.0 \mathrm{~s}$, which converts to a mean difference in depth of $-0.7 \pm 3.2 \mathrm{~km}$.

\subsubsection{Profile P4}

After development and testing of the method with broad-band stations across Central Europe, it was applied to the mostly short-period and temporary stations of the PASSEQ network along profile line P4 (Fig. 2.1). The results obtained with the autocorrelations are compared with the subsurface model provided by seismic profiling. Lag times of the autocorrelations were converted into depth using the velocity model provided by Grad et al. (2003). Fig. 2.11 shows this velocity model overlain with picks of changes in reflectivity (blue and red symbols). 15 stations could not be used due to too little or faulty data, whereas 8 analysed stations did not show any reflectivity change within the a priori range in either frequency band.

The change to $P$-wave velocities greater $8 \mathrm{~km} \mathrm{~s}^{-1}$, representing the Moho, can again be 
Table 2.2: Overview of Moho depths determined for the broad-band stations. Moho depths of this study calculated from the picked lag times in the autocorrelation results in Fig. 2.9 and velocity values taken from Molinari \& Morelli (2011), Majdański (2012), Karousová et al. (2012). * indicates Moho depth picked in 2-4 Hz frequency band.

\begin{tabular}{lccc}
\hline Station & $\begin{array}{c}\text { Grad et al. }(2009) \\
\text { Moho depth }(\mathrm{km})\end{array}$ & $\begin{array}{c}\text { Knapmeyer-Endrun et al. (2014) } \\
\text { Moho depth }(\mathrm{km})\end{array}$ & $\begin{array}{c}\text { This study }(1-2 \mathrm{~Hz}) \\
\text { Moho depth }(\mathrm{km})\end{array}$ \\
\hline BEL & 43.0 & 39.4 & 40.7 \\
BFO & 25.7 & 23.8 & 27.5 \\
BRG & 29.1 & 29.0 & 31.9 \\
BSEG & 29.6 & 31.0 & 28.6 \\
BUG & 32.6 & 31.8 & 30.1 \\
CLL & 31.1 & 28.0 & 33.2 \\
CLZ & 31.8 & 31.0 & 32.4 \\
FBE & 29.4 & 27.6 & 29.6 \\
GEC2 & 36.6 & 36.8 & 38.7 \\
GKP & 40.1 & 37.0 & 36.0 \\
KHC & 37.1 & 41.1 & 39.3 \\
KRUC & 34.1 & 34.0 & 33.6 \\
MORC & 36.4 & 32.3 & 35.3 \\
MOX & 30.2 & 31.0 & 33.5 \\
PABE & 48.9 & 44.8 & 48.0 \\
PBUR & 45.8 & 47.5 & 49.5 \\
SLIT & 47.7 & 43.0 & 43.7 \\
STU & 26.9 & 24.0 & 24.8 \\
SULZ & 28.5 & 25.0 & 27.0 \\
SUW & 46.8 & 44.2 & 49.4 \\
TNS & 28.2 & 23.9 & 26.8 \\
UBBA & 29.6 & 28.8 & 32.8 \\
VSU & 47.2 & 45.0 & $33.4 *$ \\
WET & 34.2 & 34.0 & 30.7 \\
WLF & 34.4 & 24.0 & \\
\hline
\end{tabular}


recognized in the autocorrelations by looking for changes in reflectivity. These changes are indicated by blue symbols in Fig. 2.11, which were determined with the automated picking algorithm. Blue dots indicate picks made in the 1-2 Hz frequency band, blue stars represent picks made in the $2-4 \mathrm{~Hz}$ frequency band. For the majority of stations (24 out of 32 ), it is possible to pick the Moho depth in the 1-2 Hz frequency band. An overview of stations and used frequency bands can be found in Table A2.3. The increasing Moho depth from West to East can be traced in the depth values derived from autocorrelations. For some (8 out of 32) stations, the same situation occurs as for the broad-band station VSU (Figs 2.10a and b). No clear reflectivity change is visible within the depth range where it is expected, when filtering between 1 and $2 \mathrm{~Hz}$. When filtering between 2 and $4 \mathrm{~Hz}$, the results improve and it it possible to identify the Moho. An example of this effect can also be seen in Figs 2.10(c) and (d). For station PB41 at around profile kilometre 10, the result improves significantly when using a higher frequency band, and the picked depth matches well with the value given in the velocity model. The stations that are mostly affected by the different filters, lie below the Polish basin. For the aforementioned 8 out of the 23 unused stations, the change in frequency band did not lead to an improvement. Some of the Moho picks place the Moho at lower depth, but slightly more than half (17 out 32) of the picks place the Moho at greater depth. Especially between profile kilometres 400 to 700 the picks all indicate greater depths than the background model. The average difference between the determined depth and the Moho depicted by the velocity model is $0.29 \pm 1.6 \mathrm{~km}$ with a maximum difference of $1.75 \mathrm{~km}$ and a minimum difference of $-3 \mathrm{~km}$.

In addition to the Moho, an intracrustal interface can be identified, between 25 and $30 \mathrm{~km}$ depth. It is visible in the velocity model and can be traced with most stations (red symbols in Fig. 2.11). In the autocorrelations, it can mostly be identified as the first major change in reflectivity. An example for stations PN09 and PA73 is shown in Fig. 2.12. PN09 and PA73 are located at $c a .-100 \mathrm{~km}$ and $620 \mathrm{~km}$ profile length, respectively, and show two clear changes in reflectivity for the Moho and the intracrustal layer. Since the difference in depth between Moho and crustal layer is much larger for station PA73, the two picks are located further apart. For six stations, it is again only possible to identify the crustal layer in the 2-4 Hz frequency band. For determined depths and used frequency bands, refer to Table A2.3. These stations do not all coincide with the stations where the Moho can only be identified in the higher frequency band. The mean difference between the picked intracrustal depth and the depth visible in the velocity model is $-0.48 \pm 1.4 \mathrm{~km}$, with a maximum difference of $1.75 \mathrm{~km}$ and a minimum difference of $-3 \mathrm{~km}$. In general for both layers, the estimates vary between too shallow and too deep compared to the background model, but overall trace the layer boundaries very well all along the profile.

\subsection{Discussion}

Processing plays a significant role in the calculation of the autocorrelation and the analysis of the result. A small change in processing parameters can change whether a clear determination of Moho depth is possible or not. For ambient noise cross-correlations, it has been concluded that parameters have to be optimized for each data set individually (Fichtner et al. 2017). We try to develop a unified processing scheme that allows to evalu- 


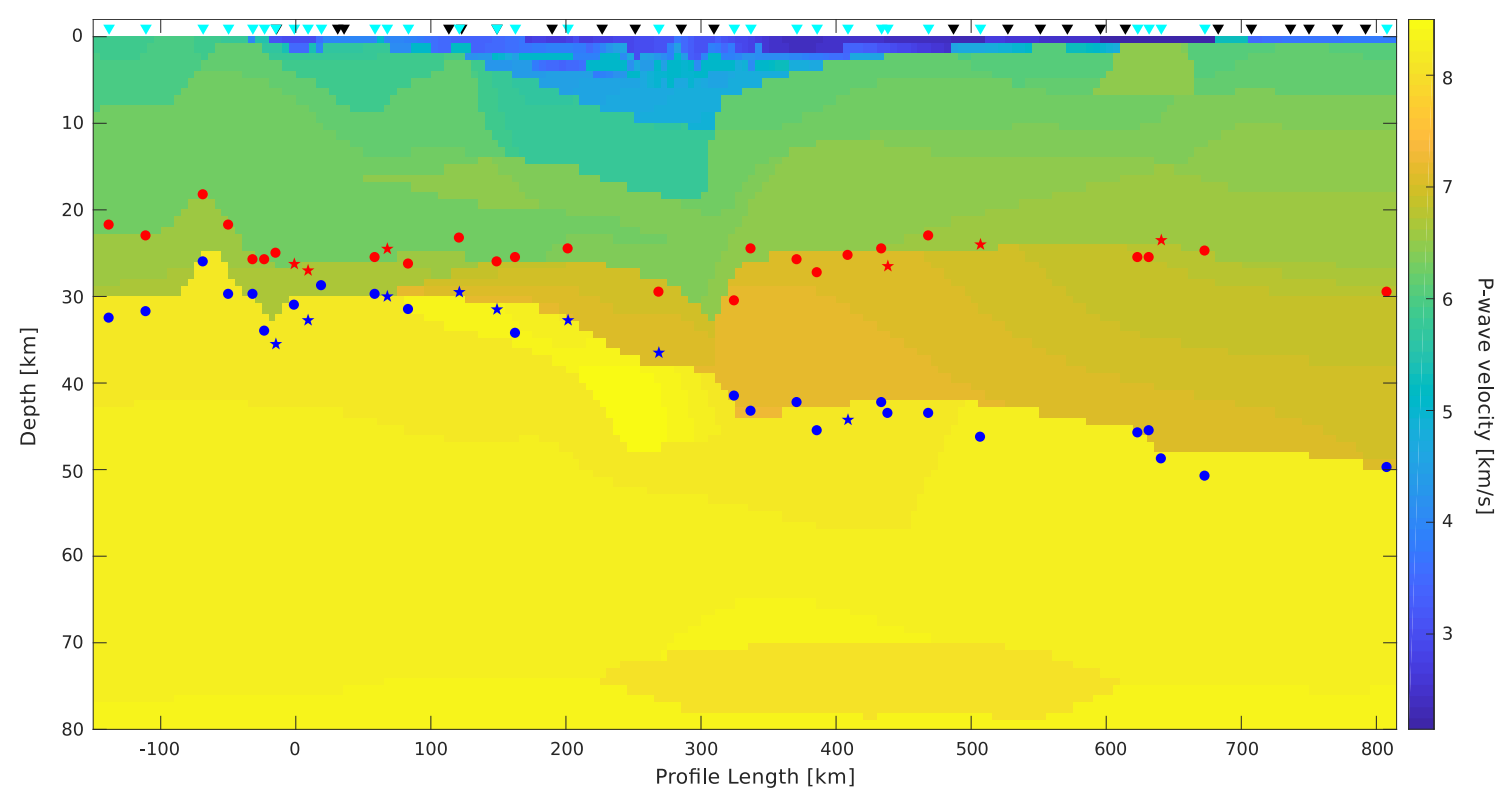

Figure 2.11: Changes in reflectivity derived from autocorrelation of PASSEQ stations along POLONAISE P4 profile from west to east, with the velocity model by Grad et al. (2003) used to convert lag time to depth in the background. Cyan inverted triangles: stations used; black inverted triangles: stations discarded due to faulty or too little data; blue dots: picked changes in reflectivity at Moho depth in the 1-2 Hz frequency band; blue stars: picked changes in reflectivity at Moho depth in 2-4 Hz frequency band; red dots: picked changes in reflectivity at intracrustal interface in 1-2 Hz frequency band; red stars: picked changes in reflectivity at intracrustal interface in $2-4 \mathrm{~Hz}$ frequency band.
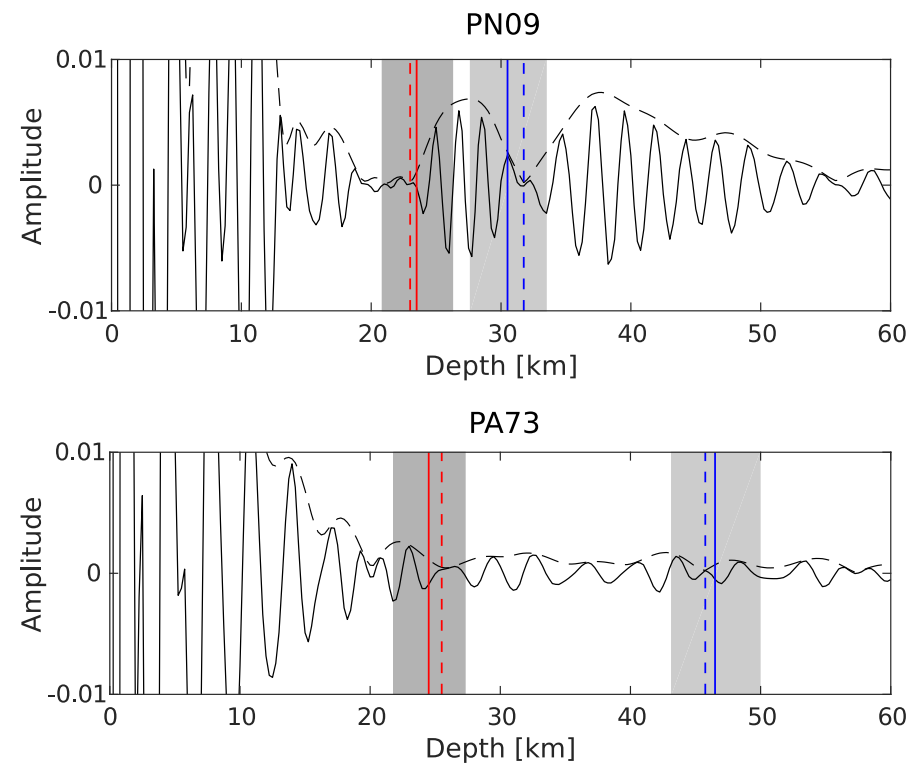

Figure 2.12: Example of intracrustal layer and Moho for stations PN09 and PA73 located at $c a$. $-100 \mathrm{~km}$ and $620 \mathrm{~km}$ profile length of profile P4 respectively, filtered between 1 and $2 \mathrm{~Hz}$. In red picked (dashed) and given (solid) intracrustal interface. Blue picked (dashed) and given (solid) Moho depth, as seen in profile P4. Picked values correspond to red and blue symbols in Fig. 2.11. Grey areas show $2 \mathrm{~km}$ depth and $3 \%$ velocity uncertainty range. 
ate autocorrelations in terms of crustal thickness over a broad range of crustal structures, over an area of roughly $1000 \mathrm{~km}^{2}$, and for permanent broad-band, as well as, temporary short-period stations.

The choice of the filtering parameters plays a very important role, as can be seen in Fig. 2.4. Low frequencies below $1 \mathrm{~Hz}$ are not suitable here, because sidelobes of the main peak extend up to more than $10 \mathrm{~s}$ lag time, masking crustal signals. When choosing the frequency band of 1-2 Hz, the crust-mantle interface can be recognized by a change in reflectivity in the signal, as the properties of the two layers vary. In the frequency band of $2-4 \mathrm{~Hz}$, the change in reflectivity is also visible, but it is not as pronounced for most broadband stations. In addition, changes in frequency content in the autocorrelation are not detectable in this frequency band (Fig. A2.18). It was therefore decided to filter between 1-2 Hz. For some, specifically short-period, stations no clear change in reflectivity is visible in the 1-2 Hz frequency band. For those stations, it is helpful to also filter between 2-4 Hz and compare the results (cf. Kennett 2015).

The picked lag times for the broad-band stations in the two frequency bands show a rather small mean difference of $-0.2 \mathrm{~s}$. Nevertheless, at some stations the absolute differences are larger than $1.5 \mathrm{~s}$. When inspecting the automatically picked lag times with respect to the waveform, the $2-4 \mathrm{~Hz}$ results do not always agree with the strongest change in reflectivity, though (e.g. stations STU, GKP, PBUR, SUW and MOX, Fig. A2.18). The higher frequency content causes a rougher envelope, which does not encompass complete wave packages without intermediate minima, making it difficult to detect large-scale changes in reflectivity. For the $1-2 \mathrm{~Hz}$ results, the picked lag times generally match visible reflectivity changes. In most cases, these picked lag times additionally agree with the reflectivity changes visible after filtering between 2 and $4 \mathrm{~Hz}$. Exceptions are stations TNS, UBBA and SLIT, where the reflectivity changes in the two different frequency bands do not match. Therefore, we show the obtained Moho depths for the 1-2 Hz frequency band in Table 2.2, except for station VSU, where filtering between 1 and $2 \mathrm{~Hz}$ does not give a meaningful result.

The application of a high pass filter before the actual processing significantly improves the results, because it eliminates sidelobes of the central peak. It allows the depiction of shallower discontinuities, which would otherwise be covered by the sidelobes.

By smoothing the spectrum of the data prior to calculation of the autocorrelation, the quality of the results is greatly improved as ringing and other artefacts caused by very narrow-band signals are removed. This type of industrial signal is abundant across densely populated Central Europe (Bokelmann \& Baisch 1999), and it is therefore important to minimize its influence on the processing result. Smoothing the spectrum alters the data significantly, as it changes amplitudes in the spectrum, but it provides the opportunity to include stations that would otherwise have to be excluded (Fig. 2.2). After applying this processing step, the autocorrelations show clearer changes in reflectivity and also for stations not influenced by ringing (Fig. 2.3).

The application of the time-frequency domain phase-weighted stack improves the final autocorrelation most when no spectral smoothing is applied, as it enhances coherent phases. When applying spectral smoothing, the improvement is not as significant, as can be seen in Fig. 2.3. Incoherent signals arriving at later lag times are suppressed, but the type of stacking does not change the overall shape of the stack considerably. This allows us to compare linearly stacked autocorrelations for each day with respect to their temporal 
properties (Figs 2.7a and b).

The temporal behaviour of the autocorrelations shows different characteristics. Some stations show higher variances during the summer, whereas others show higher variances during the winter. The stations showing higher variances during the winter are located closer to the coast, such as, for example, BSEG, PBUR, SLIT. Since we are considering the frequency band between 1-2 Hz, which contains cultural as well as natural noise (Bonnefoy-Claudet et al. 2006), the higher variance for stations close to the sea, can be related to more and higher waves hitting the shore in the winter. The oceanic microseismicity also exhibits higher amplitudes in winter (Aster et al. 2008), for both primary microseisms mainly between $0.05-0.08 \mathrm{~Hz}$ and secondary microseisms mainly between 0.1-0.16 Hz (Longuet-Higgins 1950, Hasselmann 1963, Schimmel et al. 2011a). However, microseisms are not included in the frequency band considered and can therefore not be responsible for the observed increase in variance. Stations showing higher variances in the summer could be influenced by cultural noise, such as, for example, construction work being more active in the summer. Overall it becomes apparent that most stations are influenced by cultural noise, since especially weekly variations, i.e. between work days and weekends, cannot be explained by natural causes. Likewise daily variations are easily explained by cultural noise, because during the nighttime activity related to traffic, machinery and factories is reduced. Another indication of cultural noise is the observation of narrow-band machine signals. Overall, we can conclude that one of the main noise sources used here is cultural noise and the noise wave-field is therefore non-stationary with time.

The developed method is spatially consistent and only shows very small variations for the different stations of the GERESS array. These variations can arise due to small local subsurface variations and the direction of noise sources. The main features, for example, the change in reflectivity at the Moho depth, are visible for all stations. When comparing the correlation coefficient between all stations with respect to the distance between the stations, the correlation decreases with distance from 0.999 to 0.986 . This is expected, since neighbouring stations should show more similar results.

In addition to spatial consistency, the method also gives consistent results for different kinds of seismometers. When removing the instrument response and applying a high pass filter to the data, the results of the collocated broad-band and short-period seismometer are identical. The consistency among different seismometers makes it possible to apply the method to whatever data are available. It is important to compensate for the instrument response, when directly comparing different seismometer types. All other stations are analysed independently, and compensating for the instrument response is not necessary in these cases, as the main features of the autocorrelation are not influenced (see Fig. A2.16). For stations of the GERESS array, the considered time window length is reduced to $1 \mathrm{hr}$, in order to have more time windows available. The difference in the stacked autocorrelations when varying between $1 \mathrm{hr}$ and $3 \mathrm{hr}$ time windows, is small (see Fig. A2.17), which allows reduction of the time window to $1 \mathrm{hr}$ in order to make the inclusion of stations with more data gaps possible.

The determination of the Moho depth poses one of the main challenges in autocorrelation analysis. The identification of the Moho is often based on prior information (Kennett 2015, Sun \& Kennett 2017), consistency among neighbouring stations (Tibuleac \& von Seggern 2012, Kennett et al. 2015, Taylor et al. 2016) or forward modelling (Oren \& 
Nowack 2017). In this study, the Moho depth is picked as the base of the crustal reflector, indicated by a change in reflectivity. It is known from prior studies (e.g. Artemieva \& Meissner 2012, and references therein), that most of west-central Europe is characterized by a strongly reflective lower crust. This implies a distinct change in reflectivity at the Moho as well as between the upper and lower crust, which facilitates identification of the intracrustal interface (Fig. 2.12) although velocity contrasts are smaller west of the TESZ. The changes in reflectivity are automatically determined as maxima of the weighted second derivative of the envelope of the obtained autocorrelation. These maxima correspond to points of the curve, where the slope of the envelope changes and coincide with changes in reflectivity. The weighting of the second derivative ensures that later changes in reflectivity show amplitudes in accordance with their real amplitudes. With prior information on $P$-wave velocity and estimates of Moho depth, the highest local maximum inside the predicted time window and matching clearly visible changes in reflectivity is chosen as the base of the crustal reflector. Instead of defining a target window, a priori information could alternatively be formulated probabilistically in a Bayesian approach. Automating the picking process makes the method consistent, repeatable and independent of the analyst.

The potential of the autocorrelation method to determine subsurface structure can clearly be seen when looking at the mean differences and standard deviations obtained from the broad-band stations and the stations along profile P4. The deviations are in good agreement with the deviations of $\pm 4 \mathrm{~km}$ determined by Grad et al. (2016), when comparing the European Moho depth map (Grad et al. 2009) to crustal thickness from a more detailed 3-D seismic model of Poland. The mean differences of the broad-band stations and the PASSEQ stations along the profile to prior estimates are about the same. The difference for the receiver functions results (Knapmeyer-Endrun et al. 2014) is slightly larger than for the depth provided by Grad et al. (2009). The receiver functions tend to result in smaller Moho depths than estimated here from autocorrelations, though the same average crustal velocities were used. However, the two methods look at different phases, with a different spatial sampling. Receiver functions use $P$-to- $S$ conversions and their multiples, sampling a Fresnel zone of 10-50 km around the station for the direct conversion and up to $70 \mathrm{~km}$ for the multiples. Accordingly they may average over spatial variations in Moho depth differently than the autocorrelations, with an additional bias caused by sources mainly located to the Northeast. Besides, a gradual Moho transition with a highly reflective lower crust might influence different data sets in different ways (Hrubcová \& Geissler 2009). When calculating the autocorrelations and assuming vertically incident $P$-waves, the variable slownesses of the wave field contributing to the stacked result is not accounted for (Gorbatov et al. 2013). It is assumed that the reflections occur directly below the station, but in reality, the region of stationary phase extends to several 10s of $\mathrm{km}$ around the station for sources coming from below (Oren \& Nowack 2017). These considerations could explain the later arrival of phases and resulting larger Moho depth estimates. Furthermore, some of the stations are located below the NorthGerman and Polish basins. This may lead to additional scattering and reverberations of the waveforms, diffusing the result.

The general trend of increasing Moho depth from West to East can clearly be followed along profile $\mathrm{P} 4$ with the reflectivity changes of the single autocorrelations. In addition to the Moho, an additional interface can be observed by the first major changes in reflectivity 


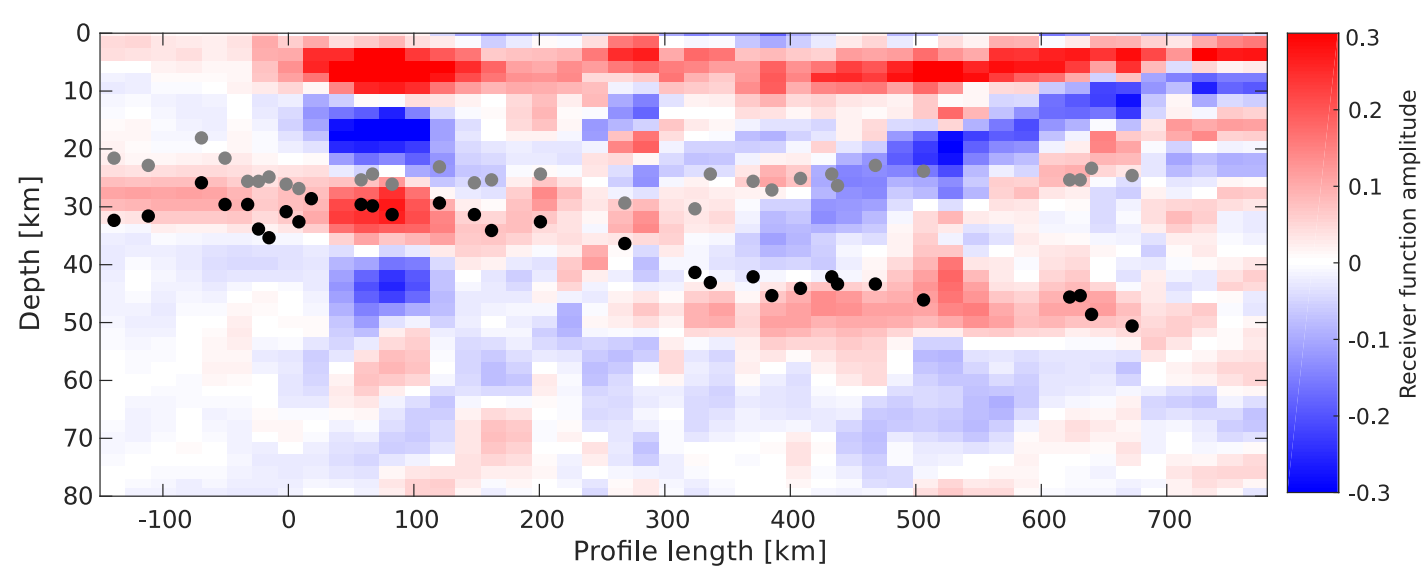

Figure 2.13: Comparison of receiver function result obtained by Knapmeyer-Endrun et al. (2014) with Moho and crustal layer picks, obtained in this study (grey dots: crustal layer; black dots: Moho).

between 20 and $30 \mathrm{~km}$ and followed across the profile from West to East, proving that the method is suitable to detect interfaces at varying depths. The depth range of the Moho regarded here is comparable to estimates of Martian crustal thickness calculated from gravity measurements (Neumann et al. 2004, Wieczorek \& Zuber 2004), making autocorrelation analysis a suitable tool for the InSight mission.

The Central European Moho has been extensively studied by Knapmeyer-Endrun et al. (2014) using receiver functions. When comparing the obtain results of Moho depth and crustal layer with the receiver function results, it is clearly visible that the results match (Fig. 2.13). The thickening of the crust from West to East is observable and the Moho picks align with the features visible in the receiver function image. The intracrustal discontinuity is not observable in the receiver function result. In the western part of the profile the discontinuity cannot be distinguished from the Moho within the resolution of the migrated image. The autocorrelations exhibit a higher resolution due to the higher frequencies considered and can resolve the two discontinuities. In the eastern part, a possible signal from the intracrustal discontinuity is overlaid by sediment multiples in the receiver function image. However, an intracrustal discontinuity around $20 \mathrm{~km}$ depth was observed in receiver functions for individual stations east of the TESZ (Knapmeyer-Endrun et al. 2014). Slight differences in Moho depth can again be explained by the different velocity models employed. In the migration of the receiver functions, the 1-D IASP91 model (Kennett \& Engdahl 1991) was used, whereas for the autocorrelations, the detailed 2-D velocity model obtain by seismic profiling was applied, making the depth estimates more accurate.

The broad-band stations located across different geologic settings allow us to compare the obtained autocorrelations with respect to their subsurface. The overall lower amplitude of the stations PABE, SLIT and VSU located on the East European Craton coincides with the lower reflectivity in the lower crust in Eastern Europe compared to the Variscides (Grad et al. 2002, Artemieva \& Meissner 2012). The velocity contrast between the lower crust and upper mantle is additionally significantly lower in the craton, due to the mafic lower crustal layer (Grad et al. 2003).

Stations BSEG and BEL located in basins show a decrease in amplitude, before it in- 
creases again after the Moho pick. At station GKP, decreases and increases in amplitude are lower, but nevertheless visible. This behaviour is also detectable for stations PBUR and SUW and to a lesser extent for PABE. All of these stations are located in areas with low-velocity sediment loads of varying thicknesses (Molinari \& Morelli 2011). Specifically for station SUW, a strong velocity contrast between sedimentary cover and crust is detectable (Wilde-Piórko et al. 2017). These low-velocity layers show a significant impact on the waveforms due to reverberations caused by multiple reflections inside the sedimentary layer (Knapmeyer-Endrun et al. 2014). These reverberations could explain the increase in amplitude after the Moho, for example, by interference of multiple reflections in the sediment layer with reflections from the Moho and other impedance contrasts (Nishitsuji et al. 2016).

At stations GEC2 and KHC, the amplitude decreases drastically after the Moho pick. Other stations show additional features, for example, later increases in amplitude (e.g. PABE, SLIT, WLF, VSU, SULZ), changes in frequency content (e.g. TNS, CLL, FBE, BEL) or a combination of both. For 16 out of the 25 stations, we observe another clear reflectivity change at later lag times within the first $30 \mathrm{~s}$, accompanied by an increase in amplitude (Fig. 2.9). To investigate whether this reflectivity change coincides with the lithosphere-asthenosphere boundary (LAB), as seen in autocorrelations by Kennett (2015), we pick the coinciding maximum in the second derivative, after filtering between 1 and $2 \mathrm{~Hz}$. The lag times of the picks are converted into depth assuming the crustal velocity provided by the velocity models and a uniform velocity of $8.04 \mathrm{~km} \mathrm{~s}^{-1}$ for the mantle based on IASP91 (Kennett \& Engdahl 1991). The resulting depths are plotted as circles in Fig. 2.14 on a background map of LAB depth west of the TESZ and the depth of a midlithosphere discontinuity (MLD) east of the TESZ (Knapmeyer-Endrun et al. 2017b). The depths determined here show good agreement with the depths determined from $P$ - and $S$ receiver functions by Knapmeyer-Endrun et al. (2017b), with a mean deviation of $-6.2 \mathrm{~km}$ (Fig. 2.14). The two stations with a large discrepancy between the two measurements are located close to strong gradients in mapped LAB or MLD depth. Some minor deviations can be expected, due to the different velocity models used to convert traveltime to depth (IASP91 for receiver functions, local crustal velocities for autocorrelations). For stations located at the Rhine Graben and the Eger Rift, the shallower LAB, at 50-65 km and $c a .70 \mathrm{~km}$ depth, respectively, can clearly be identified and picked. The thin lithosphere extends across a broader region around the Upper Rhine Graben, supporting the receiver function results. For the other tectonic regimes, deeper reflectivity changes can not be observed for all stations. Some of the stations located East of the Trans-European Suture Zone show a very clear increase in amplitude at later lag times, corresponding to 83 to $93 \mathrm{~km}$ depth. This coincides with the increase in reflectivity at similar depth observed by Nielsen et al. (2002) along a peaceful nuclear explosion seismic profile across Eurasia and the MLD mapped by Knapmeyer-Endrun et al. (2017b). All other stations, indicated by white dots in Fig. 2.14, do not show a later increase in amplitude again, or like station CLZ show a continuous increase and decrease in amplitude, with no clear reflectivity change visible. For the majority of broad-band stations, it appears to be possible to identify deeper discontinuities such as the lithosphere-asthenosphere boundary, which is in accordance with Kennett (2015). 


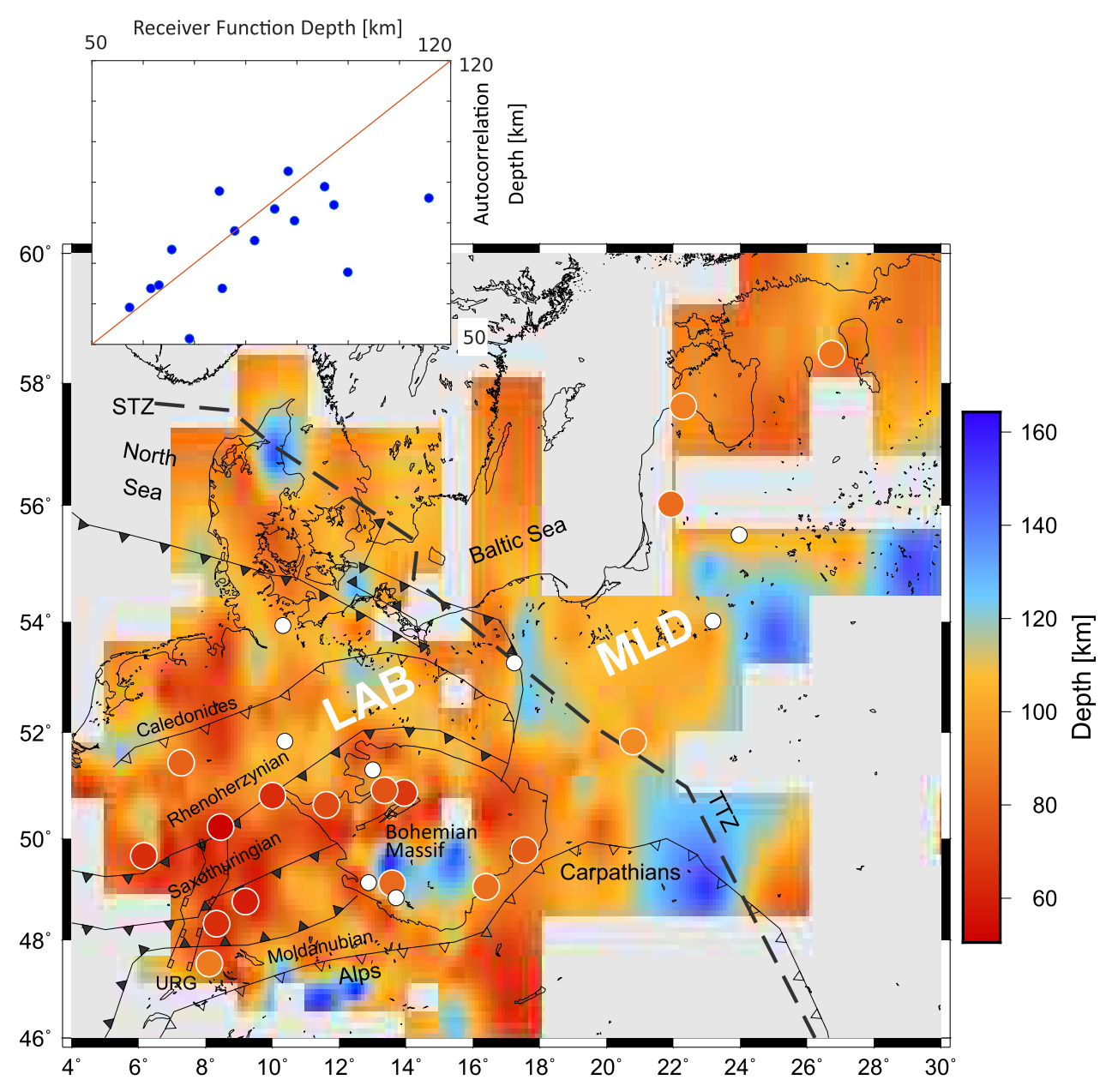

Figure 2.14: Map of lithosphere-asthenosphere boundary depth west of the TESZ and midlithosphere depth east of the TESZ from Knapmeyer-Endrun et al. (2017b). The broad-band station locations and determined depths, based on autocorrelations filtered between 1-2 Hz, are indicated by circles. White circles indicate stations without later increase in autocorrelation amplitude. The inset on top shows the depth obtained by receiver functions versus the depth determined from the autocorrelations. The red line has a slope of one. For labels refer to Fig. 2.1.

\subsection{Conclusions}

We show here that with the appropriate processing the calculation of autocorrelations provides a means to identify strong subsurface changes in velocity, composition or reflectivity, such as the Moho. By using a phase autocorrelation algorithm and filtering between 1-2 Hz, we demonstrate that no strong energy sources such as earthquakes or microseims are needed to successfully retrieve Moho depth from autocorrelations. The application of a spectral smoothing algorithm to the amplitude spectrum of the data allows the inclusion of stations which are strongly influenced by very narrow-band industrial signals within the frequency range of interest. The described processing of high pass filtering, spectral smoothing, phase autocorrelation, time-frequency phase weighted stacking, and band pass filtering leads to spatially consistent results and is applicable to different types of seismometers.

By incorporating prior information in the form of a time or depth window, Moho 
depths and additional discontinuities can automatically be detected as changes in reflectivity in the autocorrelations. The application of the method to stations across Europe reproduces the thickening of the Moho from West to East across the TESZ. Our approach shows that the method of ambient noise autocorrelation can provide important information of strong interfaces in the subsurface, without the need for multiple stations, or even multiple components at a station. It is a valuable tool when only moderate amounts of data (about six months) are available, or station coverage is low, as will be the case, for example, for the InSight mission to Mars in 2018, and it offers a flexible way to incorporate prior information. 


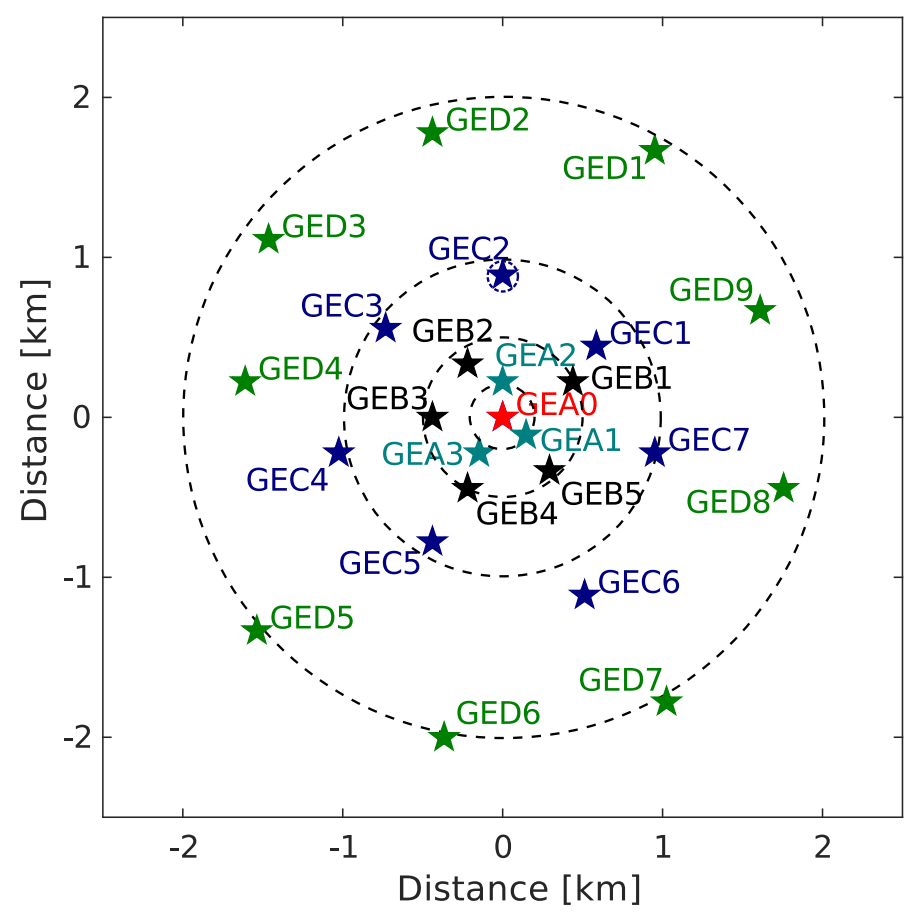

Figure A2.15: Layout of GERESS array. Central point at station GEA0. Stations with the same colours belong to the same ring. Collocated broad-band and short-period station GEC2 is indicated by a circle.

\section{A2.A Appendix}

Fig. A2.16 compares the final stacked results for broad-band station GEC2 without removal of the instrument response in (a) and after removal of the instrument response prior to calculation of the autocorrelation and stacking in (b). The overall shape and characteristics are the same, whereas some small details at later lag times (e.g. between 20-25 s) are influenced slightly. Fig. A2.17 shows the results for broad-band stations GEC2 after calculating the autocorrelations for different time window lengths of $1 \mathrm{hr}, 3 \mathrm{hr}$ and $6 \mathrm{hr}$. Some small differences are visible, but the overall shape and characteristics, specifically regarding the change in reflectivity related to the Moho at $12.7 \mathrm{~s}$, are detectable in all three results. 


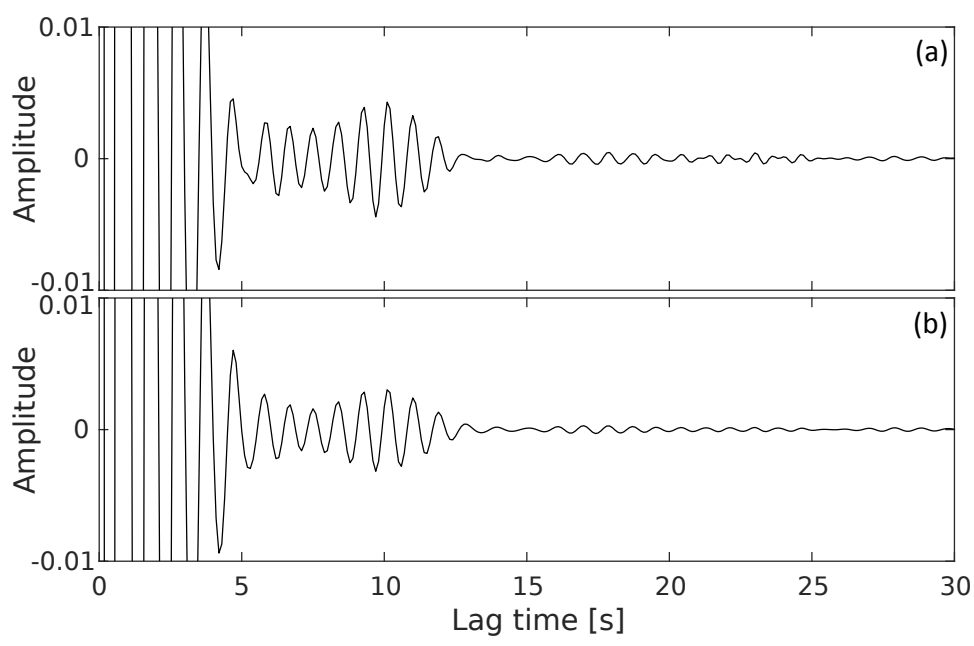

Figure A2.16: Comparison of tf-PWS stacked autocorrelation result for broad-band station GEC2 for window length of $1 \mathrm{hr}$, without removal of the instrument response in (a). (b): same processing as in (a), but a restitution filter to remove the instrument response is included before any other processing steps.

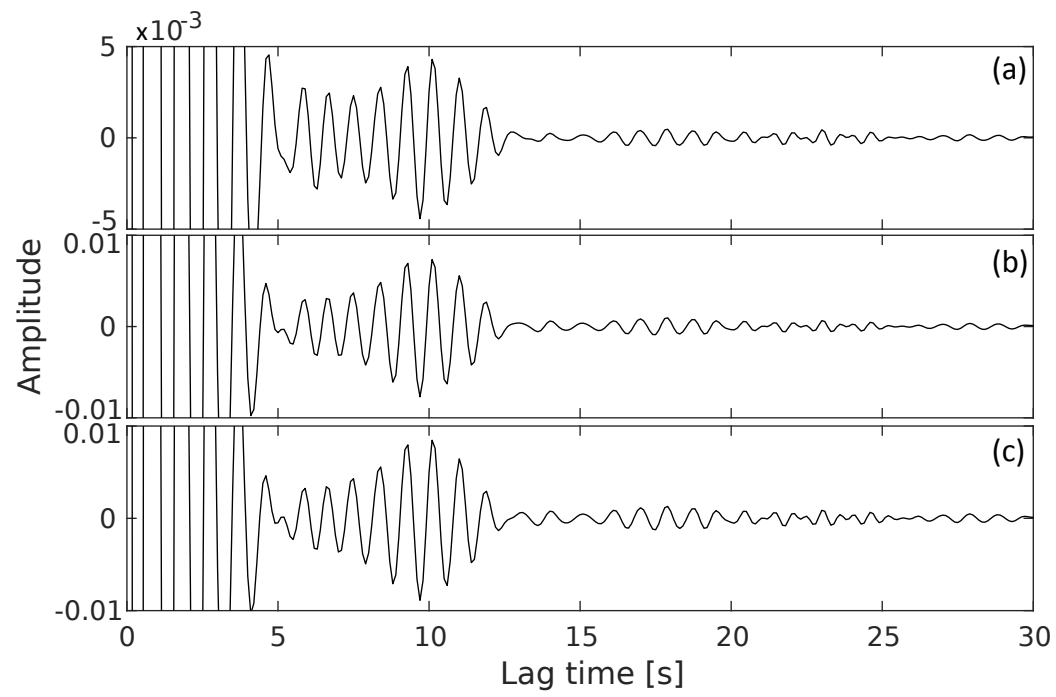

Figure A2.17: Comparison of stacked autocorrelation results for broad-band station GEC2 with window length of $1 \mathrm{hr}$ (a), $3 \mathrm{hr}$ (b) and $6 \mathrm{hr}$ (c) used for the calculation of the autocorrelations. The number of stacked windows is: 22747 windows for $1 \mathrm{hr}$ length $(=948 \mathrm{~d}), 5571$ windows for $3 \mathrm{hr}$ length (=696 d) and 1762 windows for $6 \mathrm{hr}$ length (= $440 \mathrm{~d})$. 

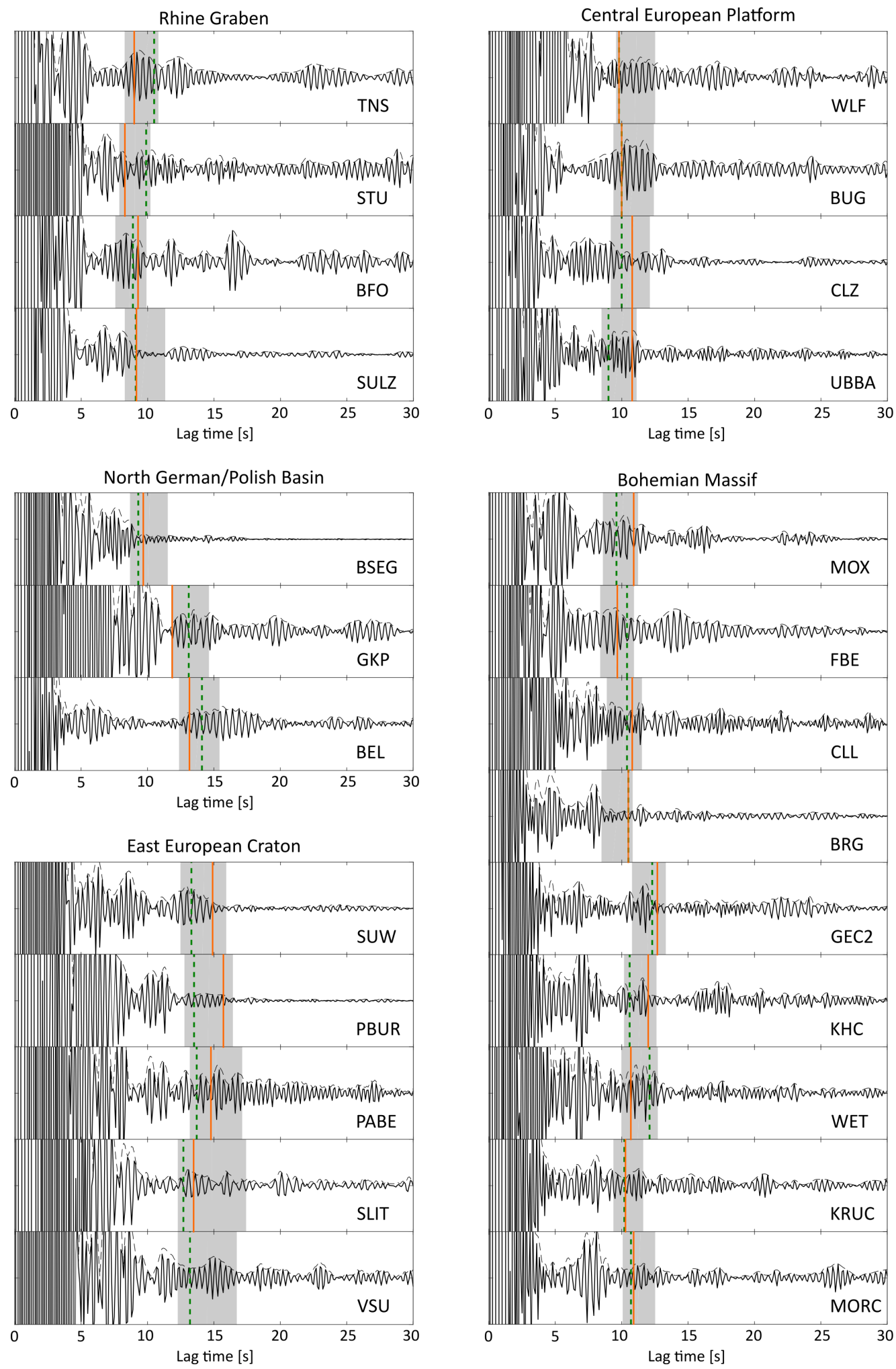

Figure A2.18: Autocorrelations filtered between 2-4 Hz in black with envelopes (dashed, black) and results of automatically picked arrival times of Moho reflections (dashed, green), along with picks made in the 1-2 Hz frequency band in orange. Grey area indicates $5 \%$ velocity uncertainty combined with depth uncertainty provided by Grad et al. (2009). 
Table A2.3: Overview of intracrustal layer and Moho depths determined for the stations along Profile P4. * indicates depths picked in the $2-4 \mathrm{~Hz}$ frequency band. ${ }^{\dagger}$ indicates stations with only broad-band or a majority of broad-band data for PASSEQ stations with instrument changes.

\begin{tabular}{|c|c|c|c|c|}
\hline \multirow[b]{2}{*}{ Station } & \multicolumn{2}{|c|}{ Intracrustal layer [ km] } & \multicolumn{2}{|c|}{ Moho depth [ km] } \\
\hline & Profile P4 & This study & Profile P4 & This study \\
\hline $\mathrm{CLL}^{\dagger}$ & 23.5 & 21.75 & 30.5 & 32.5 \\
\hline PN09 & 23.5 & 23 & 30.5 & 31.75 \\
\hline PN11 & 18.5 & 18.25 & 25.5 & 26 \\
\hline PB19 & 21.5 & 21.75 & 28.5 & 29.75 \\
\hline PA20 $0^{\dagger}$ & 25.5 & 25.75 & 31.5 & 29.75 \\
\hline PB22 & 26.5 & 25.75 & 32.5 & 34 \\
\hline $\mathrm{PB}_{23 \mathrm{~B}^{\dagger}}$ & 26.5 & 25 & 32.5 & $35.5^{*}$ \\
\hline PB25 & 27.5 & $24 *$ & 30.5 & 31 \\
\hline PB41 & 26.5 & $27 *$ & 30.5 & $32.75^{*}$ \\
\hline PB42 & 26.5 & - & 30.5 & 28.75 \\
\hline PB43 & 26.5 & 25.5 & 30.5 & 29.75 \\
\hline PB44 & 26.5 & $24.5^{*}$ & 30.5 & $30 *$ \\
\hline PA64 ${ }^{\dagger}$ & 25.5 & 26.25 & 30.5 & 31.5 \\
\hline PB46B & 25.5 & 23.25 & 30.5 & $29.5 *$ \\
\hline PA65 $5^{\dagger}$ & 26.5 & 26 & 31.5 & $31.5^{*}$ \\
\hline PB47 & 26.5 & 25.5 & 31.5 & 34.25 \\
\hline $\mathrm{PA} 66^{\dagger}$ & 26.5 & 24.5 & 33.5 & $32.75^{*}$ \\
\hline PA $67^{\dagger}$ & 28.5 & 29.5 & 38.5 & $36.5^{*}$ \\
\hline PA68 ${ }^{\dagger}$ & 28.5 & 30.5 & 44.5 & 41.5 \\
\hline PB53 & 26.5 & 24.5 & 44.5 & 43.25 \\
\hline PB54 & 25.5 & 25.75 & 43.5 & 42.25 \\
\hline PA69 ${ }^{\dagger}$ & 25.5 & 27.25 & 43.5 & 45.5 \\
\hline PB55 & 25.5 & 25.25 & 42.5 & $44.25^{*}$ \\
\hline PB56 & 25.5 & 24.5 & 42.5 & 43.25 \\
\hline PA70 ${ }^{\dagger}$ & 25.5 & $26.5^{*}$ & 42.5 & 43.5 \\
\hline PB57 & 25.5 & 23 & 42.5 & 43.5 \\
\hline PA $71^{\dagger}$ & 25.5 & $24^{*}$ & 43.5 & 46.25 \\
\hline $\mathrm{PA} 73^{\dagger}$ & 24.5 & 25 & 46.5 & 45.75 \\
\hline $\mathrm{SUW}^{\dagger}$ & 24.5 & 25.5 & 47.5 & 45.5 \\
\hline PB81 & 24.5 & $23.5 *$ & 48.5 & 48.75 \\
\hline PB82 & 25.5 & 24.75 & 48.5 & 50.75 \\
\hline PB87 & 27.5 & 29.5 & 50.5 & 49.75 \\
\hline
\end{tabular}




\title{
3 Crustal thickness from horizontal component auto- and cross-correlations
}

\author{
Optimism is the faith that leads to \\ achievement.
}

Helen Keller

The contents of this chapter have been submitted to Geophysical Journal International as "Crustal thickness from horizontal component seismic noise auto- and crosscorrelations for stations in Central and Eastern Europe" in 2018. The work, writing and creation of figures were carried out by myself, under the supervision of B. KnapmeyerEndrun, from the Max Planck Institute for Solar System Research.

\subsection{Summary}

Extracting Moho-reflected $P$-waves from vertical component seismic noise to determine crustal thickness has become a common method over the last years. In this study, we image the reflectivity below various stations across Central and Eastern Europe and extract Moho-reflected $S$-waves using horizontal component correlations. In addition to horizontal component autocorrelations, we include single-station cross-correlations in the process of obtaining crustal thickness estimates from seismic noise for the first time. For each station, a lag time range for the reflectivity change associated with the Moho is obtained, and converted lag times are converted to depth to give a crustal thickness range. In addition, we calculate $v_{\mathrm{P}} / v_{\mathrm{S}}$-ratios from the picked reflectivity changes on the vertical and horizontal component correlations.

On average 8.5 months of data are needed for the autocorrelations to obtain stable results. For the cross-correlations, on average $1.3 \mathrm{yr}$ of data are required to reach stability. The obtained Moho depth ranges compare well with results determined with vertical component autocorrelations, receiver functions, and provided in the European Moho depth map. The $v_{\mathrm{P}} / v_{\mathrm{S}}$-ratios show a large variability and do not give reliable results. In addition to identifying reflectivity changes in $S$-waves on the horizontal component correlations, it is also possible to observe these reflectivity changes on vertical component correlations, as well as $P$-wave reflectivity changes on the horizontal component correlations, 
for some stations. Furthermore, we can detect $P$ - to $S$ - or $S$ - to $P$-converted phases on cross-correlations of mixed horizontal and vertical components. From this we conclude that the incidence of the waves reaching the surface is not generally vertical, leading to errors when converting lag time to depth and in the calculation of the $v_{\mathrm{P}} / v_{\mathrm{S}}-$ ratios. Lag time differences between the Moho arrivals on the two horizontal component autocorrelations can be interpreted in terms of azimuthal anisotropy in the crust.

\subsection{Introduction}

The extraction of Moho-reflected body waves from seismic noise autocorrelations has been successfully applied a number of times over the last few years. Already in 1968, Claerbout came to the conclusion that the autocorrelation of transmitted waves is equal to the reflection response of these waves. This approach can be used to determine the depths of discontinuities from ambient noise correlations. A number of studies have looked at vertical component data to identify Moho-reflected $P$-waves from the corresponding autocorrelations (e.g. Tibuleac \& von Seggern 2012, Gorbatov et al. 2013, Kennett et al. 2015, Taylor et al. 2016, Oren \& Nowack 2017). Kennett (2015) and Becker $\&$ Knapmeyer-Endrun (2018) additionally detected $P$-waves reflected at the lithosphereasthenosphere transition. Here, we look at horizontal components of seismograms and calculate auto- and single station cross-correlations from seismic noise to extract Mohoreflected $S$-waves.

Up to date, the only study which included horizontal component noise data in the calculation of autocorrelations for the extraction of Moho-reflected body waves was conducted by Tibuleac \& von Seggern (2012). In this study, autocorrelations for the three components of stations of the USArray in Nevada were calculated and prominent arrivals across all stations and autocorrelations were detected. These arrivals were interpreted as Moho-reflected $P$ - and $S$-waves. From this and from a polarization analysis it was concluded that the $P$-waves are non-vertically polarized and the $S$-waves non-horizontally, which explains the detection of both wave types on all autocorrelations. In addition, Tibuleac \& von Seggern (2012) calculated $v_{\mathrm{P}} / v_{\mathrm{S}}$-ratios, which agree well with results obtained from receiver functions for some stations and disagree for others. Oren \& Nowack (2017) also observed both Moho-reflected $P$ - and $S$-waves on vertical component noise autocorrelations, but did not consider horizontal components. Phạm \& Tkalčić (2017) and Sun $\&$ Kennett (2016) used the $P$-wave coda of teleseismic events to calculate the $P$ - and $S$-wave reflectivity from radial and vertical component autocorrelations, and to extract reflected body waves. Sun \& Kennett (2016) additionally calculated single-station crosscorrelations of radial and vertical component data, which were compared with receiver functions.

To our knowledge, seismic noise single-station cross-correlations have not been considered in the extraction of body waves so far. Other studies calculated autocorrelations and single-station cross-correlations to estimate the Green's function and analyse velocity variations, related to, for example, earthquakes, seasonal changes in humidity, or volcanic eruptions, by detecting changes in the coda of the correlations (e.g. Sens-Schönfelder \& Wegler 2006, Hobiger et al. 2014, De Plaen et al. 2016). In this study we extract the crustal thickness from seismic noise by detecting $S$-wave reflectivity changes and compare 
the obtained thicknesses to results determined from vertical component autocorrelations (Becker \& Knapmeyer-Endrun 2018), receiver functions (Knapmeyer-Endrun et al. 2014), and the European Moho depth map (Grad et al. 2009) based on a combination of methods, for example seismic and gravity measurements. In addition, we verify the obtained results by comparing them to reflectivity changes visible in vertical-horizontal component mixed cross-correlations. The processing used in Becker \& Knapmeyer-Endrun (2018) was adapted from the calculation of autocorrelations to the calculation of cross-correlations.

With the help of not only the vertical component autocorrelation, but also horizontal component auto- and mixed component cross-correlations, it is possible to extract further information about the subsurface, such as $v_{\mathrm{P}} / v_{\mathrm{S}}$-ratios, and to cross-check and verify results, even when data from only a single station is available.

\subsection{Data and study area}

In this study we use data from 25 three-component broad-band stations (STS-2, $120 \mathrm{~s}$ eigenperiod) located across Central and Eastern Europe in different geologic settings. The vertical component data of these stations have been analysed in Becker \& KnapmeyerEndrun (2018). Here we focus on the horizontal components and use the same three years of data. The different geologic settings covered here are characterized by varying Moho depths and overall subsurface properties. According to Grad et al. (2009), Moho depth varies between $25.7 \mathrm{~km}$ and $48.9 \mathrm{~km}$ below the stations. Three stations (BEL, BSEG, GKP) are located in basins (North German and Polish Basin), which are filled with low velocity sediments $\left(\mathrm{v}_{\mathrm{p}}<5 \mathrm{~km} \mathrm{~s}^{-1}\right)$ of up to $10 \mathrm{~km}$ thickness (Grad et al. 2003, Lamarche \& Scheck-Wenderoth 2005, Scheck-Wenderoth \& Lamarche 2005). Four stations (BFO, STU, SULZ, TNS) are located in the Rhine Graben, where Moho (Brun et al. 1992) and asthenosphere (Glahn \& Granet 1992) are shallow due to rifting. Another four stations (BUG, CLZ, UBBA, WLF) are situated on the Central European Platform, where crustal thickness is very homogeneous (Artemieva \& Meissner 2012, Meier et al. 2016). The East European Craton is sampled by five stations (PABE, PBUR, SLIT, SUW, VSU). It has been stable since at least $1.45 \mathrm{Ga}$ and is now overlain by a young, thin sedimentary cover (Bogdanova et al. 2006). The Teisseyre-Tornquist-Zone (TTZ) and the SorgenfreiTornquist-Zone (STZ) form the boundary between the East European Craton and Phanerozoic Central Europe. The remaining nine stations are located in the Bohemian Massif, on the outcropping crystalline basement of the Variscan orogeny (Matte et al. 1990). Fig. 3.1 shows an overview of the stations and their tectonic settings.

The crustal thickness of Central and Eastern Europe has extensively been studied with receiver functions amongst others by Wilde-Piórko et al. (2005), Geissler et al. (2008), Wilde-Piórko et al. (2010), Grad \& Tiira (2012) and Knapmeyer-Endrun et al. (2014). Grad et al. (2009) combined results from different methods (e.g. gravity and seismic measurements) to form a European Moho depth map. The Moho depths obtained by Grad et al. (2009) and Knapmeyer-Endrun et al. (2014) are used as references throughout this work.

The GERESS array located at the border between Germany and the Czech Republic consists of 25 short-period seismometers and one broad-band station collocated with one of the short-period seismometers. The stations are arranged in four concentric rings with 


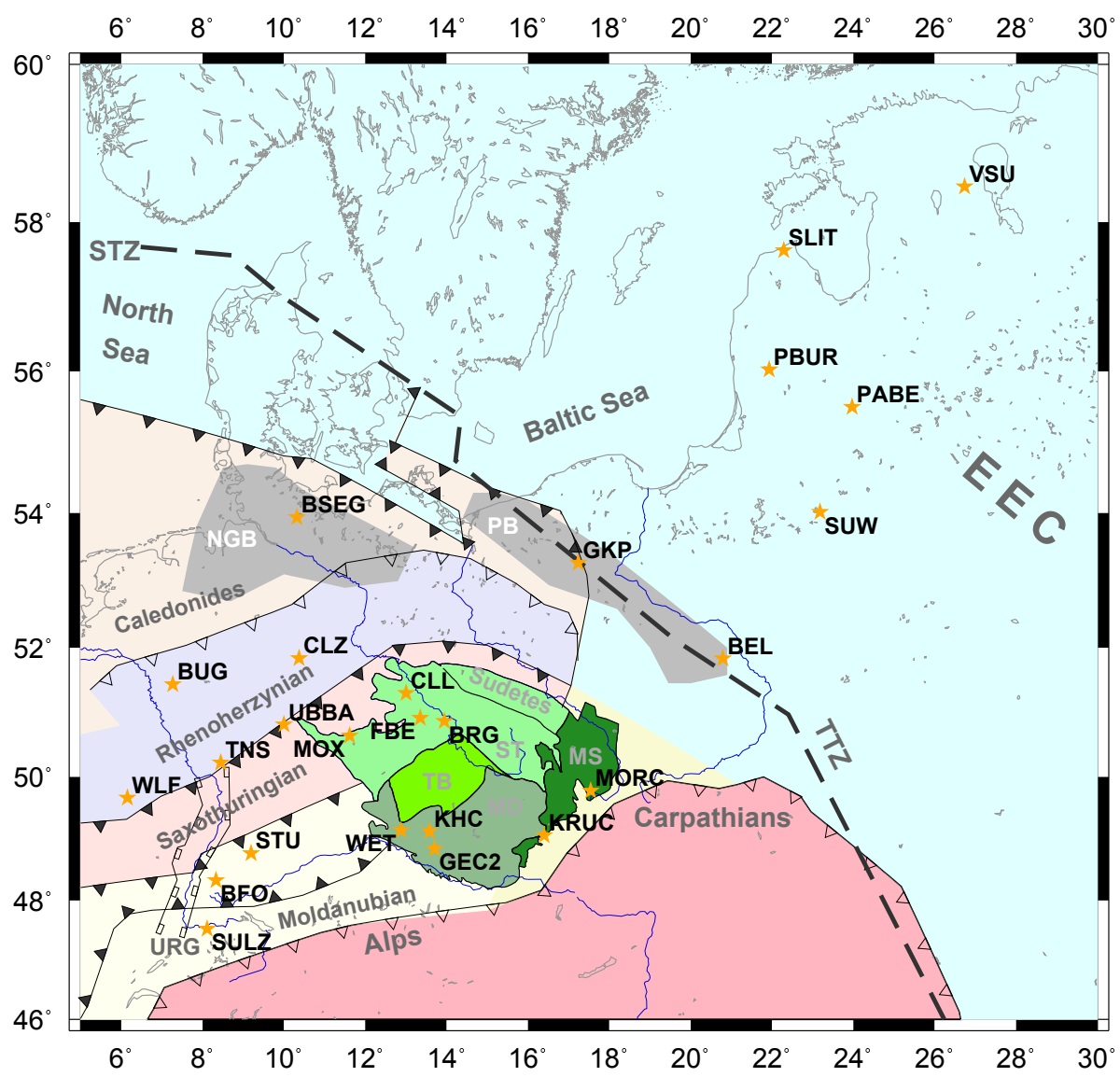

Figure 3.1: Overview of the tectonic setting of the study area (modified after Knapmeyer-Endrun et al. (2014)). Orange stars indicate the broad-band stations used. Labels: EEC: East European Craton; MD: Moldanubian of Bohemian Massif; MS: Moravo-Silesian of Bohemian Massif; NGB: North German Basin; PB: Polish Basin; ST: Saxothuringian of Bohemian Massif; STZ: Sorgenfrei-Tornquist Zone; TB: Tepla-Barrandian of Bohemian Massif; TTZ: TeisseyreTornquist-Zone; URG: Upper Rhine Graben

a maximum diameter of $4 \mathrm{~km}$. Four of the short-period stations and the broad-band station (GEC2) are equipped with three-components sensors. Distances between the threecomponent stations vary between $0.7 \mathrm{~km}$ and $3.4 \mathrm{~km}$, with a mean distance of $2.2 \mathrm{~km}$. These stations are used to compare results for horizontal components of stations in close vicinity to each other.

\subsection{Method}

\subsubsection{Processing and calculation of auto- and cross-correlations}

Autocorrelations for horizontal component data are calculated in the same way as explained in Becker \& Knapmeyer-Endrun (2018). The data are down-sampled to $10 \mathrm{~Hz}$ and cut into $3 \mathrm{hr}$ time windows. Time windows with data gaps or spikes are eliminated. A high-pass filter with a corner frequency of $0.5 \mathrm{~Hz}$ is applied and the mean and trend are removed for each time window. Spectral smoothing is applied to remove frequency 
spikes, the phase autocorrelations and the time-frequency domain phase weighted stack (tf-PWS) are calculated for each component according to Schimmel et al. (2011b). The advantage of the phase-autocorrelation is that it makes use of the instantaneous phase of a signal and is therefore an amplitude-unbiased method. It enhances coherent parts of the signal, whereas incoherent parts are suppressed. The use of the tf-PWS further amplifies this. The tf-PWS is again based on the similarity of the instantaneous phase, this time of the single correlations, and down-weights incoherent parts of the linear stack. This weighted stack is then used for the analysis and determination of Moho depth.

In addition to autocorrelations, for each station cross-correlations between the two horizontal components and between each horizontal component and the vertical component are calculated. The general processing is very similar to that of the autocorrelations. Instead of the phase autocorrelations, the phase cross-correlations according to Schimmel et al. (2011b) are calculated between two components. This gives two autocorrelation results for the horizontal components and another two results from the horizontal component cross-correlations, which should in theory lead to the same Moho depths as the same waves are considered. The four cross-correlations between horizontal and vertical components provide additional information, which can be used for further analysis and evaluation of the data.

For the stations of the GERESS-array, the instrument response is removed as a first processing step to ensure comparability between the stations. We additionally reduce the time window length to $1 \mathrm{hr}$ to include more time windows, since for some stations no or only very few $3 \mathrm{hr}$ time windows are available. Other than that, the processing is the same as for the broad-band stations.

\subsubsection{Determination of $v_{\mathrm{P}} / v_{\mathrm{S}}$-ratio and crustal thickness}

All auto- and cross-correlation results are filtered between 1 and $2 \mathrm{~Hz}$ and the second derivatives of their envelopes are calculated. Maxima in the second derivative represent changes in slope in the envelope, which concur with changes in reflectivity. We detect the Moho by a change in reflectivity visible in the correlations, as done, for example, by Kennett et al. (2015), Kennett (2015) and Becker \& Knapmeyer-Endrun (2018), and as opposed to picking amplitude maxima indicating the boundary (e.g. Tibuleac \& von Seggern (2012) and Gorbatov et al. (2013)). Picking the Moho as the base of the crustal reflection is of advantage when the Moho is gradational or not a strong, clear reflector (Kennett et al. 2015). In order to pick the reflectivity change coinciding with the crustmantle boundary, an uncertainty window, including prior information on the expected Moho depth (Grad et al. 2009) and crustal $S$-wave velocity (Molinari \& Morelli 2011), is defined. Using a velocity uncertainty of $\pm 5 \%$ and Moho depth uncertainties according to Grad et al. (2009), expected Moho depth and crustal $S$-wave velocity are converted into lag time to define a target window by the simple linear relation between two-way travel time (lag time), velocity and depth, assuming vertical incidence.

All four second derivatives obtained from the horizontal component auto- and crosscorrelations and the correlations themselves are compared and the reflectivity change, i.e. the maximum of the second derivative, visible in all correlations inside the uncertainty window is picked as the one coinciding with the Moho depth. This process is aided by plotting the four second derivatives obtained for each station next to each other as black 


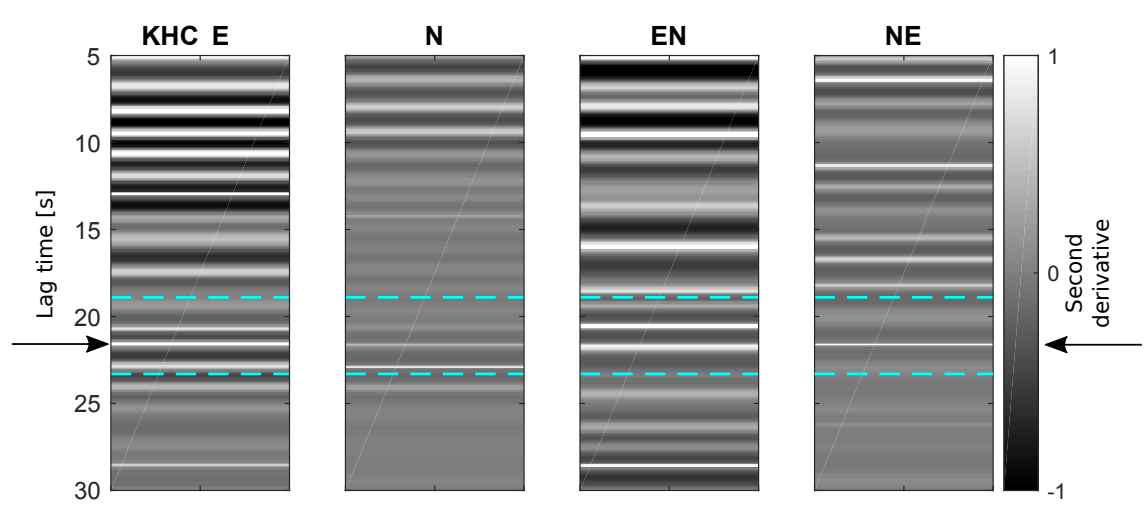

Figure 3.2: Second derivatives of horizontal correlations of station KHC filtered between 1 and $2 \mathrm{~Hz}$. Grey shading of image-plot depends on the amplitude of the second derivative. Cyan lines indicate the calculated target window. Arrows point to reflectivity change visible in all correlations.

and white image-plots, similar to Kennett et al. (2015), in order to detect maxima present in all correlations. Fig. 3.2 shows an example of these plots for station KHC. The cyan lines indicate the target window and the arrows highlight the reflectivity change visible in all correlations within the window. Not all stations show a unique maximum within the uncertainty window. Those reflectivity changes correlated across all correlations within the target uncertainty window are picked and the smallest and largest picked lag times form the upper and lower bounds of reflectivity change, as a result window. The velocity information provided in the EPcrust model (Molinari \& Morelli 2011) is used to convert the picked lag times into depths, resulting in a depth range for the Moho. For the autocorrelations calculated from vertical component data, we only derived a single depth, as only a single trace is available, but no further assessment of uncertainty could be made. Here, multiple correlations are considered, which results in the estimate of a depth range, including an uncertainty estimate.

Average crustal $v_{\mathrm{P}} / v_{\mathrm{S}}$-ratios are calculated by taking the lag times and dividing them by the picked lag times obtained from the vertical component autocorrelations (Becker \& Knapmeyer-Endrun 2018). This results into a range for the $v_{\mathrm{P}} / v_{\mathrm{S}}-$ ratio, which can then be studied further.

When a horizontal component correlation does not show any change in reflectivity at all, or no change in reflectivity that coincides with the other correlations is detectable, the correlation is filtered between 2 and $4 \mathrm{~Hz}$ instead and compared to the other results again. If a matching reflectivity change can be identified now, it is picked in this frequency band and $v_{\mathrm{P}} / v_{\mathrm{S}}$-ratio and Moho depth are calculated accordingly. If no matching reflectivity change is visible within the target window in either frequency band, this correlation is ignored and only the remaining correlations are considered.

The cross-correlations of horizontal and vertical component data can be used to check the assumption that the seismic waves arrive at the surface with vertical incidence. Assuming that the seismic waves do not impinge on the Moho exactly from vertically below, we also expect to see arrivals of phases converted from $P$-waves to $S$-waves and vice versa. The reflectivity changes associated with converted waves would be detectable on 
the cross-correlations of the mixed component data. If the waves arrive with vertical incidence instead, no converted phases would be created.

\subsection{Results}

\subsubsection{Temporal properties}

The analysis of the single autocorrelations before stacking allows us to draw conclusions about the nature of the noise sources used and the temporal properties of the correlations. The variances calculated for each $3 \mathrm{hr}$ autocorrelation for the lag time window of 0-30 s show specific characteristics. This time window is chosen, because it is expected to contain all crustal phases. In general, the two horizontal components of each station autocorrelations show the same temporal behaviour. The majority of stations shows higher amplitudes of the variance during the northern hemisphere summer time (17 out of 25), whereas five stations (CLZ, SLIT, SUW, TNS, VSU) show higher amplitudes during the northern hemisphere winter (Fig. 3.3). Two stations (WLF, PABE) do not show any regular variations. One station (SULZ) shows varying amplitudes with a period of roughly four months. Four stations (BSEG, CLZ, PBUR, TNS) exhibit different temporal behaviour, depending on the component of data used. The vertical component autocorrelations of stations BSEG, CLZ and PBUR show higher amplitudes in the winter, whereas the horizontal component autocorrelations show higher amplitudes in the summer. At station TNS, the opposite behaviour can be observed.

We then calculate the Lomb-Scargle-periodograms (Lomb 1976, Scargle 1982) to analyse the frequency behaviour of the changes in variance for each station. The LombScargle method is used to account for the data being unequally spaced and containing data gaps. The variances are clearly influenced by the day-night cycle, as all stations show a clear peak at the frequency corresponding to one day. Some stations are additionally influenced by weekly and/or yearly variations and show peaks at the corresponding frequencies and harmonics thereof. Station SULZ also clearly shows daily and weekly variations, but instead of the yearly dependence, it shows peaks at the frequency of four and two months, which is consistent with the above mentioned amplitude periodicity. Examples for stations with higher variances during summer and winter time, along with the corresponding Lomb-Scargle-periodograms can be found in Fig. 3.3. The peak visible in all four periodograms at roughly $3 \times 10^{-2} \mu \mathrm{Hz}$ is equal to the frequency of one year. The peak at roughly $11 \mu \mathrm{Hz}$ equals the frequency of one day. The small peak visible in the periodogram of the East-West component of station BEL at roughly $2 \mu \mathrm{Hz}$ is equivalent to one week.

In order to determine the amount of data needed to obtain a stable result, we continuously linearly stack the $3 \mathrm{hr}$ autocorrelations and calculate the root-mean-square (RMS) difference to the final linear stack of all autocorrelations, similar to Chen et al. (2017). The data density plots for the two horizontal components are shown in Figs 3.4(a) and (b). We assume that once the RMS-difference of all stations permanently decreases to below 0.1 , the correlations are stable and do not change significantly by adding more traces. This median value of the RMS-differences of all stations permanently decreases below 0.1 after $259 \mathrm{~d}$ for both components. This is equal to $20723 \mathrm{hr}$ time window autocor- 

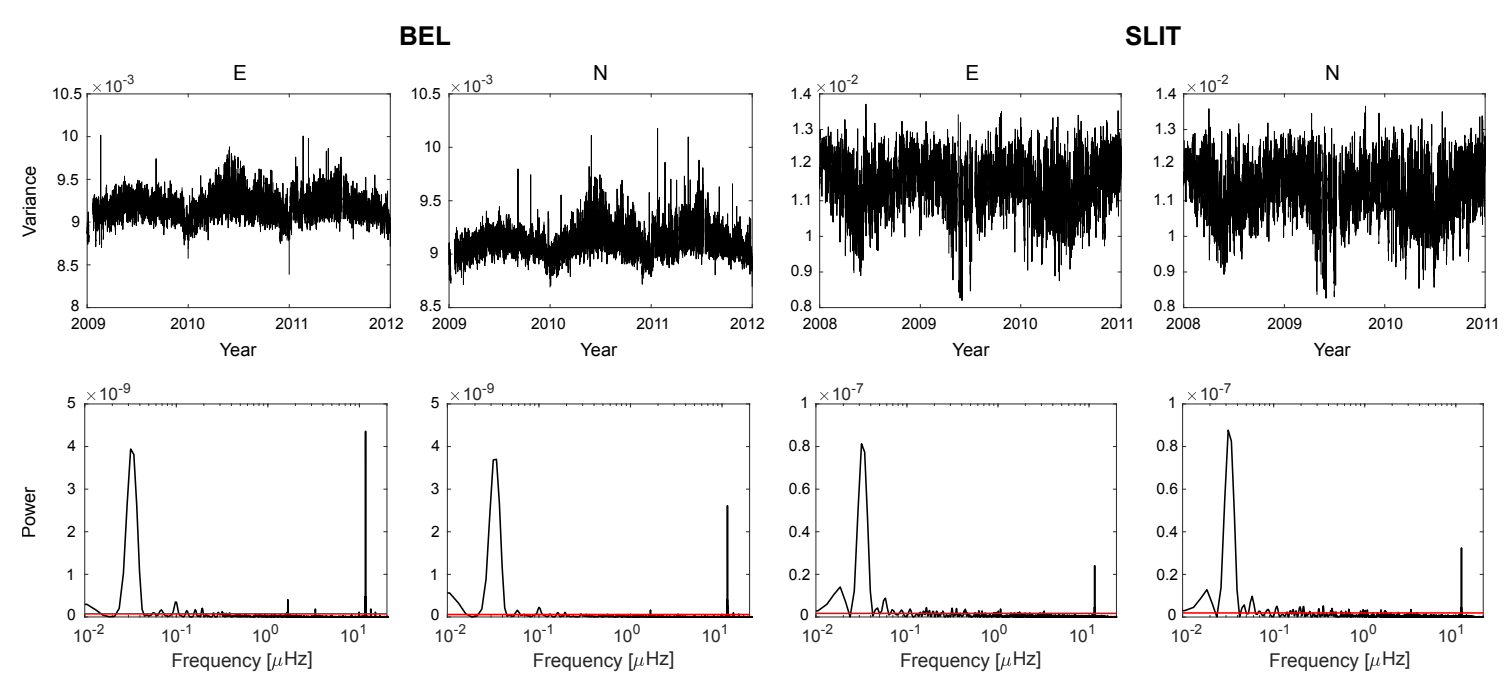

Figure 3.3: Temporal properties of autocorrelations from stations BEL and SLIT for the two horizontal components. Top row: Calculated variances for 0-30 s lag time. Bottom row: Corresponding Lomb-Scargle-Periodograms. Red line: $99 \%$ threshold of the frequency peak being a random fluctuation.

relations. Some stations, for example FBE, SUW and VSU, reach a RMS-difference of 0.1 only after twice as much time, though. In general, after approximately 8.5 months the autocorrelation results for both components are stable and do not change much any more afterwards.

The cross-correlations exhibit a different behaviour. The overall number of correlations stacked in the final stack is comparable to the number of autocorrelations, on average the number of cross-correlations is only smaller by $6 \%$. Nevertheless, the stacked crosscorrelations converge much slower to a final, stable result. When cross-correlating the two horizontal components, the median RMS-difference permanently decreases to below 0.1 only after $488 \mathrm{~d}$ for one of the cross-correlations and after 506 days for the other one (Figs 3.4c and d). Approximately twice as many cross-correlations of horizontal components, as opposed to autocorrelations, are needed to obtain a stable result. The cross-correlations of horizontal with vertical components converge a bit faster. The median RMS-differences have decreased to 0.1 after 421 and $447 \mathrm{~d}$ for the East-West with vertical component cross-correlation. For the North-South component cross-correlated with the vertical component, the median RMS-difference decreases to 0.1 after 328 and $345 \mathrm{~d}$.

\subsubsection{Spatial consistency}

Five stations of the GERESS-array are three component seismic stations. One of these stations is station GEC2, which is in addition a broad-band station. Three of the threecomponent stations are located on the outermost ring (GED1, GED4 and GED7), whereas station GEA2 is located on the inner ring, and GEC2 roughly in the middle. Stations GEA2 and GEC2 are located the closest together with a distance of $0.7 \mathrm{~km}$. Stations GED1 and GED7 are located furthest from each other with a distance of $3.4 \mathrm{~km}$. Fig. 2.6 shows the autocorrelation results of the horizontal component data for those stations. The 

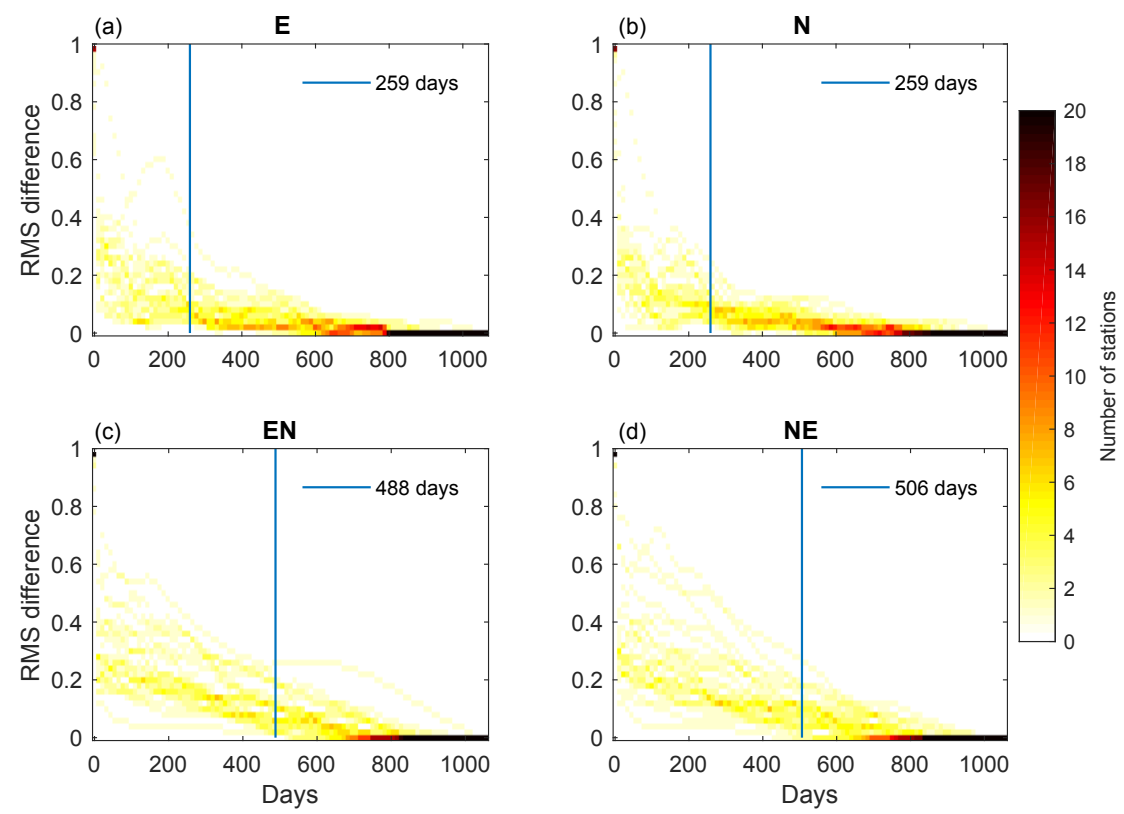

Figure 3.4: Data density plots of the root-mean-square (RMS) difference of the final linear stack with continuously stacked autocorrelations $(\mathrm{a}, \mathrm{b})$ and cross-correlations $(\mathrm{c}, \mathrm{d})$. Blue lines indicate the threshold, after which the median RMS-difference permanently decreases to below 0.1 .

overall characteristics of the waveforms are similar, especially for the East-West component. Around 12-13 s lag time, a clear decrease in amplitude with a subsequent increase can be detected. At lag times of 23-24 s, the amplitude of this wave package decreases again, to slightly increase again at around 27-28 s. Station GEC2 shows overall lower amplitudes, but the described features are also detectable. The mean correlation coefficient between the autocorrelations for these stations in the lag time 5-30 s is 0.9193 . This time range was chosen, because it excludes the sidelobes of the main peak in the first five seconds, but includes all the main features of the autocorrelations relevant for this work. For the North-South component autocorrelations, the different autocorrelations show slightly different properties. Again, at around 12-13 s a clear amplitude decrease is visible for all stations, with a subsequent increase. This amplitude increase does not occur at the same time or in the same way at all stations. Up to this increase the correlations of all stations look similar, but vary afterwards. Stations GEA2 and GED7 show a distinct increase around $15 \mathrm{~s}$, with the amplitude rapidly decreasing again after. Station GED1 shows a similar increase, but the subsequent decrease is more gradual. At station GEC2, the later decrease is only very small and resembles a beat. At station GED4, the amplitude increase is only minimal and the amplitude stays at a constant level afterwards. Around 23-24 s, stations GEA2 and GED7 show a decrease, similar to the East-West component correlations. Stations GEC2 and GED1 do not show any visible changes within this lag time. The mean correlation coefficient for North-South component autocorrelations between $5-30 \mathrm{~s}$ lag time is 0.8009 .

The cross-correlations of mixed components cannot be compared to each other for the GERESS stations. The number of correlations available is too small to obtain a stable result. Due to the high number of data gaps, only a small number of cross-correlations can 
(a)

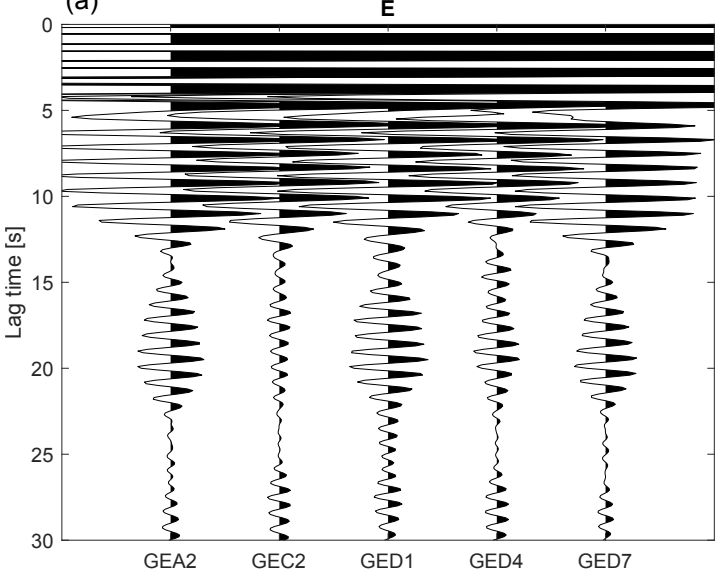

(b)

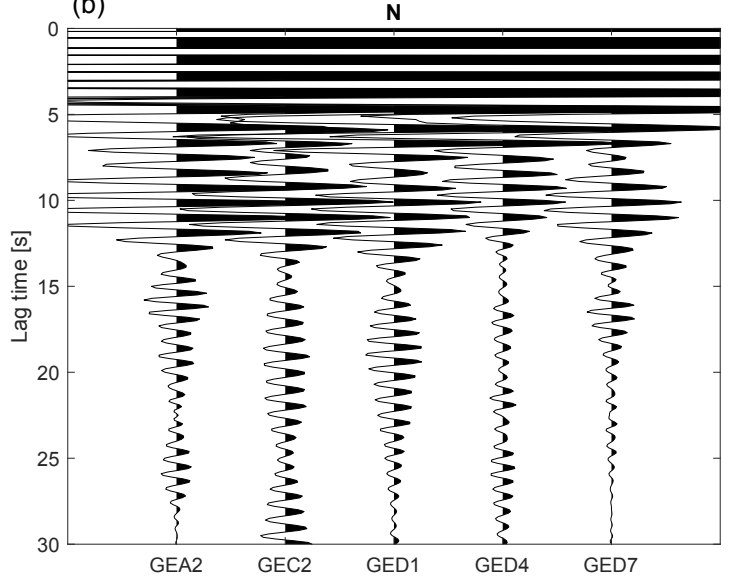

Figure 3.5: Wiggle plots of the calculated horizontal component autocorrelations for the three component stations of the GERESS array. (a) East-West component, (b) North-South component.

be calculated. Only for the broad-band station GEC2, the number of correlations is sufficient for a stable result. In general, the cross-correlations for the different stations look very different in shape and amplitude, which can be associated with the cross-correlations not being stable, yet. At station GED7 the number of cross-correlations of horizontal component data is close to the determined threshold (Figs 3.4c and d) and the result is starting to show overall similar characteristics as station GEC2. For the rest of the stations and cross-correlations, no definite statement can be made.

\subsubsection{Consistency across correlations}

Assuming that the seismic waves reaching the seismometers do not arrive from directly vertically below the station, it would be possible to detect $P$-wave reflectivity changes on horizontal component auto- and cross-correlations, $S$-wave reflectivity changes on vertical component autocorrelations, and reflectivity changes associated with $P$-to- $S$ or $S$-to- $P$ converted waves on mixed horizontal and vertical component cross-correlations. It is indeed possible to detect these phases for some stations in the $1-2 \mathrm{~Hz}$ frequency range. We detect these phases by over-laying the determined target lag times onto the correlations and picking reflectivity changes within $\pm 2 \mathrm{~s}$ to the centre of the result window. As a final step, only those reflectivity changes occurring within $\pm 0.5 \mathrm{~s}$ of the determined result windows are considered to be the same phase and therefore relevant. Reflectivity changes earlier or later than the result window by more than $0.5 \mathrm{~s}$ might be related to different phases and are not considered further.

\subsubsection{Vertical-horizontal component cross-correlations}

For the vertical-horizontal cross-correlations we calculate the expected lag times of reflectivity change by taking half of the $P$-reflectivity lag times determined in Becker $\&$ Knapmeyer-Endrun (2018) and half of the $S$-reflectivity lag times determined here and add them together. We then plot them onto the vertical-horizontal component crosscorrelations, to verify whether a reflectivity change is also detectable within the bounds 

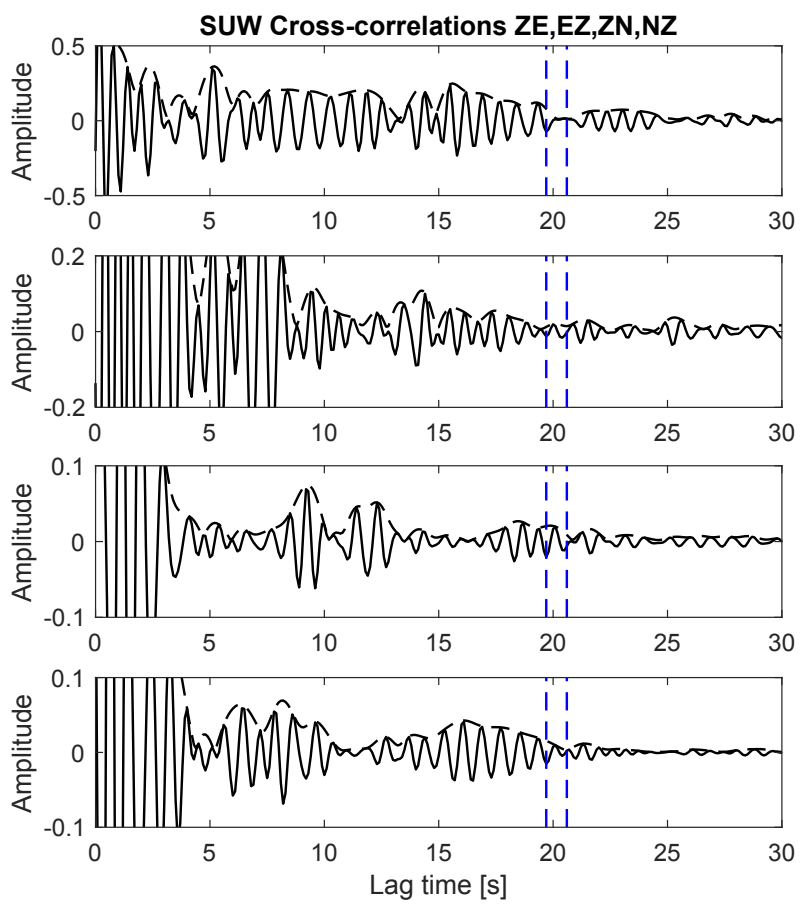

Figure 3.6: Vertical-horizontal components cross-correlations (black) of station SUW. Black dashed line: envelope. Blue lines: Calculated expected lag times from vertical component autocorrelation and horizontal component auto- and cross-correlation picks.

given by those lag times. An example for this can be seen in Fig. 3.6. Here, a reflectivity change is clearly visible within the determined result window (blue lines) on the Z-E and $\mathrm{N}-\mathrm{Z}$ correlation. On the E-Z and Z-N correlation, a reflectivity change occurs within $\pm 0.3 \mathrm{~s}$. In all correlations, a minimum in the cross-correlations characterizes the reflectivity change. Considering all stations, 14 out of the 100 correlations show a reflectivity change directly within the determined result window and stations BEL, SUW and VSU each show this reflectivity change on two correlations. Another 33 correlations show a reflectivity change within $\pm 0.5 \mathrm{~s}$ of the result window. Station BSEG does not show any reflectivity change within \pm 0.5 s of the result window on all four correlations and ten stations show a reflectivity change only on one correlation. Stations BEL, SUW and WET are the only stations where a reflectivity change close to the result window occurs on all four correlations. The remaining stations show reflectivity changes on two or three correlations. This leads to on average of 1.9 detections of a reflectivity change associated with a converted phase per station.

\subsubsection{Horizontal component auto- and cross-correlations}

In order to detect possible $P$-wave reflectivity changes on the horizontal component autoand cross-correlations, the lag times determined in Becker \& Knapmeyer-Endrun (2018) are over-plotted onto the horizontal component correlations and second derivatives. We then check whether a maximum in the second derivative is found at the determined lag time or within $\pm 2 \mathrm{~s}$ of the former, and whether this coincides with a clearly visible reflec- 

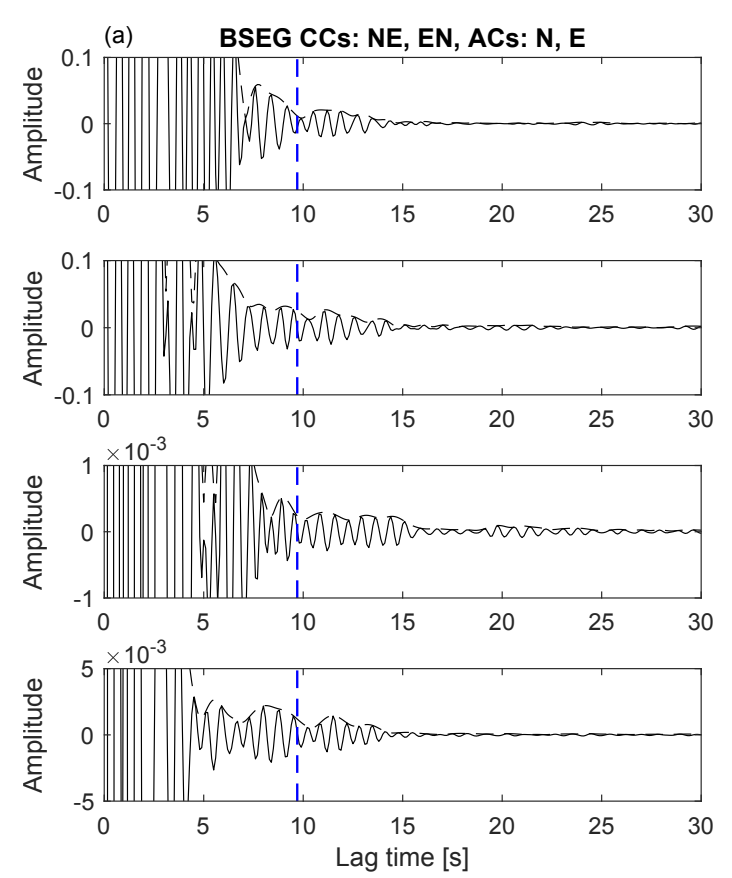
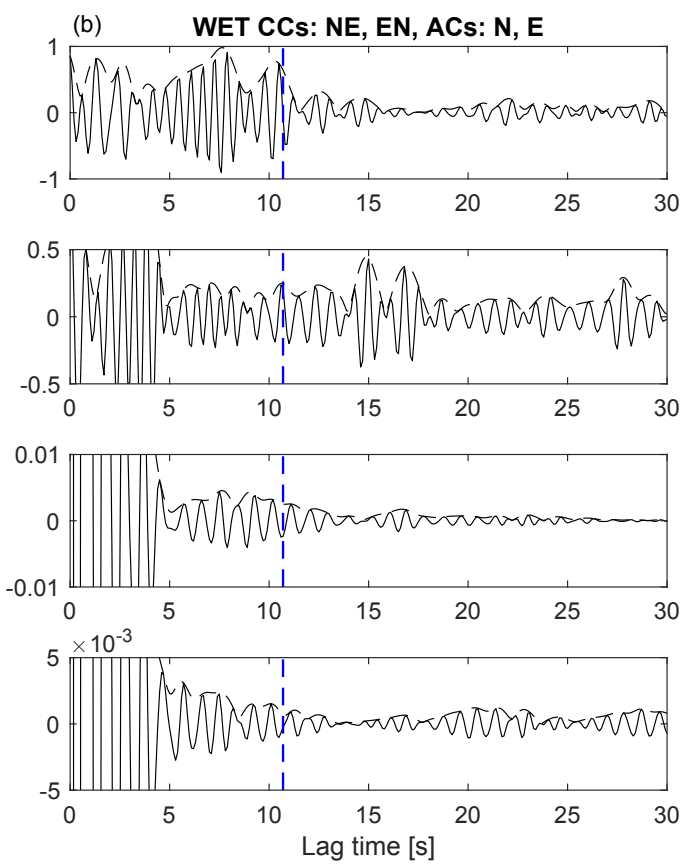

Figure 3.7: Horizontal component cross-correlations (top rows) and autocorrelations (bottom rows) for stations BSEG (left) and WET (right). Black dashed lines: envelopes. Blue lines: Expected lag time of $P$-wave reflectivity change (Becker \& Knapmeyer-Endrun 2018).

tivity change in the correlations. Not for all stations this reflectivity change is detectable and especially not on all components. This is illustrated for station WET in Fig. 3.7(b). Close to the determined expected lag time (blue line), only the NE-correlation shows a clear reflectivity change, but at a later lag time than expected. At station BSEG (Fig. 3.7a) a reflectivity change is detectable within $\pm 2 \mathrm{~s}$ of the expected lag time on all correlations, but only the NE-cross-correlation and the $\mathrm{N}$-autocorrelation show this reflectivity change within $\pm 0.5 \mathrm{~s}$ of the expected lag time. Of all horizontal component correlations, five correlations show a reflectivity change exactly at the expected lag time and on another 31 correlations a reflectivity change can be detected within $\pm 0.5 \mathrm{~s}$ of the expected lag time. Five stations (GKP, MORC, SUW, VSU, WET) do not show any reflectivity change within $\pm 0.5 \mathrm{~s}$ of the expected lag time. Stations CLL, KRUC, PABE, PBUR, SLIT, TNS and WLF show a reflectivity change within that range only on one correlation. For the remaining stations, a reflectivity change can be detected on 2-3 correlations. On average 1.4 correlations per station show a reflectivity change within $\pm 0.5 \mathrm{~s}$ of the expected lag time.

\subsubsection{Vertical component autocorrelations}

\subsection{4 $v_{\mathrm{P}} / v_{\mathrm{S}}-$ ratios}

A range of $v_{\mathrm{P}} / v_{\mathrm{S}}$-ratios was calculated for each station by taking the picked lag times of the horizontal component correlations and the picked lag times of the vertical autocorrelations from Becker \& Knapmeyer-Endrun (2018). An overview of obtained values can be found in Table 3.1. The theoretical $v_{\mathrm{P}} / v_{\mathrm{S}}$-ratios calculated from the $P$-wave velocities used in Becker \& Knapmeyer-Endrun (2018) and the $S$-wave velocities taken from 

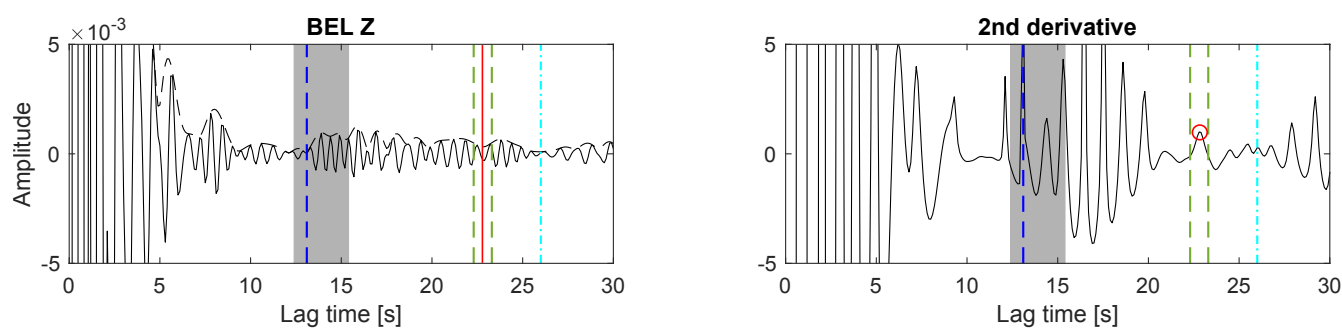

Figure 3.8: Vertical component autocorrelation with envelope (black, dashed) of station BEL (left) with corresponding second derivative. Red line/circle: Picked $S$-wave reflectivity change. Grey: $P$-wave reflectivity change target window; dark blue line: picked lag time of $P$-wave reflectivity change; green lines: picked lag times of $S$-wave reflectivity change; light blue line: picked LAB reflectivity change of $P$-wave

Table 3.1: Overview of Moho depths determined for the broad-band stations. Moho depths of $P$-reflections taken from Becker \& Knapmeyer-Endrun (2018). Moho depths from $S$-reflections calculated from the picked lag times in the auto- and cross-correlation results and velocity values taken from Molinari \& Morelli (2011). $v_{\mathrm{P}} / v_{\mathrm{S}}$-ratios calculated from picked lag times of $P$ - (Becker $\&$ Knapmeyer-Endrun 2018) and $S$-reflections (this study).

\begin{tabular}{lccccc}
\hline Station & $\begin{array}{c}\text { Moho depth }[\mathrm{km}] \\
\text { Vertical } \\
\text { component }\end{array}$ & $\begin{array}{c}\text { Moho depth }[\mathrm{km}] \\
\text { Horizontal } \\
\text { components }(\min )\end{array}$ & $\begin{array}{c}\text { Moho depth }[\mathrm{km}] \\
\text { Horizontal } \\
\text { components }(\max )\end{array}$ & $\begin{array}{c}v_{\mathrm{P}} / v_{\mathrm{S}} \text {-ratio } \\
(\mathrm{min})\end{array}$ & $\begin{array}{c}v_{\mathrm{P}} / v_{\mathrm{S}} \text {-ratio } \\
(\mathrm{max})\end{array}$ \\
\hline BEL & 40.7 & 40.4 & 42.2 & 1.70 & 1.78 \\
BFO & 27.5 & 23.2 & 24.7 & 1.45 & 1.55 \\
BRG & 31.9 & 29.7 & 31.3 & 1.59 & 1.70 \\
BSEG & 28.6 & 28.7 & 30.4 & 1.72 & 1.82 \\
BUG & 30.1 & 31.1 & 32.5 & 1.79 & 1.87 \\
CLL & 33.2 & 29.8 & 32.1 & 1.55 & 1.67 \\
CLZ & 32.4 & 30.3 & 32.7 & 1.61 & 1.74 \\
FBE & 29.6 & 26.6 & 28.7 & 1.55 & 1.67 \\
GEC2 & 38.7 & 36.7 & 37.4 & 1.64 & 1.67 \\
GKP & 36.0 & 36.0 & 37.6 & 1.74 & 1.81 \\
KHC & 39.3 & 38.3 & 38.5 & 1.81 & 1.82 \\
KRUC & 33.6 & 32.2 & 33.9 & 1.72 & 1.81 \\
MORC & 35.3 & 36.0 & 37.9 & 1.82 & 1.91 \\
MOX & 33.5 & 30.0 & 30.0 & 1.55 & 1.55 \\
PABE & 48.0 & 44.3 & 47.3 & 1.60 & 1.71 \\
PBUR & 49.5 & 44.5 & 49.6 & 1.57 & 1.75 \\
SLIT & 43.7 & 44.5 & 45.6 & 1.76 & 1.81 \\
STU & 24.8 & 28.7 & 29.4 & 2.00 & 2.05 \\
SULZ & 27.0 & 24.8 & 26.0 & 1.59 & 1.66 \\
SUW & 49.4 & 46.4 & 49.8 & 1.64 & 1.77 \\
TNS & 26.8 & 24.9 & 27.1 & 1.61 & 1.76 \\
UBBA & 32.8 & 31.3 & 33.3 & 1.66 & 1.76 \\
VSU & 43.4 & 50.4 & 53.0 & 2.01 & 2.11 \\
WET & 32.4 & 30.7 & 31.8 & 1.64 & 1.69 \\
WLF & 30.7 & 34.4 & 34.8 & 1.93 & 1.95 \\
\hline & & & & &
\end{tabular}


Molinari \& Morelli (2011) vary between 1.72 and 1.86 . The majority of stations show $v_{\mathrm{P}} / v_{\mathrm{S}}$-ratios of 1.72-1.74. The minimum value calculated from the correlations is 1.45 at station BFO and the maximum is 2.11 at station VSU. Another twelve stations show a $v_{\mathrm{P}} / v_{\mathrm{S}}$-ratio lower than 1.65 , and one other station gives a $v_{\mathrm{P}} / v_{\mathrm{S}}$-ratio larger than 2 . These values are not realistic. Other studies (Wilde-Piórko et al. 2005, Lombardi et al. 2008, Geissler et al. 2008, Knapmeyer-Endrun et al. 2014) obtained $v_{\mathrm{P}} / v_{\mathrm{S}}$-ratios between 1.65 and 1.94 from receiver functions for the same stations. In addition, the ranges of $v_{\mathrm{P}} / v_{\mathrm{S}^{-}}$ ratios obtained are larger than or equal to 0.1 for 11 out of 25 stations, which makes it hard to derive any constraints on the crustal composition.

\subsubsection{Moho depths}

In contrast to the autocorrelations from vertical component data, where a specific Moho depth is calculated, only a depth range can be provided here. The limits of these ranges can be seen as two different symbols for each station in Fig. 3.9 and are listed in Tab. 3.1. In the map, the circles indicate the deeper boundary, with the triangles representing the upper boundary. In general, the depths match well with the Moho depths provided by the European Moho depth map by Grad et al. (2009). The mean and standard deviations of the depth difference are $-1.2 \pm 2.0 \mathrm{~km}$ and $0.5 \pm 2.2 \mathrm{~km}$ with the maximum and minimum differences found at stations VSU with $5.8 \mathrm{~km}$ and PABE with $-4.6 \mathrm{~km}$. Fig. 3.10 compares the Moho depths obtained here, assuming vertical incidence, with depths determined in previous studies. The black symbols indicate the uncertainty originating from the picks on the horizontal component auto- and cross-correlations. The green symbols indicate the depth uncertainty resulting from the $5 \%$ velocity uncertainty used in the conversion from lag time to depth in case of the depths determined from correlations. In the case of the European Moho depth map, all stations fall very close to the line with slope 1, the line of best fit has a slope of 1.0104 and an offset of -0.7390 (Fig. 3.10b). The negative offset shows that the depths determined here are on average smaller than those predicted in the European Moho depth map. When comparing the determined depth to results obtained from receiver functions by Knapmeyer-Endrun et al. (2014) the mean and standard deviations of the depth differences are $0.6 \pm 3.0 \mathrm{~km}$ and $2.4 \pm 3.1 \mathrm{~km}$. The highest difference is found at station WLF with $10.8 \mathrm{~km}$ and the lowest deviation at station WET with $-3.3 \mathrm{~km}$. In comparison to the results obtained by Knapmeyer-Endrun et al. (2014) the depths here are overall larger. This can also be seen in Fig. 3.10 c) represented by the red line of best fit, being shifted up from the black line with slope 1. It has a slope of 0.9836 and an offset of 3.5677. In addition to Moho depths from other studies and methods, we also compare the results to those obtained with the same method from vertical component autocorrelations in Becker \& Knapmeyer-Endrun (2018). The mean depth differences are $-1.0 \pm 2.8 \mathrm{~km}$ and $0.7 \pm 2.6 \mathrm{~km}$. The minimum difference is $-5.0 \mathrm{~km}$ at station PBUR and the maximum difference is $9.6 \mathrm{~km}$ at station VSU. Fig. 3.10(a) shows the direct comparison of the two data sets, including velocity uncertainty. Most of the stations fall very close to the line of slope 1. The outlier in the upper half is station VSU, where the difference is rather large. The line of best fit has a slope of 0.9973 and an offset of -0.031 . The depths obtained here are on average slightly smaller than those obtained from vertical component autocorrelations.

For non-vertical incidence, the incidence angle influences the calculation of depth 


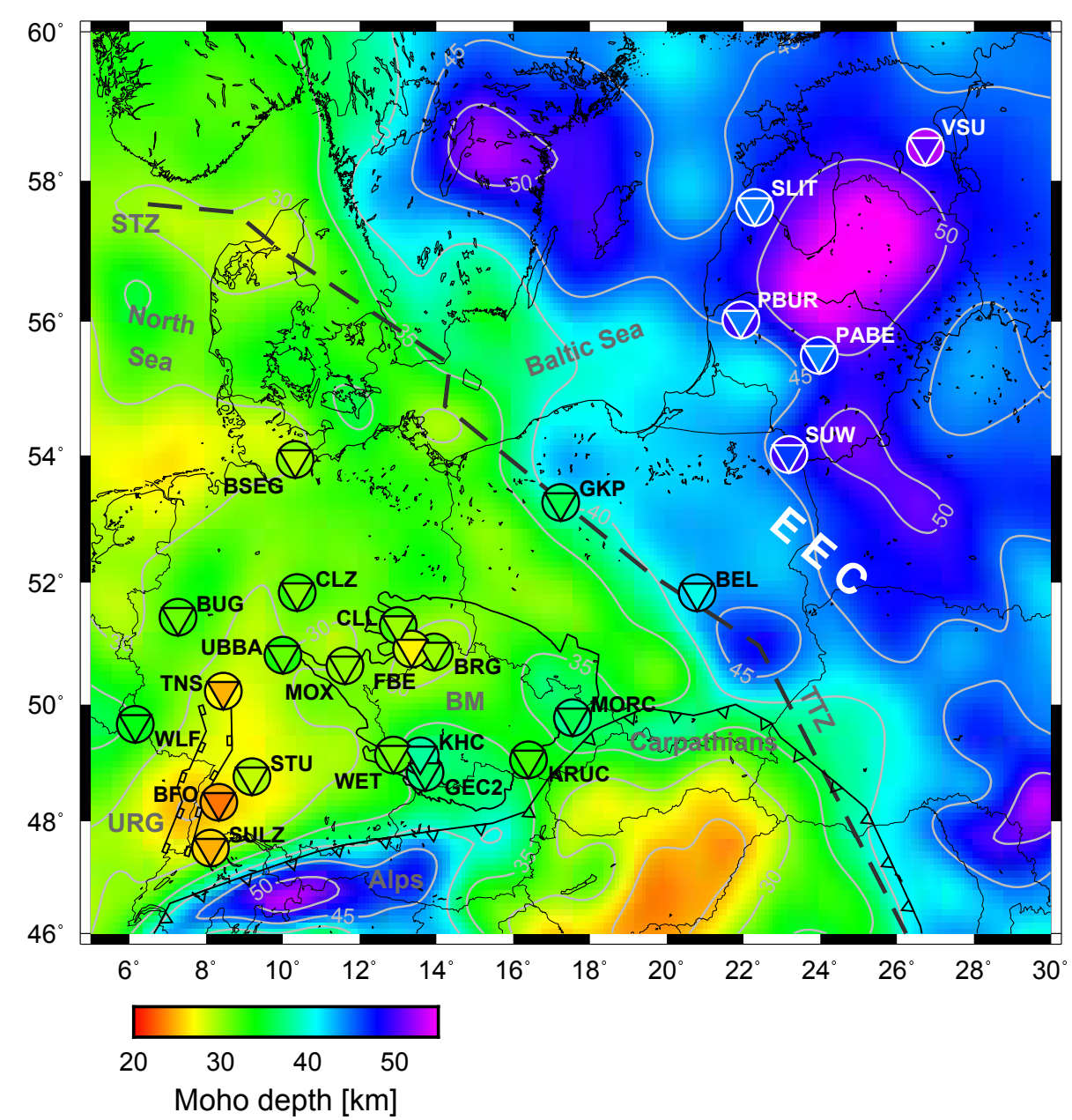

Figure 3.9: Moho depth ranges obtained from auto- and cross-correlations of horizontal components over-plotted on the European Moho depth map after Grad et al. (2009). Circles indicate lower boundary, triangles upper boundary. Labels: BM: Bohemian Massif; EEC: East European Craton; STZ: Sorgenfrei-Tornquist-Zone; TTZ: Teisseyre-Tornquist-Zone; URG: Upper Rhine Graben.

from lag time. If we consider incidence angles between $0^{\circ}-35^{\circ}$, the determined depths vary by up to $18 \%$.

\subsection{Discussion}

The processing applied here was originally developed for the calculation of autocorrelations of vertical component data, but overall also works well for the autocorrelations of horizontal component data and is easily adjustable for single-station cross-correlations. The main draw-back is that the automated picking process of reflectivity changes developed for the vertical component autocorrelations cannot be applied here. For the horizontal component correlations multiple traces are available, which need to be visually compared by the analyst to detect correlated reflectivity changes present on all traces. Those reflectivity changes do not occur at exactly the same time on all correlations and can therefore not be retrieved by stacking the different correlations. 

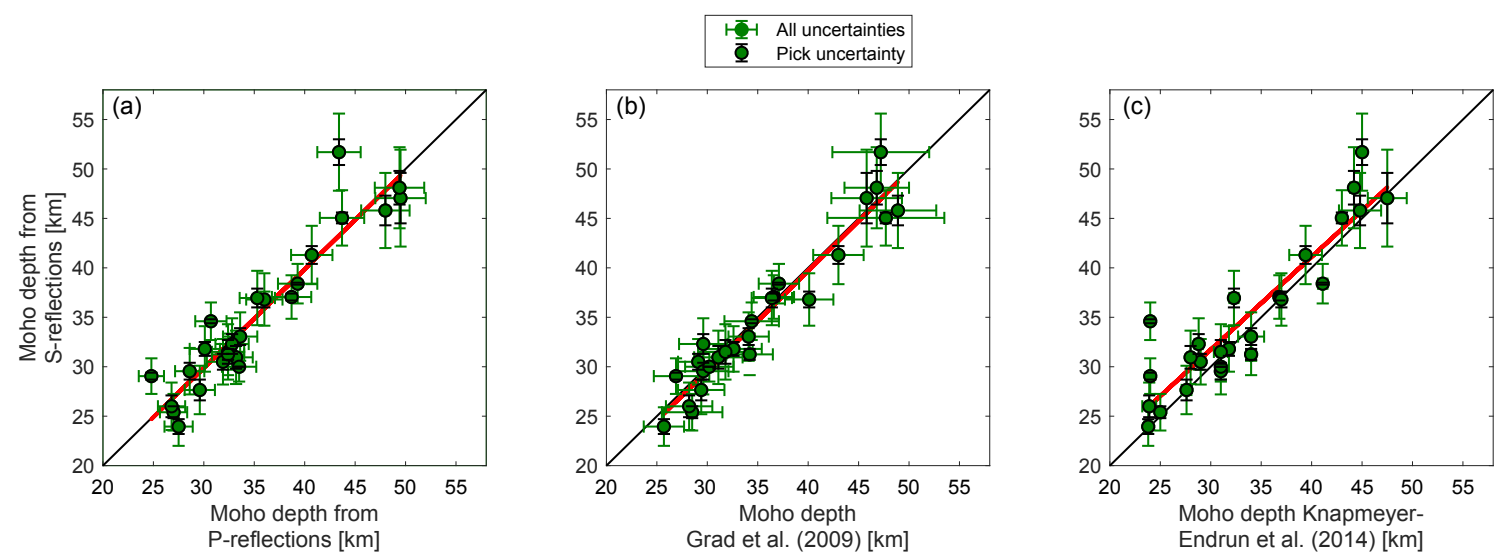

Figure 3.10: Comparison of Moho depths determined here with results from Becker \& Knapmeyer-Endrun (2018) (a), Grad et al. (2009) (b) and Knapmeyer-Endrun et al. (2014) (c). Black symbols indicate uncertainty originating from the two picks of the horizontal component correlations. Green symbols indicate uncertainty resulting from uncertainty in the velocity used for converting lag time to depth, as well as uncertainty provided by the studies. The black lines have a slope of 1 , whereas the red lines indicate the lines of best fit.

Filtering the correlations between 1-2 Hz gives the best result, as was also the case for the vertical component autocorrelations (Becker \& Knapmeyer-Endrun 2018). The frequency band of $2-4 \mathrm{~Hz}$ is used for picking the $S$-reflectivity changes on the horizontal component correlations when no coinciding reflectivity change across the four correlations for a single stations, or no reflectivity change at all, is detectable in the $1-2 \mathrm{~Hz}$ frequency band. For $\sim 80 \%$ of the traces, where no reflectivity change could be detected in the frequency band of 1-2 Hz, a detection can be made in this frequency band. This equals $\sim 1 / 3$ of all horizontal correlations. The higher frequency band can thus be used in addition to the lower frequency band to provide further estimates of reflectivity change lag times. Further considering a lower frequency band of $0.5-1 \mathrm{~Hz}$ does not provide any additional information. Only for one station a reflectivity change could be detected which is not detectable within the two higher frequency bands. Therefore this frequency band is not considered for the detection of reflectivity changes.

The temporal properties observed from the horizontal component correlations are overall similar to the ones observed from the vertical component autocorrelations in Becker \& Knapmeyer-Endrun (2018). Most stations show higher variances during the northern hemisphere summer. These higher variances and coinciding higher ambient noise can be explained by a higher cultural noise level in the summer due to, for example, construction sites and traffic being more active. Some of the stations showing higher variances in the northern hemisphere winter (SLIT, VSU, SUW) are stations located relatively close to the coast. The higher noise in the winter there can be explained by a larger number of waves hitting the shore and these waves also being higher. Some stations (BSEG, GKP, PBUR, TNS) show different temporal behaviour with respect to variance amplitude, when comparing the vertical component autocorrelations to the horizontal component autocorrelations. This indicates that the different components are influenced by different noise sources. The noise that is considered here in the frequency band of 1-2 Hz consists of cultural as well as natural noise (Bonnefoy-Claudet et al. 2006). This natural noise 
can contain, for example, an increased number of waves hitting the shore in the winter, pressure variations or stronger winds (Iyer \& Healy 1972), leading to the increase in variance. In general, we conclude that most of the stations are influenced by cultural noise, because the Lomb-Scargle-periodograms show frequency peaks at weekly and daily intervals, which can easily be explained by human activity being higher during the week and day. This also means that the noise wave-field used for the calculation of correlations is non-stationary with time.

The results of the analysis of the three-component stations of the GERESS-array, in combination with the number of correlations needed to obtain a stable result, have several implications. The number of horizontal component autocorrelations needed for a stable result with a median RMS-difference smaller than 0.1 is approximately $30 \%$ higher than for the vertical component autocorrelations. This already shows that the calculation of horizontal component autocorrelations is more complex than that of vertical component autocorrelations, and more data are needed. By comparing the autocorrelations of the different components of the GERESS-array, we see that the East-West-component autocorrelations have a higher mean correlation coefficient between 5-30 s lag time than the North-South component autocorrelations. The mean correlation coefficient of the vertical component autocorrelations of the same stations is even higher at 0.9434. Although the stations, processing and parameters used are exactly the same, the mean correlation coefficients vary significantly. By looking at the waveforms of the autocorrelations, it can be seen that the they show the same main characteristics, but vary in detail, especially the North-South component autocorrelations. It is difficult to say why the horizontal component autocorrelations of the five stations look different to each other, whereas the vertical component autocorrelations exhibit a very high correlation. The horizontal component autocorrelations converge to a stable result within the amount of data available, so the difference in waveform between the stations is not due to the stacks not having reached the stable point. The single horizontal component autocorrelations before stacking already show differences in waveform between the stations, which is not the case for the vertical component autocorrelations. The raw data used for the calculation of the autocorrelations do not give any additional information on the origin of the differences. On the one hand, the vertical component data show overall a slightly higher correlation coefficient for the short-period stations, than the horizontal component data. On the other hand, the raw data of stations GEA2 and GED7 for the East-West component show a very low correlation coefficient, but the two autocorrelations show the second highest correlation coefficient of the East-West component autocorrelations. No trend is observable with respect to similarity of the raw data compared to similarity of the autocorrelations. This illustrates again, that the horizontal component autocorrelations are more complex and more difficult to interpret.

The cross-correlations require even more data than the horizontal component autocorrelations. Especially the cross-correlations of the horizontal components need almost twice as much data as the horizontal component autocorrelations to reach stability. The cross-correlations of vertical with horizontal component data require approximately one third more data than the horizontal component autocorrelations and one fifth less than horizontal component cross-correlations. This might be attributed with the fact that the vertical component autocorrelations converge faster and that this behaviour also influences the cross-correlations, thereby making them converge faster than the purely horizontal 
component cross-correlations.

Ideally, the autocorrelations would only show the reflectivity changes of the waves with the corresponding polarization. The vertical component autocorrelations would show the $P$-wave reflectivity change and the horizontal component autocorrelations the $S$-wave reflectivity changes. The fact that we can clearly identify $S$-wave reflectivity changes on approximately half of the vertical-component autocorrelations and $P$-wave reflectivity changes on the majority of horizontal component auto- and cross-correlations, leads to the conclusion that these waves do not reach the stations from directly vertically below, but arrive at a variety of incidence angles. This is also supported by the observation of converted phases, $S$ - to $P$ - and vice versa, on the cross-correlations of horizontal with vertical components. These phases would not exist if the waves arrived from directly vertically below the stations. Tibuleac \& von Seggern (2012) also observed that two arrivals, which they interpret as Moho-reflected $P$ - and $S$-waves $(P \mathrm{~m} P, S \mathrm{~m} S)$, are visible on the horizontal as well as vertical component. In their polarization analysis the main incidence angles are $50^{\circ}-60^{\circ}$ for the $P \mathrm{~m} P$ arrivals and $30^{\circ}-50^{\circ}$ for the $S \mathrm{~m} S$ arrivals, confirming non-vertical incidence.

If the waves do not arrive from directly vertically below the station, this results in errors when converting lag times to depths, because during the conversion from lag time to depth vertical incidence is assumed. The reflectivity change occurs at later lag times, which then results into overestimated depths. Oren \& Nowack (2017) determined that the region of stationary phase includes contributions from a wide range of distances from the station, of up to $\sim 100 \mathrm{~km}$, depending on the frequency. We consider station BFO as an example, where the results for the depth, determined from the $S$-wave and $P$-wave picks, differ by $\sim 3 \mathrm{~km}$ (Tab. 3.1), assuming vertical incidence, and $v_{\mathrm{P}} / v_{\mathrm{S}}$-ratios are very low at 1.45 to 1.55 . If we now assume that the $P$-waves reach the surface with an incidence angle of $30^{\circ}$, the resulting Moho depth would be $23.8 \mathrm{~km}$, which would fall right in between the two depths estimates obtained with vertical incidence from the $S$-wave. The new $v_{\mathrm{P}} / v_{\mathrm{S}^{-}}$ ratio would be 1.67, which is very close to the estimates by Lombardi et al. (2008) and Geissler et al. (2008). In this example, we assume that one of the wave types arrives at the surface from vertically below, which would result in the correct depth using the simple linear relation and assuming vertical incidence. However, both wave types could reach the surface at an angle, resulting in overestimated depths. This shows that assumptions on the incidence angle play an important role in the calculation of depth and the $v_{\mathrm{P}} / v_{\mathrm{S}}$-ratio.

Nevertheless, when comparing the Moho depths here with the depths provided in the European Moho depth map (Grad et al. 2009), a slight negative offset of the line of best fit can be observed. Slightly more than half of the stations (14 out of the 25) show a negative difference between the depth determined here and the depth provided in the map. A similar trend is observed, when comparing the results to those obtained in Becker \& Knapmeyer-Endrun (2018). A small negative offset of the line of best fit is detectable, with 16 out of 25 stations showing a negative difference of mean determined depth. Only the comparison with the results by Knapmeyer-Endrun et al. (2014) shows a shift to greater depths and a positive offset of the line of best fit, which can also be observed in Fig. 3.10. Here, 15 out of the 25 stations show a positive difference of mean determined depth. One of the factors that could lead to differences in depths in general is the $S$-wave velocity used for the conversion of lag time to depth. We use $S$-wave velocities provided in the EPcrust model (Molinari \& Morelli 2011). The resolution of the grid used 
there is $0.5^{\circ} \times 0.5^{\circ}$ and the $S$-wave velocities are calculated using a scaling relation with respect to the $P$-wave velocities. This may lead to lower variability in $S$-wave velocity, due to the nature of the scaling relation, compared to $P$-wave velocities determined from other methods such as, for example, refraction seismics. Nevertheless, it seems that overall velocity uncertainties and possible non-vertical incidence do not result in large deviations from expected Moho depths.

The non-vertical incidence plays a stronger role in the calculation of $v_{\mathrm{P}} / v_{\mathrm{S}}$-ratios. A comparison with obtained ratios from other studies shows large deviations in $v_{\mathrm{P}} / v_{\mathrm{S}}$-ratios for some stations. In general, a large variability between the results obtained here compared to values obtained for the same stations by Wilde-Piórko et al. (2005), Lombardi et al. (2008), Geissler et al. (2008) and Knapmeyer-Endrun et al. (2014) can be observed, with a majority showing slightly lower values. The obtained $v_{\mathrm{P}} / v_{\mathrm{S}}$-ratios are on average 0.02 smaller than those obtained for the same stations by the studies mentioned above. Out of the $51 v_{\mathrm{P}} / v_{\mathrm{S}}$-ratios determined by those studies, 9 ratios fall directly within the $v_{\mathrm{P}} / v_{\mathrm{S}}$-ratio ranges obtained here and 24 ratios are within 0.1 of the ranges. Stations VSU and STU show much higher $v_{\mathrm{P}} / v_{\mathrm{S}}$-ratios than determined by the other studies. A deviation in ratio of up to $20 \%$ can be observed. Other stations such as, for example, BFO and MOX show $v_{\mathrm{P}} / v_{\mathrm{S}}$-ratios that are much smaller than predicted by other studies. In addition, the ranges of the $v_{\mathrm{P}} / v_{\mathrm{S}}$-ratios are large, with a range of up to $11.5 \%$ for station PBUR. It becomes apparent that the results of the $v_{\mathrm{P}} / v_{\mathrm{S}}$-ratio calculation need to be treated carefully, as large deviations and ranges and unrealistic values are present. Also neighbouring stations on the same geological unit, such as, for example, BFO and STU, show very different values, which is another indication for the questionability of these results. A similar observation was made by Tibuleac \& von Seggern (2012). The $v_{\mathrm{P}} / v_{\mathrm{S}}$-ratios calculated by Tibuleac \& von Seggern (2012) also show good agreement with results obtained by other studies for some stations, whereas for others the results vary. 36 out of their 66 stations show a deviation in $v_{\mathrm{P}} / v_{\mathrm{S}}$-ratio to the reference study of larger than or equal to 0.1 . This highlights again the difficulty of obtaining reliable $v_{\mathrm{P}} / v_{\mathrm{S}}$-ratios from autocorrelations.

When assuming that the incidence of the waves is vertical, the $P$ - and $S$-waves would follow the same travel path and the calculation of the $v_{\mathrm{P}} / v_{\mathrm{S}}$-ratios would be simple. If the incidence is not vertical, but the two waves follow the same path with the same incidence angle, the result would be the same. If on the other hand we assume that $P$-and $S$-waves have different incidence angles by, for example, originating from different sources, the travel paths of the two waves would be different and therefore result in an incoherent $v_{\mathrm{P}} / v_{\mathrm{S}}$-ratio. Assuming a specific stationary noise source, the incidence angle would be constant. However, as sources are non-stationary with time, the incidence angle might vary over time and and also be different for the two horizontal components. Whereas in the calculations of Moho depths, these differences in incidence angle do not influence the results much, for the calculation of $v_{\mathrm{P}} / v_{\mathrm{S}}$-ratios even a small difference results in large deviations. An example for this is station CLZ, where the results for the depths determined from the vertical and horizontal component correlations are very similar to each other and the depth range of $2.4 \mathrm{~km}$ is small, but the determined $v_{\mathrm{P}} / v_{\mathrm{S}}$-ratios, show a large range of 0.13 and deviate by more than 0.1 from the results in other studies. At some stations, for example stations PABE and VSU, it is not possible to observe converted phases on vertical-horizontal component cross-correlations and $\mathrm{S}$ - and $\mathrm{P}$-waves on vertical and horizontal component correlations, respectively. For these stations, vertical incidence could 
be assumed. Unfortunately, also these stations show deviations in $v_{\mathrm{P}} / v_{\mathrm{S}}$-ratios to values from other studies. Overall it can be said, that it is difficult to determine realistic $v_{\mathrm{P}} / v_{\mathrm{S}^{-}}$ ratios, due to the uncertainty in travel paths of the waves. The results for the Moho depths agree well with other studies, but it is not possible to draw conclusions about $v_{\mathrm{P}} / v_{\mathrm{S}}$-ratios and accordingly the mineral composition of the crust.

If instead of noise, seismic events with known epicentral distances are used for the calculation of auto- and cross-correlations, information about the incidence angle is available. This information can be used to reconstruct the vertical incidence reflection response, as is done by, for example, Sun \& Kennett (2016), and more reliable $v_{\mathrm{P}} / v_{\mathrm{S}}$-ratios could be obtained.

As discussed in section 3.5.3, it is possible to observe reflectivity changes of the different waves on correlations of multiple components, indicating non-vertical incidence. This leads to difficulties when looking for reflectivity changes associated with deeper layers, such as, for example, the LAB, because reflectivity changes associated with $S$ waves interfere with $P$-wave reflectivity changes associated with deeper layers, as is here the case for eight stations. This occurs mostly for stations with a shallow LAB depths, or when only one major reflectivity change is observable at later lag times. From this we can deduce that one has to be cautious when trying to identify deeper discontinuities, because it is not obvious which reflectivity changes are seen. Other studies treated this difficulty differently. Kennett (2015) extracted lithosphere-asthenosphere transition reflectivity properties from vertical component autocorrelations, but also concluded that interpretation of structures at asthenospheric depth is problematic and that contamination from shallower structure might influence the results. Tibuleac \& von Seggern (2012), who detected Moho-reflected $S$-waves on vertical component autocorrelations, dismiss the possibility of a reflection of a deeper layer, by the fact that previous studies in the area did not find evidence of such a layer.

When comparing the different lag times of reflectivity changes on the different autoand cross-correlations is becomes apparent that reflectivity changes being associated with the same layer do not always occur at exactly the same time. This could be a sign of non-vertical or varying incidence of the wave types, as explained above, and be related to non-stationary noise sources. In addition, it can be an indication for possible local anisotropy. Crustal anisotropy can have three different origins (Rabbel \& Mooney 1996): Firstly, sedimentary sequences consisting of layered rocks with varying stiffness can as a whole be anisotropic, even if the different layers are isotropic. Secondly, the occurrence of elastically weak zones in the rock due to tectonic stress, such as microcracks, fractures or inclusions, causes anisotropy. Lastly, the alignment of rock-forming minerals (textural ordering), for example as a result of of ductile deformation, is expected to be anisotropic.

In order to draw conclusions about possible azimuthal anisotropy below the stations, we compare the reflectivity change picks made on the two horizontal component autocorrelations. At all stations, except for SUW, reflectivity changes can be identified on the two components. If the two reflectivity changes occur at different lag times, those picks are converted to difference in velocity. An overview of direction and value of anisotropy overlayed onto the smoothed world stress map (Heidbach et al. 2010) can be found in Fig. 3.11. It is important to note that in this study, we can only distinguish between NorthSouth and East-West directions of anisotropy due to orientation of the components. The anisotropy is projected onto these two axes and a fast propagation direction of exactly $45^{\circ}$ 
beneath the station would not be detected. All the values obtained here are therefore minimum estimates of the amplitude of anisotropy. The obtained anisotropy varies between $0 \%$ at stations KHC, MOX and WLF to up to $6.67 \%$ at station BFO and $6.90 \%$ at station TNS as minimum estimates. The latter estimates are very high, especially because they are minimum estimates, and not realistic to only originate from anisotropy in the crust. Therefore, also other reasons might contribute to the differences in arrival time. Stations located in the Rhine Graben area show the same directionality. The reflectivity change occurs first on the East-West component autocorrelation. Only at station SULZ, the reflectivity change occurs earlier on the North-South component autocorrelation, although only by $0.1 \mathrm{~s}$. Stations BRG, CLL and FBE, which are located closely together in the Bohemian Massif, along with stations UBBA, WET and KRUC show the reflectivity change first on the North-South component autocorrelations. Stations MOX and KHC do not show any difference between lag times on the two components. Station GEC2 shows the reflectivity change first on the East-West component autocorrelation, but again the time difference is only $0.1 \mathrm{~s}$. Only station MORC shows a much earlier arrival on the EastWest component and a perpendicular anisotropy direction. The two stations located in the Polish Basin (GKP and BEL) also show reflectivity changes first on the North-South component autocorrelation.

When a material is exposed to an external stress field, fractures and cracks in the material will open in the direction of the minimum principal stress (Rabbel \& Mooney 1996). This leads to velocity anisotropy with a fast propagation direction parallel to the maximum compressional stress. The mean orientation of the maximum horizontal compressional stress is shown in the smoothed stress map of the study area in Fig. 3.11 (Heidbach et al. 2010). The comparison with the stress directions shows that the determined anisotropy aligns with the predominant direction of crustal stress for many stations. Stations GKP, BEL and BSEG, located in the basins, show anisotropy in N-S direction, which coincides with the direction on stress visible in the map. On the East European Craton the coverage of the stress map is sparse, compared to central Europe. Station VSU is not covered and station SLIT is at the edge of the stress map. Stations PBUR and SLIT show anisotropy in N-S direction, which is subparallel to the direction of stress. Station PABE exhibits anisotropy in E-W direction, which is roughly perpendicular to the direction of stress. This can be explained by the fact that in this area, the search radius used to calculate the smoothed stress map is rather large with a radius of $400-500 \mathrm{~km}$. The direction of stress indicated here is therefore an interpolation of measurements far away. Stations on the central European platform (BUG, CLZ, UBBA, WLF) show good agreement with the stress map. Station CLZ shows anisotropy in the E-W direction, which aligns very well with the measurements made in that area. Stations UBBA and BUG show N-S oriented anisotropy. This is comparable to the main direction of stress, which is oriented slightly towards NW. In the Bohemian Massif, the coverage of the stress map is also rather low. Stations CLL, FBE and BRG all show clear N-S oriented anisotropy. There are no stress estimates in close vicinity to compare this to. In the East of the Bohemian Massif the direction of stress rotates and shows mixed azimuths. Stations MORC and KRUC show perpendicular directions of anisotropy, which can be explained by the variable stress field. The anisotropy direction of station WET aligns very well with the surrounding stress field, whereas station GEC2 might also be affected by the rotating stress field. In the Rhine Graben, all stations except for station SULZ show anisotropy directions roughly perpendicular to the 


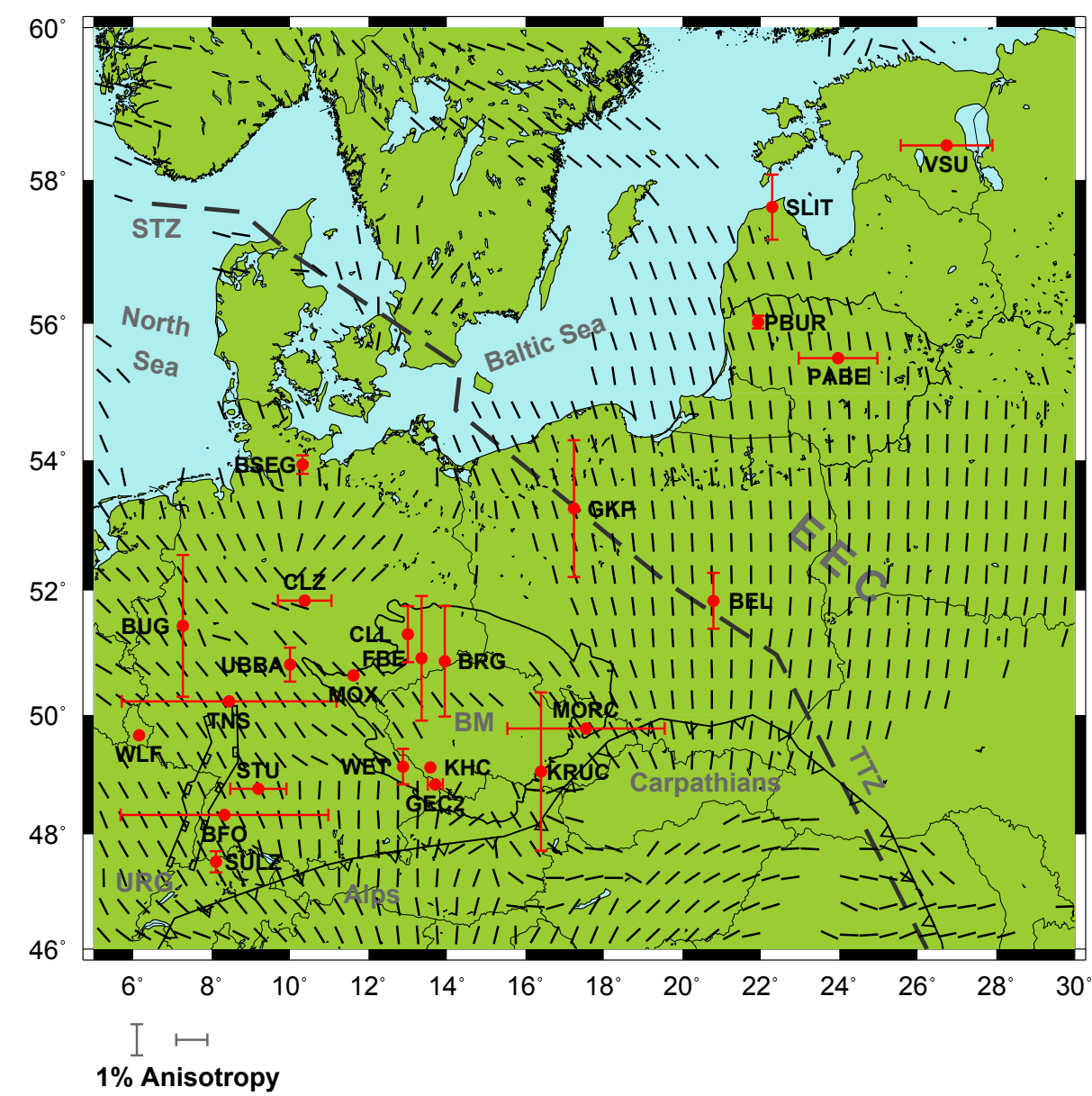

Figure 3.11: Overview of determined anisotropy orientation and value over-plotted onto the smoothed world stress map by Heidbach et al. (2010). The red lines are oriented in direction of the component registering earlier arrival, with the lengths proportional to percentage of anisotropy. For labels refer to Fig. 3.1.

stress field. A possible explanation could be a tilted Moho or a Moho with topography, associated with the rifting of the Graben, leading to lag times differences unrelated to anisotropy.

In this study we obtain anisotropy for the entire crust, whereas the stress map mostly considers the upper crust. Discrepancies in the direction of anisotropy could therefore also be explained by differences in anisotropy in the upper and lower crust, or by anisotropy not related to cracks and fractures. This was observed by Cristiano et al. (2016), who analysed $P$-wave polarization at the Gräfenberg Array in southern Germany and detected that the anisotropy in the brittle upper crust is more variable compared to the lower crust and mantle.

Růžek et al. (2000) determined the anisotropy for the Bohemian Massif from refraction travel time data, using travel times of $P g$ waves, and concluded that their observed anisotropy is related to the orientation of minerals and intrusions and not induced by the stress field. Their results shown an anisotropy of 1.5-2.5\% with a direction of maximum velocity propagation in $\mathrm{N} 35^{\circ} \mathrm{E}$. The here determined average anisotropy of $2.3 \%$ for the Bohemian Massif falls within that range, but is only a minimum estimate. The majority 
of stations show the reflectivity change on the North-South component first, which also agrees well with results obtained by Růžek et al. (2000). Station MORC is the only station showing a significant deviation from the N-S direction. It is located on the edge of the Bohemian Massif and may therefore be subject to a local variation in anisotropy. In addition, the results obtained by Rǔžek et al. (2000) are an average over the Bohemian Massif as a whole and may vary locally. Overall, the anisotropy determined here matches well with the smoothed world stress map (Heidbach et al. 2010) and the result determined by Růžek et al. (2000). The extraction of anisotropy from horizontal component autocorrelations provides an additional possibility to learn more about the subsurface structure of the crust below the station.

\subsection{Conclusions}

In this study we show that Moho-reflected $S$-waves can be extracted from single station horizontal component auto- and cross-correlations by identifying reflectivity changes consistent across all correlations within an uncertainty target window defined from a priori information. The obtained lag times of reflectivity changes are converted to depth and the results compare well with results obtained in other studies. In addition to the Moho-reflected $S$-waves on the horizontal component correlations, it is possible to detected reflectivity changes associated with converted body wave phases on horizontalvertical component cross-correlations, as well as $S$-wave reflectivity changes on vertical component autocorrelations and $P$-wave reflectivity changes on horizontal component correlations. This indicates non-vertical incidence of the waves, resulting in errors when converting lag times to depth, without additional information on the incidence angle.

The obtained autocorrelations are temporarily stable, but require more data than vertical component autocorrelations to reach stability. In addition, we calculate $v_{\mathrm{P}} / v_{\mathrm{S}}$-ratios from the determined lag times from the horizontal and vertical component correlations. However, these determined $v_{\mathrm{P}} / v_{\mathrm{S}}$-ratios show a large variability and partly give unreasonable values, so they have to be regarded with care. The large variability can be associated with the fact that $P$ - and $S$-waves do not travel along the same path, resulting in errors, when calculating $v_{\mathrm{P}} / v_{\mathrm{S}}$-ratios from lag times. The horizontal component autocorrelations could be used to detect crustal anisotropy, though, and are helpful when trying to interpret later arrivals on the vertical component autocorrelations in terms of deeper layers, by providing a means to validate that the correct $P$-phases, and not later arriving crustal $S$-phases, are being used. 



\section{Discussion and Outlook}

There is no law except the law that there is no law.

John Archibald Wheeler

This chapter will briefly summarise the results of chapters 2 and 3, before relating the results to each other and discussing them with respect to the InSight mission. In addition, an outlook regarding future work and possible extensions of the method will be given.

\section{Summary of results}

Vertical and horizontal component seismic noise data can be used to extract the crustal thickness underneath a single station. By incorporating prior information on suspected Moho depth and seismic velocities, reflectivity changes associated with the transition from crust to mantle can be detected in the auto- and cross-correlations of the seismic noise data. For the vertical component autocorrelations, the detection of those reflectivity changes can be done semi-automatically, whereas the horizontal correlations require the involvement of the analyst. In the case of vertical component autocorrelations, a single lag time for a reflectivity change is obtained for each station, which is converted to a Moho depth. The horizontal component correlations result in a range of lag times and depths, because all correlation results for the different horizontal components and their combinations need to be included.

The Moho depths obtained for the vertical and horizontal components in this work agree well with results determined by other studies. In addition to the crust-mantle interface, it is also possible to detect additional reflectors such as an intracrustal layer or the LAB from vertical component autocorrelations.

The vertical component autocorrelations are spatially stable and only show small differences between closely located stations. In addition, they are also similar for data from broad-band and short-period stations. The horizontal component autocorrelation results are spatially more variable than the vertical component results.

Generally, the majority of stations (vertical and horizontal component data) shows higher amplitudes in variance during the northern hemisphere summer, compared to the northern hemisphere winter. Changes in variance can be related to human activity being present during the day and week, leading to variance peaks at exactly the corresponding 
frequencies. Vertical component autocorrelations require on average $\sim 6$ months of data to reach a stable result. Horizontal component autocorrelations require $\sim 8.5$ months to reach a stable point, and the horizontal component cross-correlations require almost $\sim 17$ months of data.

In addition to the expected wave type on the relevant correlation, it is possible to observe reflectivity changes associated with converted phases on mixed horizontal-vertical component cross-correlations. It is also possible to detect reflectivity changes associated with $P$-waves on horizontal component correlations and with $S$-waves on vertical component auto-correlations for some stations. These observations are an indication for non-vertical incidence of the seismic waves on the free surface.

The two horizontal component autocorrelations for each station offer the possibility to detect crustal anisotropy projected onto the N-S or E-W directions. The determination of $v_{\mathrm{P}} / v_{\mathrm{S}}$-ratios from the chosen reflectivity changes on vertical and horizontal component correlations, does not give reliable results and requires caution.

\section{Discussion}

Sections $2.5 \& 3.6$ discuss the extraction of the Earth's crustal thickness from vertical and horizontal component correlations, respectively. Overall, both methods work well and lead to similar results. The horizontal component correlations are more complex and variable and require more data than the vertical component correlations. However, they provide additional means of identifying non-vertical incidence of seismic waves. Furthermore, horizontal component correlations can aid in distinguishing reflectivity changes associated with deeper layers from those related to $S$-waves and the Moho. In the following, the results obtained and discussed in chapters $2 \& 3$ will be reviewed with regard to the InSight mission and Mars.

Throughout this work, crustal thickness estimates were made for stations located in different geologic settings, with varying Moho depths between 23-53 km. This range covers a large part of the expected crustal thickness range for the InSight landing site. It shows that the extraction of the Moho depth from correlations is possible for stations located in different geologic settings with different crustal thicknesses. The similarity of the results obtained with the collocated broad-band and short-period seismometer shows that both data sets can be used, and possibly combined, to calculate the correlations.

A combined vertical channel of VBB/SP vertical component data will be continuously transmitted at $10 \mathrm{~Hz}$ (Knapmeyer-Endrun et al. 2017a). Vertical component data of this rate was used throughout this work and is sufficient to extract information about the crustal thickness, provided prior information in the form of velocity and predicted depth are available, as shown in chapter 2. The availability of such prior information is limited for Mars. Crustal thickness estimates for the landing site are available, for example, from gravity measurements (see chapter 1), but with uncertainty of a factor of 2-3. An initial velocity model is expected to be complete with three months of available data (Panning et al. 2017a). This model will be based on a limited dataset and contain large uncertainties. By including the reflection response obtained from the vertical component autocorrelations, the initial model could be refined and an estimate of crustal thickness could be made. However, with only the vertical component noise data and associated 
autocorrelations, it is difficult to match reflectivity changes at later lag times with the appropriate layer. It was shown in chapter 3, that those later changes can be related to deeper layers, or Moho-reflected $S$-waves. One possible way to distinguish between the layers is by considering the resulting $v_{\mathrm{P}} / v_{\mathrm{S}}$-ratio. If this ratio gives a very high or low, therefore unreasonable, value, the reflectivity change is probably not associated with Moho-reflected $S$-waves. In the case of InSight, three-component data of the VBB will be continuously transmitted at $2 \mathrm{~Hz}$ (Knapmeyer-Endrun et al. 2017a). The horizontal component data of this lower frequency data can possibly also be used to help in the allocation of reflectivity changes. In chapter 3 a lower frequency band was not considered further in the analysis, but for a few stations reflectivity changes could also be detected after filtering between $0.5-1 \mathrm{~Hz}$.

On Earth, the noise sources in the frequency band of 1-2 Hz can be of mixed cultural and natural origin (Bonnefoy-Claudet et al. 2006). It was shown in sections 2.4.2 \& 3.5.1 that the main noise source considered here is of anthropogenic origin. Changes in variance can clearly be related to intervals, such as, for example, weekdays and weekends, which can be explained with human activity. The noise sources on Mars will be of different origin (see section 1.3). On Earth, varying frequency bands have been used in different studies to extract body waves. From low, $(0.2-0.4 \mathrm{~Hz}$ in Taylor et al. (2016)) to higher frequency bands, (2-4 Hz in e.g. Gorbatov et al. (2013)), the frequency bands considered were chosen to suit the data and noise available. This is an indication that the frequency band might have to be adjusted for the Martian data, in order to accommodate the different noise environment. In addition, it was beneficial throughout this work and also applied in other studies, for example Sun \& Kennett (2017), to consider multiple frequency bands to obtain the best results. This approach can also be useful for other data sets, especially when the characteristics of the noise are unknown.

In addition to the question of a suitable frequency band, the amount of data needed to obtain a stable result is unclear. In this study, six months of vertical component data and approximately nine months of horizontal component data are needed to obtain stable autocorrelation results (see subsections 2.4.2 \& 3.5.1). Whether these numbers hold for Mars is debatable. On the one hand, seismic noise on Mars is expected to be lower than on Earth, providing less signal, as explained in section 1.3. This would result in a larger amount of data and consequently a longer time span needed to reach stability. On the other hand, the attenuation of the waves will be slightly lower than on Earth. The quality factor (Q) of Mars will be higher than that of Earth, but at most twice as high (Lognonné \& Mosser 1993). This indicates that seismic waves will be attenuated less and be able to reach the station from greater distances. As a consequence, this would decrease the amount of data needed to reach stability.

Stations located in basins or in areas with low-velocity sediment loads show a characteristic waveform shape in the vertical component autocorrelations (chapter 2). This can be associated with reverberations in the low-velocity sedimentary layer, influencing the waveforms. The shallow subsurface at the InSight landing site is thought to consist of fragmented regolith of at least 2-4 m and at most 12-18 m thickness (Warner et al. 2017), coarse ejecta, and fractured and unfractured basalt up to $200 \mathrm{~m}$ depth (Golombek et al. 2017, Knapmeyer-Endrun et al. 2017a). The velocities of the regolith, coarse ejecta and fractured basalt are thought to be much lower than those of the underlying bedrock (Delage et al. 2017, Kedar et al. 2017, Knapmeyer-Endrun et al. 2017a). This could lead to 
additional scattering and reverberations interacting within the shallow subsurface, thereby influencing the shape of the correlation waveforms. It is however questionable, whether a low-velocity layer of such a thickness will influence the waveforms in the frequency band used throughout this work, as the resulting wavelengths would be larger than the depth of that layer. The basins considered in this work contain sedimentary layers of multiple kilometres thickness. An additional sedimentary layer below 200 m (Knapmeyer-Endrun et al. 2017a), or in between volcanic material could however further enhance these reverberations and cause multiple reflections within the layer interacting with Moho reflections and thus altering the shape of the autocorrelations.

In addition to extracting reflected body waves from seismic noise correlations, they can also be used to determine temporal variability of the seismic velocities due to temperature changes. This has successfully been done for the Moon using auto- and crosscorrelations of the Apollo 17 data (Sens-Schönfelder \& Larose 2010). It was also shown for Earth in several studies (see section 1.5), that seismic noise autocorrelations can be used to detect temporal variations in seismic velocity. Another possible application of autocorrelations is in the detection of Mars' hum. Deen et al. (2017) observed the Earth's hum in autocorrelations of data from ocean bottom seismometers. Mars' hum level is expected to be comparable to that of Earth, with amplitudes in the order of $10^{-9} \mathrm{~m} \mathrm{~s}^{-2} \mathrm{~Hz}^{-1 / 2}$ (Kobayashi \& Nishida 1998). According to Panning et al. (2017a), the detection of normal modes of this amplitude will be likely during nighttime measurements, when the external noise environment is low.

It remains to be resolved, whether the processing scheme applied here, will be applicable to and lead to results for the Martian data. Even the processing applied to different data sets from Earth is non-uniform and varies significantly. Fichtner et al. (2017) concluded that for each data set a suitable processing scheme needs to be developed through trial and error, which would also include the Martian data set. The answer to this issue will come, once data transmission from the SEIS instrument has started. Nevertheless, the processing scheme developed here is suitable for stations with varying subsurface structures and noise environments and might therefore also be applicable to the Martian data.

\section{Outlook}

Single-station correlations of seismic noise are a promising tool to extract information about the crustal thickness below the station. The developed method works well for the data set used throughout this work. However, several improvements to the method can be made before Mars data become available, in order to enhance the accuracy and precision of the results. Throughout this work, prior information was included in the form of target windows. Instead, prior information could be incorporated probabilistically by using a Bayesian approach. In addition, instead of looking at the vertical and horizontal correlations separately, a combined analysis could help with the interpretation of the results. One of the main pitfalls of the method is the assumption of vertical incidence. It was found throughout this work that this assumption is not always justified. One possibility would be to consider seismic events with known epicentral distances instead of seismic noise. If a velocity model is known, information on the incidence angle would be available, making 
it possible to obtain the vertical incidence reflection response and as a result the corrected depths.

Other single-station methods can also be used to determined the crustal thickness, such as, for example, receiver functions (Zhu \& Kanamori 2000) or measurements of surface wave dispersion. Receiver functions are widely used on Earth and have also been successfully applied to the Moon (Vinnik et al. 2001). The dispersion of surface waves can be used to extract information on subsurface structure from the relation between group velocity and frequency (Dehant et al. 2012). A combined analysis of the different methods, especially the receiver functions and autocorrelations, for example in the form of a joint inversion, can help in the interpretation of results. A joint inversion could decrease the need for prior information and improve the crustal thickness estimates, as well as help in the identification of shallower or deeper layers. In addition, receiver functions can provide $v_{\mathrm{P}} / v_{\mathrm{S}}$-ratios, if the location of the source and an average crustal $P$ - wave velocity are known. Comparing the receiver function results with the $v_{\mathrm{P}} / v_{\mathrm{S}}$-ratios obtained from the horizontal component correlations, the requirements and obtained values can be constrained and verified.

Throughout this work, the Moho is considered as the base of the crustal reflectivity. To understand the influence of a gradual velocity increase, or a Moho with topography, analysing synthetic seismograms from a known structure could help to understand and predict the influence on the waveforms of the correlations. A possible seismic wavefield could be obtained by using a temporarily and spatially random distribution of point sources with random characteristics (e.g. Gorbatov et al. 2013). Additionally, in order to estimate the applicability of the developed method to Mars, the method could be tested on synthetic seismograms for different models of Martian structure. From this, possible modifications to the processing could be derived, although it remains to be seen which processing is really suitable once the Martian data is available.

Proposals for futures lander missions to icy ocean worlds, such as Jupiter's moon Europa (Hand et al. 2017, Panning et al. 2017b, Stähler et al. 2017) and Saturn's moon Titan (Turtle et al. 2017), include seismometers to determine the thickness of the ice layer and underlying ocean. This provides the opportunity to further adapt the method to incorporate a liquid layer and a resulting wavefield different from what is seen on Earth. 



\section{Conclusions}

Wit beyond measure is man's greatest treasure.

J. K. Rowling

Autocorrelations and single-station cross-correlations of seismic noise can be used to extract the thickness of the crust and other subsurface layers below that station. This work has focused on the development of a processing scheme including high pass filtering, spectral smoothing, phase auto- and cross-correlation, and time-frequency domain phase weighted stacking, for both vertical and horizontal component seismic data. By incorporating prior information, the reflectivity change related to the Moho can be identified and determined automatically in the vertical component autocorrelations. The horizontal component correlations require manual identification of those reflectivity changes that are consistent across all correlations, leading to a range of lag times as a result. The obtained lag times are converted to depth. The determined Moho depths agree well with estimates obtained from other studies and are consistent across the three components. The largest average depth difference to results obtained in other studies is $2.4 \pm 3.1 \mathrm{~km}$. The method is applicable to different types of seismometers (short-period and broad-band) and does not require strong energy sources like earthquakes. The resulting autocorrelations are spatially stable and converge to a stable result after $\sim 6$ months of data for the vertical component and $\sim 8.5$ months of data for the horizontal component autocorrelations. The horizontal component cross-correlations require more time ( $\sim 17$ months) to reach stability.

The combined analysis of horizontal and component data can be used to infer information about the incidence angle of the seismic waves. For a few stations the converted phases can be observed in cross-correlations of the horizontal with vertical components. In addition, reflectivity changes associated with $P$-waves on horizontal component correlations and $S$-waves on vertical component correlations can be detected, indicating nonvertical incidence. Furthermore, $v_{\mathrm{P}} / v_{\mathrm{S}}$-ratios can be obtained from the determined lag times of reflectivity change in the vertical and horizontal component correlations. However, the $v_{\mathrm{P}} / v_{\mathrm{S}}$-ratios show great variability and thus needed to be treated with caution. The horizontal component autocorrelations can additionally be used to detect local crustal anisotropy.

Overall, single-station seismic noise correlations are a promising tool to extract information about the subsurface. They are especially valuable when the amount of available 
data is restricted, the presence of strong energy sources is limited, and station coverage is low. All these aspects are often conditions for seismic stations in remote areas, for example, on other planetary bodies. Another advantage of the method is the reduced need for three-component data. It is possible to extract the crustal thickness from vertical component data only, although three-component data is beneficial. With respect to the upcoming InSight mission to Mars, the method provides an additional means to extract the crustal thickness, thereby contributing to the fulfilment of the mission goals and helping in our understanding of the formation of terrestrial planets. 


\section{Bibliography}

Anderson, D. L., Miller, W. F., Latham, G. V., Nakamura, Y., Toksöz, M. N., Dainty, A. M., Duennebier, F. K., Lazarewicz, A. R., Kovach, R. L., \& D Knight, T. C., 1977. Seismology on Mars, J. geophys. Res., 82(28), 4524-4546.

Artemieva, I. M. \& Meissner, R., 2012. Crustal thickness controlled by plate tectonics: A review of crust-mantle interaction processes illustrated by European examples, Tectonophysics, 530-531, 18-49.

Aster, R. C., McNamara, D. E., \& Bromirski, P. D., 2008. Multidecadal climate-induced variability in microseisms, Seismol. Res. Lett., 79(2), 194-202.

Babuška, V. \& Plomerová, J., 2013. Boundaries of mantle-lithosphere domains in the Bohemian Massif as extinct exhumation channels for high-pressure rocks, Gondwana Res., 23, 973-987.

Banerdt, W. B., Smrekar, S., Hurst, K., Lognonné, P., Spohn, T., Asmar, S., Banfield, D., Boschi, L., Christensen, U., Dehant, V., Folkner, W., Giardini, D., Goetz, W., Golombek, M., Grott, M., Hudson, T., Johnson, C., Kargl, G., Kobayashi, N., Maki, J., Mimoun, D., Mocquet, A., \& Morgan, P., 2013. InSight: A discovery mission to explore the interior of Mars, in 44th Lunar and Planetary Science Conference, The Woodlands, Texas, p. 1915, Lunar and Planetary Institute, Houston.

Barlow, N. G., 2008. Mars - An introduction to its interior, surface and atmosphere, Cambridge University Press, Cambridge, 8th edn.

Becker, G. \& Knapmeyer-Endrun, B., 2018. Crustal thickness across the Trans-European Suture Zone from ambient noise autocorrelations, Geophys. J. Int., 212, 1237-1254.

Bogdanova, S., Gorbatschev, R., Grad, M., Janik, T., Guterch, A., Kozlovskaya, E., Motuza, G., Skridlaite, G., Starostenko, V., Taran, L., \& EUROBRIDGE \& POLONAISE Working Groups, 2006. EUROBRIDGE: new insight into the geodynamic evolution of the East European Craton, in European Lithosphere Dynamics, vol. 32, pp. 599-625, eds Gee, D. G. \& Stephenson, R. A., Geological Society of London.

Bokelmann, G. H. R. \& Baisch, S., 1999. Nature of narrow-band signals at $2.083 \mathrm{~Hz}$, Bull. seism. Soc. Am., 89(1), 156-164.

Bonnefoy-Claudet, S., Cotton, F., \& Bard, P.-Y., 2006. The nature of noise wavefield and its applications for site effects studies. A literature review, Earth-Sci. Rev., 79, 205-227. 
Brun, J. P., Gutscher, M.-A., \& DEKORP-ECORS Teams, 1992. Deep crustal structure of the Rhine Graben from DEKORP-ECORS seismic reflection data: a summary, Tectonophysics, 208(208), 139-147.

Campillo, M. \& Paul, A., 2003. Long-range correlations in the diffuse seismic coda, Science, 299, 547-549.

Campillo, M. \& Roux, P., 2015. Crust and lithospheric structure - Seismic imaging and monitoring with ambient noise correlations, in Treatise on Geophysics, chap. 1.12, pp. 391-417, ed. Schubert, G., Elsevier, Oxford, 2nd edn.

Chen, Y.-N., Gung, Y., Chiao, L.-Y., \& Rhie, J., 2017. Intrinsic noise level of noise cross-correlation functions and its implication to source population of ambient noises, Geophys. J. Int., 209, 141-151.

Claerbout, J. F., 1968. Synthesis of a layered medium from its acoustic transmission response, Geophysics, 33(2), 264-269.

Cristiano, L., Meier, T., Krüger, F., Keers, H., \& Weidle, C., 2016. Teleseismic P-wave polarization analysis at the Gräfenberg array, Geophys. J. Int., 207, 1456-1471.

Curtis, A., Gerstoft, P., Sato, H., Snieder, R., \& Wapenaar, K., 2006. Seismic interferometry - turning noise into signal, Leading Edge, 25(11), 1082-1096.

De Plaen, R. S. M., Lecocq, T., Caudron, C., Ferrazzini, V., \& Francis, O., 2016. Singlestation monitoring of volcanoes using seismic ambient noise, Geophys. Res. Lett., 43 , 8511-8518.

Deen, M., Wielandt, E., Stutzmann, E., Crawford, W., Barruol, G., \& Sigloch, K., 2017. First observation of the Earth's permanent free oscillations on ocean bottom seismometers, Geophys. Res. Lett., 44, 10988-10996.

Dehant, V., Banerdt, B., Lognonné, P., Grott, M., Asmar, S., Biele, J., Breuer, D., Forget, F., Jaumann, R., Johnson, C., Knapmeyer, M., Langlais, B., Le Feuvre, M., Mimoun, D., Mocquet, A., Read, P., Rivoldini, A., Romberg, O., Schubert, G., Smrekar, S., Spohn, T., Tortora, P., Ulamec, S., \& Vennerstrom, S., 2012. Future Mars geophysical observatories for understanding its internal structure, rotation, and evolution, Planet. Space Sci., 68, 123-145.

Delage, P., Karakostas, F., Dhemaied, A., Belmokhtar, M., Lognonné, P., Golombek, M., De Laure, E., Hurst, K., Dupla, J. C., Kedar, S., Cui, Y. J., \& Banerdt, B., 2017. An investigation of the mechanical properties of some Martian regolith simulants with respect to the surface properties at the InSight Mission landing site, Space Sci. Rev., 211, 191-213.

Draganov, D. S., Wapenaar, K., Mulder, W., Singer, J., \& Verdel, A., 2007. Retrieval of reflections from seismic background-noise measurements, Geophys. Res. Lett., 34, L04305. 
Duvall Jr., T. L., Jefferies, S. M., Harvey, J. W., \& Pomerantz, M. A., 1993. Time-distance helioseismology, Nature, 362, 430-432.

Fichtner, A., Ermert, L., \& Gokhberg, A., 2017. Seismic noise correlation on heterogeneous supercomputers, Seismol. Res. Lett., 88(4), 1141-1145.

Folkner, W. M., Yoder, C. F., Yuan, D. N., Standish, E. M., \& Preston, R. A., 1997. Interior structure and seasonal mass redistribution of Mars from radio tracking of Mars Pathfinder, Science, 278(5344), 1749-1752.

Folkner, W. M., Asmar, S. W., Dehant, V., \& Warwick, R. W., 2012. The Rotation and Interior Structure Experiment (RISE) for the InSight mission to Mars, in 43rd Lunar and Planetary Science Conference, The Woodlands, Texas, p. 1721, Lunar and Planetary Institute, Houston.

Fowler, C., 2005. The solid Earth: An introduction to global geophysics, Cambridge University Press, Cambridge, 2nd edn.

Geissler, W. H., Kind, R., \& Yuan, X., 2008. Upper mantle and lithospheric heterogeneities in central and eastern Europe as observed by teleseismic receiver functions, Geophys. J. Int., 174, 351-376.

Glahn, A. \& Granet, M., 1992. 3-D structure of the lithosphere beneath the southern Rhine Graben area, Tectonophysics, 208, 149-158.

Golombek, M., Kipp, D., Warner, N., Daubar, I. J., Fergason, R., Kirk, R. L., Beyer, R., Huertas, A., Piqueux, S., Putzig, N. E., Campbell, B. A., Morgan, G. A., Charalambous, C., Pike, W. T., Gwinner, K., Calef, F., Kass, D., Mischna, M., Ashley, J., Bloom, C., Wigton, N., Hare, T., Schwartz, C., Gengl, H., Redmond, L., Trautman, M., Sweeney, J., Grima, C., Smith, I. B., Sklyanskiy, E., Lisano, M., Benardini, J., Smrekar, S., Lognonné, P., \& Banerdt, W. B., 2017. Selection of the InSight landing site, Space Sci. Rev., 211, 5-95.

Golombek, M. P., 2002. A revision of Mars seismicity from surface faulting, in Lunar and Planetary Science Conference XXXIII, The Woodlands, Texas, p. 1244, Lunar and Planetary Institute, Houston.

Golombek, M. P., Banerdt, W. B., Tanaka, K. L., \& Tralli, D. M., 1992. A Prediction of Mars seismicity from surface faulting, Science, 258(5084), 979-981.

Goossens, S., Sabaka, T. J., Genova, A., Mazarico, E., Nicholas, J. B., \& Neumann, G. A., 2017. Evidence for a low bulk crustal density for Mars from gravity and topography, Geophys. Res. Lett., 44, 7686-7694.

Gorbatov, A., Saygin, E., \& Kennett, B. L. N., 2013. Crustal properties from seismic station autocorrelograms, Geophys. J. Int., 192, 861-870.

Grad, M. \& Tiira, T., 2012. Moho depth of the European Plate from teleseismic receiver functions, J. Seismol., 16, 95-105. 
Grad, M., Keller, G. R., Thybo, H., Guterch, A., \& POLONAISE Working Group, 2002. Lower lithospheric structure beneath the Trans-European Suture Zone from POLONAISE'97 seismic profiles, Tectonophysics, 360, 153-168.

Grad, M., Jensen, S. L., Keller, G. R., Guterch, A., Thybo, H., Janik, T., Tiira, T., Yliniemi, J., Luosto, U., Motuza, G., Nasedkin, V., Czuba, W., Gaczyński, E., Środa, P., Miller, K. C., Wilde-Piórko, M., Komminaho, K., Jacyna, J., \& Korabliova, L., 2003. Crustal structure of the Trans-European suture zone region along POLONAISE'97 seismic profile P4, J. geophys. Res., 108(B11), 2541.

Grad, M., Tiira, T., \& ESC Working Group, 2009. The Moho depth map of the European Plate, Geophys. J. Int., 176, 279-292.

Grad, M., Polkowski, M., \& Ostaficzuk, S. R., 2016. High-resolution 3D seismic model of the crustal and uppermost mantle structure in Poland, Tectonophysics, 666, 188-210.

Grotzinger, J. P., Crisp, J., Vasavada, A. R., Anderson, R. C., Baker, C. J., Barry, R., Blake, D. F., Conrad, P., Edgett, K. S., Ferdowski, B., Gellert, R., Gilbert, J. B., Golombek, M., Gómez-Elvira, J., Hassler, D. M., Jandura, L., Litvak, M., Mahaffy, P., Maki, J., Meyer, M., Malin, M. C., Mitrofanov, I., Simmonds, J. J., Vaniman, D., Welch, R. V., \& Wiens, R. C., 2012. Mars Science Laboratory mission and science investigation, Space Sci. Rev., 170, 5-56.

Guterch, A., Grad, M., Thybo, H., Keller, G. R., \& the POLONAISE Working Group, 1999. POLONAISE '97 - an international seismic experiment between Precambrian and Variscan Europe in Poland, Tectonophysics, 314, 101-121.

Hand, K. P., Murray, A. E., Garvin, J. B., Brinckerhoff, W. B., Christner, B. C., Edgett, K. S., Ehlmann, B. L., German, C. R., Hayes, A. G., Hoehler, T. M., Horst, S. M., Lunine, J. I., Nealson, K. H., Paranicas, C., Schmidt, B. E., Smith, D. E., Rhoden, A. R., Russell, M. J., Templeton, A. S., Willis, P. A., Yingst, R. A., Phillips, C. B., Cable, M. L., Craft, K. L., Hofmann, A. E., Nordheim, T. A., Pappalardo, R. P., \& Project Engineering Team, 2017. Report of the Europa lander science definiton team, Tech. rep., NASA.

Haned, A., Stutzmann, E., Schimmel, M., Kiselev, S., Davaille, A., \& Yelles-Chaouche, A., 2016. Global tomography using seismic hum, Geophys. J. Int., 204, 1222-1236.

Harjes, H.-P., 1990. Design and siting of a new regional array in Central Europe, Bull. seism. Soc. Am., 80(6), 1801-1817.

Hartmann, W. K., 2005. Martian cratering 8: Isochron refinement and the chronology of Mars, Icarus, 174, 294-320.

Hasselmann, K., 1963. A statistical analysis of the generation of microseisms, Rev. Geophys., 1(2), 177-210.

Heath, B. A., Hooft, E. E. E., \& Toomey, D. R., 2018. Autocorrelation of the seismic wavefield at Newberry volcano: Reflections from the magmatic and geothermal systems, Geophys. Res. Lett., 45, 2311-2318. 
Heidbach, O., Tingay, M., Barth, A., Reinecker, J., Kurfeß, D., \& Müller, B., 2010. Global crustal stress pattern based on the World Stress Map database release 2008, Tectonophysics, 482, 3-15.

Helffrich, G., 2017. Mars core structure-concise review and anticipated insights from InSight, Prog. Earth planet. Sci., 4(1), 24.

Hobiger, M., Wegler, U., Shiomi, K., \& Nakahara, H., 2014. Single-station crosscorrelation analysis of ambient seismic noise: Application to stations in the surroundings of the 2008 Iwate-Miyagi Nairiku earthquake, Geophys. J. Int., 198, 90-109.

Holstein-Rathlou, C., Gunnlaugsson, H. P., Merrison, J. P., Bean, K. M., Cantor, B. A., Davis, J. A., Davy, R., Drake, N. B., Ellehoj, M. D., Goetz, W., Hviid, S. F., Lange, C. F., Larsen, S. E., Lemmon, M. T., Madsen, M. B., Malin, M., Moores, J. E., Nørnberg, P., Smith, P., Tamppari, L. K., \& Taylor, P. A., 2010. Winds at the Phoenix landing site, J. geophys. Res., 115, E00E18.

Hrubcová, P. \& Geissler, W. H., 2009. The crust-mantle transition and the Moho beneath the Vogtland/West Bohemian region in the light of different seismic methods, Stud. Geophys. Geod., 53, 275-294.

Ito, Y. \& Shiomi, K., 2012. Seismic scatterers within subducting slab revealed from ambient noise autocorrelation, Geophys. Res. Lett., 39, L19303.

Ito, Y., Shiomi, K., Nakajima, J., \& Hino, R., 2012. Autocorrelation analysis of ambient noise in northeastern Japan subduction zone, Tectonophysics, 572-573, 38-46.

Iyer, H. M. \& Healy, J. H., 1972. Evidence for the existence of locally-generated body waves in the short-period noise at the large aperture seismic array, Montana, Bull. seism. Soc. Am., 62(1), 13-29.

Karousová, H., Plomerová, J., \& Babuška, V., 2012. Three-dimensional velocity model of the crust of the Bohemian Massif and its effects on seismic tomography of the upper mantle, Stud. Geophys. Geod., 56, 249-267.

Kaula, W. M., 1979. The moment of inertia of Mars, Geophys. Res. Lett., 6(3), 194-196.

Kedar, S., Andrade, J., Banerdt, B., Delage, P., Golombek, M., Grott, M., Hudson, T., Kiely, A., Knapmeyer, M., Knapmeyer-Endrun, B., Krause, C., Kawamura, T., Lognonne, P., Pike, T., Ruan, Y., Spohn, T., Teanby, N., Tromp, J., \& Wookey, J., 2017. Analysis of regolith properties using seismic signals generated by InSight's HP3 penetrator, Space Sci. Rev., 211, 315-337.

Kenda, B., Lognonné, P., Spiga, A., Kawamura, T., Kedar, S., Banerdt, W. B., Lorenz, R., Banfield, D., \& Golombek, M., 2017. Modeling of ground deformation and shallow surface waves generated by Martian dust devils and perspectives for near-surface structure inversion, Space Sci. Rev., 211, 501-524.

Kennett, B. L. N., 2015. Lithosphere-asthenosphere P-wave reflectivity across Australia, Earth planet. Sci. Lett., 431, 225-235. 
Kennett, B. L. N. \& Engdahl, E. R., 1991. Travel times for global earthquake location and phase association, Geophys. J. Int, 105, 429-465.

Kennett, B. L. N., Saygin, E., \& Salmon, M., 2015. Stacking autocorrelograms to map Moho depth with high spatial resolution in southeastern Australia, Geophys. Res. Lett., 42(18), 7490-7497.

Klein, H. P., Lederberg, J., Rich, A., Horowitz, N. H., Oyama, V. I., \& Levin, G. V., 1976. The Viking Mission search for life on Mars, Nature, 262, 24-27.

Knapmeyer, M., Oberst, J., Hauber, E., Wählisch, M., Deuchler, C., \& Wagner, R., 2006. Working models for spatial distribution and level of Mars' seismicity, J. geophys. Res., 111, E11006.

Knapmeyer-Endrun, B., Krüger, F., \& The PASSEQ Working Group, 2014. Moho depth across the Trans-European Suture Zone from P- and S-receiver functions, Geophys. $J$. Int., 197, 1048-1075.

Knapmeyer-Endrun, B., Golombek, M. P., \& Ohrnberger, M., 2017a. Rayleigh wave ellipticity modeling and inversion for shallow structure at the proposed InSight landing site in Elysium Planitia, Mars, Space Sci. Rev., 211, 339-382.

Knapmeyer-Endrun, B., Krüger, F., \& Geissler, W. H., 2017b. Upper mantle structure across the Trans-European Suture Zone imaged by S-receiver functions, Earth planet. Sci. Lett., 458, 429-441.

Kobayashi, N. \& Nishida, K., 1998. Continuous excitation of planetary free oscillations by atmospheric disturbances, Nature, 395, 357-360.

Konopliv, A. S., Park, R. S., \& Folkner, W. M., 2016. An improved JPL Mars gravity field and orientation from Mars orbiter and lander tracking data, Icarus, 274, 253-260.

Lamarche, J. \& Scheck-Wenderoth, M., 2005. 3D structural model of the Polish Basin, Tectonophysics, 397, 73-91.

Larose, E., Khan, A., Nakamura, Y., \& Campillo, M., 2005. Lunar subsurface investigated from correlation of seismic noise, Geophys. Res. Lett., 32, L16201.

Lin, F. C., Moschetti, M. P., \& Ritzwoller, M. H., 2008. Surface wave tomography of the western United States from ambient seismic noise: Rayleigh and Love wave phase velocity maps, Geophys. J. Int., 173, 281-298.

Lin, F. C., Tsai, V. C., Schmandt, B., Duputel, Z., \& Zhan, Z., 2013. Extracting seismic core phases with array interferometry, Geophys. Res. Lett., 40, 1049-1053.

Lognonné, P. \& Mosser, B., 1993. Planetary Seismology, Surv. Geophys., 14, 239-302.

Lognonné, P., Banerdt, W. B., Weber, R. C., Giardini, D., Pike, W. T., Christensen, U., Mimoun, D., Clinton, J., Dehant, V., Garcia, R., John-Son, C. L., Kobayashi, N., Knapmeyer-Endrun, B., Mocquet, A., Panning, M., Smrekar, S., Tromp, J., Wieczorek, 
M., Beucler, E., Drilleau, M., Kawamura, T., Kedar, S., Murdoch, N., Laudet, P., \& InSight/SEIS Team, 2015. Science goals of the SEIS, the InSight seismometer package, in 46th Lunar and Planetary Science Conference, The Woodlands, Texas, p. 2272, Lunar and Planetary Institute, Houston.

Lomb, N. R., 1976. Least-squares frequency analysis of unequally spaced data, Astrophys. Space Sci., 39, 447-462.

Lombardi, D., Braunmiller, J., Kissling, E., \& Giardini, D., 2008. Moho depth and Poisson's ratio in the Western-Central Alps from receiver functions, Geophys. J. Int., 173, 249-264.

Longuet-Higgins, M. S., 1950. A theory of the origin of microseisms, Phil. Trans. Royal Soc., 243(857), 1-35.

Maeda, T., Ohara, K., \& Yukutake, Y., 2010. Seismic velocity decrease and recovery related to earthquake swarms in a geothermal area, Earth Planets Space, 62, 685-691.

Majdański, M., 2012. The structure of the crust in TESZ area by kriging interpolation, Act. Geophys., 60(1), 59-75.

Matte, P., Maluski, H., Rajlich, P., \& Franke, W., 1990. Terrane boundaries in the Bohemian Massif: Result of large-scale Variscan shearing, Tectonophysics, 177, 151-170.

Meier, T., Soomro, R. A., Viereck, L., Lebedev, S., Behrmann, J. H., Weidle, C., Cristiano, L., \& Hanemann, R., 2016. Mesozoic and Cenozoic evolution of the Central European lithosphere, Tectonophysics, 692, 58-73.

Mimoun, D., Murdoch, N., Lognonné, P., Hurst, K., Pike, W. T., Hurley, J., Nébut, T., Banerdt, W. B., \& SEIS Team, 2017. The noise model of the SEIS seismometer of the InSight Mission to Mars, Space Sci. Rev., 211, 383-428.

Molinari, I. \& Morelli, A., 2011. EPcrust: A reference crustal model for the European Plate, Geophys. J. Int., 182, 352-364.

Murdoch, N., Kenda, B., Kawamura, T., Spiga, A., Lognonné, P., Mimoun, D., \& Banerdt, W. B., 2017a. Estimations of the seismic pressure noise on Mars determined from large eddy simulations and demonstration of pressure decorrelation techniques for the Insight Mission, Space Sci. Rev., 211, 457-483.

Murdoch, N., Mimoun, D., Garcia, R. F., Rapin, W., Kawamura, T., Lognonné, P., Banfield, D., \& Banerdt, W. B., 2017b. Evaluating the wind-induced mechanical noise on the InSight seismometers, Space Sci. Rev., 211, 429-455.

Nakamura, Y. \& Anderson, D. L., 1979. Martian wind activity detected by a seismometer at Viking Lander 2 site, Geophys. Res. Lett., 6(6), 499-502.

NASA Mars Exploration Program \& Jet Propulsion Laboratory, 2014. NASA Science Mars Exploration Program, Last accessed: 2018-04-16, https://mars .nasa.gov/. 
Neukum, G., Jaumann, R., Hoffmann, H., Hauber, E., Head, J. W., Basilevsky, A. T., Ivanov, B. A., Werner, S. C., Van Gasselt, S., Murray, J. B., McCord, T., \& The HRSC Co-Investigator Team, 2004. Recent and episodic volcanic and glacial activity on Mars revealed by the High Resolution Stereo Camera, Nature, 432, 971-979.

Neumann, G. A., Zuber, M. T., Wieczorek, M. A., McGovern, P. J., Lemoine, F. G., \& Smith, D. E., 2004. Crustal structure of Mars from gravity and topography, J. geophys. Res., 109, E08002.

Nielsen, L., Thybo, H., \& Egorkin, A. V., 2002. Implications of seismic scattering below the $8^{\circ}$ discontinuity along PNE profile Kraton, Tectonophysics, 358, 135-150.

Nishida, K., 2013. Global propagation of body waves revealed by cross-correlation analysis of seismic hum, Geophys. Res. Lett., 40, 1691-1696.

Nishitsuji, Y., Rowe, C. A., Wapenaar, K., \& Draganov, D., 2016. Reflection imaging of the Moon's interior using deep-moonquake seismic interferometry, J. geophys. Res., 121, 695-713.

Ohmi, S., Hirahara, K., Wada, H., \& Ito, K., 2008. Temporal variations of crustal structure in the source region of the 2007 Noto Hanto earthquake, central Japan, with passive image interferometry, Earth Planets Space, 60, 1069-1074.

Oren, C. \& Nowack, R. L., 2017. Seismic body-wave interferometry using noise autocorrelations for crustal structure, Geophys. J. Int., 208, 321-332.

Panning, M. P., Beucler, É., Drilleau, M., Mocquet, A., Lognonné, P., \& Banerdt, W. B., 2015. Verifying single-station seismic approaches using Earth-based data: Preparation for data return from the InSight mission to Mars, Icarus, 248, 230-242.

Panning, M. P., Lognonné, P., Bruce Banerdt, W., Garcia, R., Golombek, M., Kedar, S., Knapmeyer-Endrun, B., Mocquet, A., Teanby, N. A., Tromp, J., Weber, R., Beucler, E., Blanchette-Guertin, J. F., Bozdağ, E., Drilleau, M., Gudkova, T., Hempel, S., Khan, A., Lekić, V., Murdoch, N., Plesa, A. C., Rivoldini, A., Schmerr, N., Ruan, Y., Verhoeven, O., Gao, C., Christensen, U., Clinton, J., Dehant, V., Giardini, D., Mimoun, D., Thomas Pike, W., Smrekar, S., Wieczorek, M., Knapmeyer, M., \& Wookey, J., 2017a. Planned products of the Mars structure service for the InSight Mission to Mars, Space Sci. Rev., 211, 611-650.

Panning, M. P., Stähler, S. C., Huang, H.-H., Vance, S. D., Kedar, S., Tsai, V. C., Pike, W. T., \& Lorenz, R. D., 2017b. Expected seismicity and the seismic noise environment of Europa, J. geophys. Res., 123, 163-179.

Pasyanos, M. E., 2010. Lithospheric thickness modeled from long-period surface wave dispersion, Tectonophysics, 481, 38-50.

Pasyanos, M. E., Masters, T. G., Laske, G., \& Ma, Z., 2014. LITHO1.0: An updated crust and lithospheric model of the Earth, J. geophys. Res., 119, 2153-2173. 
Phạm, T. S. \& Tkalčić, H., 2017. On the feasibility and use of teleseismic P wave coda autocorrelation for mapping shallow seismic discontinuities, J. geophys. Res., 122, 37763791.

Phillips, R. J., 1991. Expected rates of Marsquakes, in Scientific rationale and requirements for a global seismic network on Mars, LPI Technical Report, 91-02, Lunar and Planetary Institute, Houston.

Plesa, A. C., Grott, M., Tosi, N., Breuer, D., Spohn, T., \& Wieczorek, M. A., 2016. How large are present-day heat flux variations across the surface of Mars?, J. geophys. Res., 121, 2386-2403.

Plesa, A.-C., Knapmeyer, M., Golombek, M. P., Breuer, D., Grott, M., Kawamura, T., Lognonné, P., Tosi, N., \& Weber, R. C., 2018. Present-day Mars' seismicity predicted from 3-D thermal evolution models of interior dynamics, Geophys. Res. Lett., 45, 2580-2589.

Poli, P., Campillo, M., Pedersen, H., \& LAPNET Working Group, 2012a. Body-wave imaging of Earth' s mantle discontinuities from ambient seismic noise, Science, 338, $1063-1065$.

Poli, P., Pedersen, H. A., \& Campillo, M., 2012b. Emergence of body waves from crosscorrelation of short period seismic noise, Geophys. J. Int., 188, 549-558.

Rabbel, W. \& Mooney, W. D., 1996. Seismic anisotropy of the crystalline crust: What does it tell us?, Terra Nova, 8, 16-21.

Roberts, G. P., Matthews, B., Bristow, C., Guerrieri, L., \& Vetterlein, J., 2012. Possible evidence of paleomarsquakes from fallen boulder populations, Cerberus Fossae, Mars, J. geophys. Res., 117, E02009.

Roux, P., Sabra, K. G., Gerstoft, P., Kuperman, W. A., \& Fehler, M. C., 2005. P-waves from cross-correlation of seismic noise, Geophys. Res. Lett., 23, L19303.

Ruigrok, E., Campman, X., \& Wapenaar, K., 2011. Extraction of P-wave reflections from microseisms, C. R. Geosci., 343, 512-525.

Růžek, B., Vavryčuk, V., Hrubcová, P., Zedník, J., \& the CELEBRATION Working Group, 2000. Crustal anisotropy in the Bohemian Massif, Czech Republic: Observations based on Central European Lithospheric Experiment Based on Refraction (CELEBRATION) 2000, J. geophys. Res, 108(B8), 2392.

Saygin, E., Cummins, P. R., \& Lumley, D., 2017. Retrieval of the P wave reflectivity response from autocorrelation of seismic noise: Jakarta Basin, Indonesia, Geophys. Res. Lett., 44, 792-799.

Scargle, J. D., 1982. Studies in astronomical time series analysis. II - Statistical aspects of spectral analysis of unevenly spaced data, ApJ, 263, 835-853. 
Scheck-Wenderoth, M. \& Lamarche, J., 2005. Crustal memory and basin evolution in the Central European Basin System - new insights from a 3D structural model, Tectonophysics, 397, 143-165.

Schimmel, M., 1999. Phase cross-correlations: Design, comparisons, and applications, Bull. seism. Soc. Am., 89(5), 1366-1378.

Schimmel, M. \& Gallart, J., 2007. Frequency-dependent phase coherence for noise suppression in seismic array data, J. geophys. Res., 112, B04303.

Schimmel, M. \& Paulssen, H., 1997. Noise reduction and detection of weak, coherent signals through phase-weighted stacks, Geophys. J. Int., 130, 497-505.

Schimmel, M., Stutzmann, E., Ardhuin, F., \& Gallart, J., 2011a. Polarized Earth's ambient microseismic noise, Geochem. Geophys. Geosys., 12(7), Q07014.

Schimmel, M., Stutzmann, E., \& Gallart, J., 2011b. Using instantaneous phase coherence for signal extraction from ambient noise data at a local to a global scale, Geophys. J. Int., 184, 494-506.

Schulmann, K., Konopásek, J., Janoušek, V., Lexa, O., Lardeaux, J. M., Edel, J. B., Štípská, P., \& Ulrich, S., 2009. An Andean type Palaeozoic convergence in the Bohemian Massif, C. R. Geosci., 341, 266-286.

Schuster, G. T., 2009. Seismic Interferometry, Cambridge University Press, Cambridge.

Sens-Schönfelder, C. \& Larose, E., 2010. Lunar noise correlation, imaging and monitoring, Earthq. Sci., 23, 519-530.

Sens-Schönfelder, C. \& Wegler, U., 2006. Passive image interferometry and seasonal variations of seismic velocities at Merapi Volcano, Indonesia, Geophys. Res. Lett., 33, L21302.

Shapiro, N. M. \& Campillo, M., 2004. Emergence of broadband Rayleigh waves from correlations of the ambient seismic noise, Geophys. Res. Lett., 31, L07614.

Shapiro, N. M., Campillo, M., Stehly, L., \& Ritzwoller, M. H., 2005. High-resolution surface-wave tomography from ambient seismic noise, Science, 307(5715), 16151618.

Shearer, P., 2009. Introduction to Seismology, Cambridge University Press, Cambridge, 2nd edn.

Spohn, T., Grott, M., Knollenberg, J., Zoest, T. v., Kargl, G., Smrekar, S. E., Banerdt, W. B., Hudson, T. L., \& the HP3 Instrument Team, 2012. InSight: Measuring the Martian heat flow using the Heat Flow and Physical Properties Package (HP3), in 43rd Lunar Planetary Science Conference, The Woodlands, Texas, p. 1445, Lunar and Planetary Institute, Houston. 
Stähler, S. C., Panning, M. P., Vance, S. D., Lorenz, R., van Driel, M., Nissen-Meyer, T., \& Kedar, S., 2017. Seismic wave propagation in icy ocean worlds, J. geophys. Res., 123, 206-232.

Stehly, L., Fry, B., Campillo, M., Shapiro, N. M., Guilbert, J., Boschi, L., \& Giardini, D., 2009. Tomography of the Alpine region from observations of seismic ambient noise, Geophys. J. Int., 178, 338-350.

Stockwell, R. G., Mansinha, L., \& Lowe, R. P., 1996. Localization of the complex spectrum: The S transform, IEEE Trans. Signal Process., 44(4), 998-1001.

Suda, N., Nawa, K., \& Fukao, Y., 1998. Earth's background free oscillations, Science, 279, 2089-2091.

Sun, W. \& Kennett, B. L., 2016. Receiver structure from teleseisms: Autocorrelation and cross correlation, Geophys. Res. Lett., 43, 6234-6242.

Sun, W. \& Kennett, B. L. N., 2017. Mid-lithosphere discontinuities beneath the western and central North China Craton, Geophys. Res. Lett., 44, 1302-1310.

Tanimoto, T., Eitzel, M. V., \& Yano, T., 2008. The noise cross-correlation approach for Apollo 17 LSPE data: Diurnal change in seismic parameters in shallow lunar crust, $J$. geophys. Res., 113, E08011.

Taylor, F. W., 2010. The Scientific Exploration of Mars, Cambridge University Press, Cambridge.

Taylor, G., Rost, S., \& Houseman, G., 2016. Crustal imaging across the North Anatolian Fault Zone from the autocorrelation of ambient seismic noise, Geophys. Res. Lett., 43, 2502-2509.

Taylor, J., Teanby, N. A., \& Wookey, J., 2013. Estimates of seismic activity in the Cerberus Fossae region of Mars, J. geophys. Res., 118, 2570-2581.

Teanby, N. A., 2015. Predicted detection rates of regional-scale meteorite impacts on Mars with the InSight short-period seismometer, Icarus, 256, 49-62.

Teanby, N. A. \& Wookey, J., 2011. Seismic detection of meteorite impacts on Mars, Phys. Earth planet. Int., 186, 70-80.

Tibuleac, I. M. \& von Seggern, D., 2012. Crust-mantle boundary reflectors in Nevada from ambient seismic noise autocorrelations, Geophys. J. Int., 189, 493-500.

Turtle, E. P., Barnes, J. W., Trainer, M. G., Lorenz, R. D., Mackenzie, S. M., Hibbard, K. E., Adams, D., Bedini, P., Langelaan, J. W., Zacny, K., \& the Dragonfly Team, 2017. Dragonfly: Exploring Titan's prebiotic organic chemistry and habitability, in Lunar and Planetary Science Conference XLVIII, The Woodlands, Texas, p. 1958, Lunar and Planetary Institute, Houston. 
Van Hoolst, T., Dehant, V., Roosbeek, F., \& Lognonné, P., 2003. Tidally induced surface displacements, external potential variations, and gravity variations on Mars, Icarus, 161, 281-296.

Vetterlein, J. \& Roberts, G. P., 2010. Structural evolution of the Northern Cerberus Fossae graben system, Elysium Planitia, Mars, J. struct. Geol., 23, 394-406.

Vinnik, L., Chenet, H., Gagnepain-Beyneix, J., \& Lognonné, P., 2001. First seismic receiver functions on the Moon, Geophys. Res. Lett., 28(15), 3031-3034.

Vinnik, L. P., 1973. Sources of microseismic P waves, Pure appl. Geophys., 103(1), 282-289.

Wang, T., Song, X., \& Xia, H. H., 2015. Equatorial anisotropy in the inner part of Earth's inner core from autocorrelation of earthquake coda, Nature Geosci., 8, 224-227.

Wapenaar, K., 2003. Synthesis of an inhomogeneous medium from its acoustic transmission response, Geophysics, 68(5), 1756-1759.

Wapenaar, K. \& Fokkema, J., 2006. Green's function representations for seismic interferometry, Geophysics, 71(4), SI33-SI46.

Wapenaar, K., Thorbecke, J., \& Draganov, D., 2004. Relations between reflection and transmission responses of three-dimensional inhomogeneous media, Geophys. J. Int., 156, 179-194.

Wapenaar, K., Draganov, D., Snieder, R., Campman, X., \& Verdel, A., 2010. Tutorial on seismic interferometry: Part 1 - Basic principles and applications, Geophysics, 75(5), 75A195-75A209.

Warner, N. H., Golombek, M. P., Sweeney, J., Fergason, R., Kirk, R., \& Schwartz, C., 2017. Near surface stratigraphy and regolith production in southwestern Elysium Planitia, Mars: Implications for Hesperian-Amazonian terrains and the InSight lander mission, Space Sci. Rev., 211, 147-190.

Weaver, R. L. \& Lobkis, O. I., 2001. Ultrasonics without a source: Thermal fluctuation correlations at MHz frequencies, Phys. Rev. Lett., 87(13), 134301-1-134301-4.

Wegler, U. \& Sens-Schönfelder, C., 2007. Fault zone monitoring with passive image interferometry, Geophys. J. Int., 168, 1029-1033.

Wieczorek, M. A., 2015. Gravity and topography of the terrestrial planets, in Treatise on Geophysics, chap. 10.05, pp. 153-193, ed. Schubert, G., Elsevier, Oxford, 2nd edn.

Wieczorek, M. A. \& Zuber, M. T., 2004. Thickness of the Martian crust: Improved constraints from geoid-to-topography ratios, J. geophys. Res., 109, E01009.

Wiggins, R. A., 1972. The general linear inverse problem: Implication of surface waves and free oscillations for Earth structure, Rev. Geophys. Space Phys., 10(1), 251-285. 
Wilde-Piórko, M., Saul, J., \& Grad, M., 2005. Differences in the crustal and uppermost mantle structure of the Bohemian Massif from teleseismic receiver functions, Stud. Geophys. Geod., 49, 85-107.

Wilde-Piórko, M., Geissler, W. H., Plomerová, J., Knapmeyer-Endrun, B., Grad, M., Babuška, V., Brückl, E., Cyziene, J., Czuba, W., England, R., Gaczyński, E., Gazdova, R., Gregersen, S., Guterch, A., Hanka, W., Hegedûs, E., Heuer, B., Jedlička, P., Lazauskiene, J., Keller, G. R., Kind, R., Klinge, K., Kolinsky, P., Komminaho, K., Kozlovskaya, E., Krüger, F., Larsen, T., Majdański, M., Malek, J., Motuza, G., Novotný, O., Pietrasiak, R., Plenefisch, T., Růžek, B., Sliaupa, S., Środa, P., Świeczak, M., Tiira, T., Voss, P., \& Wiejacz, P., 2006. PASSEQ 2006-2008: Passive Seismic Experiment in Trans-European Suture Zone, Tech. rep., Deutsches GeoForschungsZentrum GFZ, doi:10.14470/2R383989, Seismic Network.

Wilde-Piórko, M., Geissler, W. H., Plomerová, J., Grad, M., Babuška, V., Brückl, E., Cyziene, J., Czuba, W., England, R., Gaczyński, E., Gazdova, R., Gregersen, S., Guterch, A., Hanka, W., Hegedûs, E., Heuer, B., Jedlička, P., Lazauskiene, J., Keller, G. R., Kind, R., Klinge, K., Kolinsky, P., Komminaho, K., Kozlovskaya, E., Krüger, F., Larsen, T., Majdański, M., Málek, J., Motuza, G., Novotný, O., Pietrasiak, R., Plenefisch, T., Růžek, B., Sliaupa, S., Środa, P., Świeczak, M., Tiira, T., Voss, P., \& Wiejacz, P., 2008. PASSEQ 2006-2008: Passive seismic experiment in trans-European suture zone, Stud. Geophys. Geod., 52, 439-448.

Wilde-Piórko, M., Świeczak, M., Grad, M., \& Majdański, M., 2010. Integrated seismic model of the crust and upper mantle of the Trans-European Suture zone between the Precambrian craton and Phanerozoic terranes in Central Europe, Tectonophysics, 481, $108-115$.

Wilde-Piórko, M., Grycuk, M., Polkowski, M., \& Grad, M., 2017. On the rotation of teleseismic seismograms based on the receiver function technique, J. Seismol., 21(4), 857-868.

Xia, H. H., Song, X., \& Wang, T., 2016. Extraction of triplicated PKP phases from noise correlations, Geophys. J. Int., 205, 499-508.

Zhan, Z., Ni, S., Helmberger, D. V., \& Clayton, R. W., 2010. Retrieval of Moho-reflected shear wave arrivals from ambient seismic noise, Geophys. J. Int., 182, 408-420.

Zhu, L. \& Kanamori, H., 2000. Moho depth variation in southern California from teleseismic receiver functions, J. geophys. Res., 105(B2), 2969-2980.

Ziegler, P. A., 1992. European Cenozoic rift system, Tectonophysics, 208, 91-111.

Zuber, M. T., Solomon, S. C., Phillips, R. J., Smith, D. E., Tyler, G. L., Aharonson, O., Balmino, G., Banerdt, W. B., Head, J. W., Johnson, C. L., Lemoine, F. G., McGovern, P. J., Neumann, G. A., Rowlands, D. D., \& Zhong, S., 2000. Internal structure and early thermal evolution of Mars from Mars Global Surveyor topography and gravity, Science, 287(5459), 1788-1793. 



\section{Acknowledgements}

First, I would like to thank Brigitte for giving me the opportunity to work on this project. Without her constant support and guidance, this work would not have been possible. I would like to thank Uli and Laurent for being part of my Thesis Advisory Committee, providing valuable advice and agreeing to read this thesis. Thank you also goes out to Walter Götz, Wolfram Kollatschny and Andreas Tilgner for agreeing to be on my thesis committee and to Frank Krüger for reading this thesis.

I would like to thank Sonja for her patience, help and support throughout the time of my PhD and the International Max Planck Research School for Solar System Science at the University of Göttingen for providing the framework of this work. I would like to thank the Geodynamo group for adopting me and patiently trying to explain the intricacies of magneto-hydrodynamics. Thank you to all the members of the institute, who helped and supported me throughout this PhD.

A big thank you goes out to all my friends and colleagues at the institute. Thank you for making this $\mathrm{PhD}$ a memorable time. I would especially like to thank all past and current members of office Fun01 for keeping me focused, motivated and entertained, for all their advice and help and for creating a comfortable working environment. Thank you to the coffee group for all the chocolate and for making the after lunch dip bearable. Thank you to the sports group for providing a healthy balance to the office work. Thank you very much to everyone who also made the life outside the institute fun and enjoyable.

A final important thank you goes out to my family for supporting me and for always having my back. Without them I would not be who and where I am today. 



\title{
Curriculum vitae
}

\author{
Gesa Karen Becker \\ Born 18.12.1989, in Langenhagen, Deutschland \\ Nationality: German
}

\section{Education}

2015-2018: PhD Student, Max Planck Institute for Solar System Research and Georg-August-Universität Göttingen, Germany

Thesis Title: "Crustal thickness from seismic noise correlations in preparation for the InSight mission to Mars"

Supervisors: Dr. B. Knapmeyer-Endrun, Prof. Dr. U. Christensen and

Prof. Dr. L. Gizon

2012-2015: Master of Science in Geosciences with specialization in Geophysics

Universität Potsdam, Germany

Title: "Application of several ground-penetrating radar systems in the vicinity of the Stern Hunting Lodge in Potsdam"

Supervisors: Dr. E. Lück and Prof. Dr. J. Tronicke

2009-2012: Bachelor of Science in Geosciences

Universität Potsdam, Germany

Title: "Tsunami hazard assessment of Porirua, New Zealand"

Supervisor: O. Korup, $\mathrm{PhD}$

\section{Publications}

Refereed Papers:

1. "Crustal thickness across the Trans-European Suture zone from ambient noise autocorrelations" - G. Becker, B. Knapmeyer-Endrun. 2018. Geophysical Journal International, 212, 1237-1254.

2. "Crustal thickness from horizontal component seismic noise auto- and cross-correlations for stations in Central and Eastern Europe" - G. Becker, B. Knapmeyer-Endrun. 2018. Geophysical Journal International, submitted. 